\title{
Minimal surfaces in pseudohermitian geometry
}

\author{
Jih-Hsin Cheng, Jenn-Fang Hwang, Andrea Malchiodi
}

AND PAUl YANG

\begin{abstract}
We consider surfaces immersed in three-dimensional pseudohermitian manifolds. We define the notion of (p-)mean curvature and of the associated (p-)minimal surfaces, extending some concepts previously given for the (flat) Heisenberg group. We interpret the p-mean curvature not only as the tangential sublaplacian of a defining function, but also as the curvature of a characteristic curve, and as a quantity in terms of calibration geometry.

As a differential equation, the p-minimal surface equation is degenerate (hyperbolic and elliptic). To analyze the singular set (i.e., the set where the (p-)area integrand vanishes), we formulate some extension theorems, which describe how the characteristic curves meet the singular set. This allows us to classify the entire solutions to this equation and to solve a Bernstein-type problem (for graphs over the $x y$-plane) in the Heisenberg group $H_{1}$. In $H_{1}$, identified with the Euclidean space $\mathbb{R}^{3}$, the $p$-minimal surfaces are classical ruled surfaces with the rulings generated by Legendrian lines. We also prove a uniqueness theorem for the Dirichlet problem under a condition on the size of the singular set in two dimensions, and generalize to higher dimensions without any size control condition.

We also show that there are no closed, connected, $C^{2}$ smoothly immersed constant p-mean curvature or p-minimal surfaces of genus greater than one in the standard $S^{3}$. This fact continues to hold when $S^{3}$ is replaced by a general pseudohermitian 3-manifold.
\end{abstract}

Mathematics Subject Classification (2000): 35L80 (primary); 35J70, 32V20, 53A10, 49Q10 (secondary).

\section{Introduction and statement of the results}

Minimal surfaces in a Riemannian manifold play an important role in the study of the topology and the geometry of the ambient manifold. For instance, the positive mass theorem originally was proved with the aid of minimal surface theory ([SY]). In order to study the mass in an analogous manner or to formulate a boundary value problem for prescribing the Webster scalar curvature 
on a domain with boundary in pseudohermitian geometry, we find it necessary to formulate a notion of mean curvature for a surface in a pseudohermitian manifold.

Let $\Sigma$ be a surface in a 3-dimensional pseudohermitian manifold $M$. Roughly speaking, $M$ is a manifold having a (contact) plane distribution on which an almost complex structure is defined (see the Appendix for some basic facts in pseudohermitian geometry). A singular point of $\Sigma$ is a point where the background (contact) plane coincides with the tangent plane of $\Sigma$. To every nonsingular point of $\Sigma$, we associate a vector field normal to $\Sigma$, called the Legendrian normal. If for example $\Sigma$ bounds some smooth set $\Omega$, then the p-area, in analogy with the Riemannian case, is obtained as the variation of the volume of $\Omega$ in the direction of the Legendrian normal (see formulas (2.4) and (2.5)).

The p-mean curvature $H$ of $\Sigma$ is in turn given by the first variation of the p-area. It is easy to see that $H$ equals the negative subdivergence of the Legendrian normal $e_{2}$. Suppose $\psi$ is a defining function of $\Sigma$ such that $e_{2}=\nabla_{b} \psi /\left|\nabla_{b} \psi\right|_{G}(G$ is the Levi metric. See the definition in Section 2). Then the p-mean curvature equation and the p-minimal surface equation $(H \equiv 0)$ read

$$
-\operatorname{div}_{b}\left(\nabla_{b} \psi /\left|\nabla_{b} \psi\right|_{G}\right)=H
$$

and

$$
\operatorname{div}_{b}\left(\nabla_{b} \psi /\left|\nabla_{b} \psi\right|_{G}\right)=0,
$$

(pMSE)

respectively.

Alternatively, having in mind the Gauss map, the mean curvature can be defined in terms of the covariant derivative (with respect to the pseudohermitian connection) along $\Sigma$ of the Legendrian tangent $e_{1}$ to $\Sigma$ (see (2.1)).

Of course the case of the Heisenberg group as a pseudohermitian manifold is one of the most important. Indeed it is the simplest model example, and represents a blow-up limit of general pseudo-hermitian manifolds. In the case of a smooth surface in the Heisenberg group, our definitions coincide with those given in [CDG, DGN], and [Pau]. In particular these notions, especially in the framework of geometric measure theory, have been used to study existence or regularity properties of minimizers for the relative perimeter or extremizers of isoperimetric inequalities (see, e.g., [DGN, GN, LM, LR], and [Pan]). The parea can also be identified with the 3-dimensional spherical Hausdorff measure of $\Sigma$ (see, e.g., [B, FSS]).

In this paper, we study the subject mainly from the viewpoint of partial differential equations and from that of differential geometry. Our basic results are the analysis of the singular set (see Section 3). As consequences, we can prove a Bernstein-type theorem (see Section 4 and Theorem A) and the nonexistence of closed hyperbolic p-minimal surfaces (see Section 7 and Theorem E). We also establish a comparison theorem (see Section 5) which is a substitute for the maximum principle and may become a useful tool in the subject. 
For a p-minimal graph $(x, y, u(x, y))$ in the Heisenberg group $H_{1}$, the above equation (pMSE) reduces to

$$
\left(u_{y}+x\right)^{2} u_{x x}-2\left(u_{y}+x\right)\left(u_{x}-y\right) u_{x y}+\left(u_{x}-y\right)^{2} u_{y y}=0
$$

by taking $\psi=z-u(x, y)$ on the nonsingular domain. This is a degenerate (hyperbolic and elliptic) partial differential equation. It is degenerate hyperbolic (on the nonsingular domain) having only one characteristic direction (note that a 2-dimensional hyperbolic equation has two characteristic directions [Jo]). We call the integral curves of this characteristic direction the characteristic curves. We show that the p-mean curvature is the line curvature of a characteristic curve. Therefore the characteristic curves of (pMGE) are straight lines. Moreover, the value of $u$ along a characteristic curve is determined in a simple way (see (2.22), (2.23)).

The analysis of the singular set is necessary to characterize the solutions. As long as the behavior of $H$ (consider (pMCE) for a graph in $H_{1}$ ) is not too bad (say, bounded), we show that the singular set consists of only isolated points and smooth curves (see Theorem B below). Under a quite weak growth condition on $H$, a characteristic curve $\Gamma$ reaches a singular point $p_{0}$ in a finite arc-length parameter and has an approximate tangent. From the opposite direction, we find another characteristic curve $\Gamma^{\prime}$ reaching also $p_{0}$ with the opposite approximate tangent. The union of $\Gamma, p_{0}$, and $\Gamma^{\prime}$ forms a smooth curve (see Corollary 3.6 and Theorem 3.10). Making use of such extension theorems, we can easily deal with the singular set, in order to study the Bernstein problem. Namely, we study entire p-minimal graphs (a graph or a solution is called entire if it is defined on the whole $x y$-plane). The following are two families of such examples (cf. [Pau]):

$$
\begin{aligned}
& u=a x+b y+c(\text { a plane with } a, b, c \text { being real constants }) \\
& u=-a b x^{2}+\left(a^{2}-b^{2}\right) x y+a b y^{2}+g(-b x+a y) \\
& \quad\left(a, b \text { being real constants such that } a^{2}+b^{2}=1 \text { and } g \in C^{2}\right) .
\end{aligned}
$$

We have the following classification result (see Section 4).

Theorem A. (1.1) and (1.2) are the only entire $C^{2}$ smooth solutions to the p-minimal graph equation (pMGE).

To prove Theorem A, we analyze the characteristic curves and the singular set of a solution for the case $H=0$. Observe that the characteristic curves are straight lines which intersect at singular points. Let $S(u)$ denote the singular set consisting of all points where $u_{x}-y=0$ and $u_{y}+x=0$. Let $N(u)$ denote the $x y$-plane projection of the negative Legendrian normal $-e_{2}$. It follows that $N(u)=\left(u_{x}-y, u_{y}+x\right) / D$ where $D=\sqrt{\left(u_{x}-y\right)^{2}+\left(u_{y}+x\right)^{2}}$. On the nonsingular domain, (pMGE) has the form

$$
\operatorname{div} N(u)(=H)=0,
$$


where div denotes the $x y$-plane divergence. The following result gives a local description of the singular set (see Section 3).

Theorem B. Let $\Omega$ be a domain in the xy-plane. Let $u \in C^{2}(\Omega)$ be such that $\operatorname{div} N(u)=H$ in $\Omega \backslash S(u)$. Suppose $|H| \leq C \frac{1}{r}$ near a singular point $p_{0} \in S(u)$ where $r(p)=\left|p-p_{0}\right|$ for $p \in \Omega$ and $C$ is a positive constant. Then either $p_{0}$ is isolated in $S(u)$ or there exists a small neighborhood of $p_{0}$ which intersects with $S(u)$ in exactly a $C^{1}$ smooth curve through $p_{0}$.

We show that the restriction on $H$ is necessary by giving a $C^{\infty}$ smooth counterexample. In additon the blow-up rate $H=C \frac{1}{r}$ is realized by some natural examples (see Section 3). Theorem B follows from a characterization for a singular point to be non-isolated (see Theorem 3.3). We remark that the local result Theorem 3.3 and hence Theorem B continue to hold when the background Heisenberg group is replaced by a general pseudohermitian manifold (see the arguments before the proof of Theorem E in Section 7).

When two characteristic lines meet at a point of a singular curve, they must form a straight line (see Lemma 4.4). So we can describe all possible configurations of characteristic lines as if singular curves are not there. It turns out that there are only two possible configurations of characteristic lines. Either all characteristic lines intersect at one singular point or they are all parallel. In the former case, we are led to the solution (1.1) while for the latter case, (1.2) is the only possible solution.

The characteristic curves on a p-minimal surface are the Legendrian geodesics (see (2.1)). Since the Legendrian geodesics in $H_{1}$ are nothing but straight lines, a general complete p-minimal surface is a complete ruled surface generated by Legendrian rulings. We will discuss this and point out that a known complete embedded non-planar p-minimal surface has no singular points (characteristic points in the terminology of some other authors) after (4.10) in Section 4.

Since (pMGE) is also a degenerate elliptic partial differential equation, one can use non-degenerate elliptic equations to approximate it. With this regularization method, Pauls ([Pau]) obtained a $W^{1, p}$ Dirichlet solution and showed that such surfaces are the X-minimal surfaces in the sense of Garofalo and Nhieu ([GN]). In general the solution to the Dirichlet problem may not be unique. However, we can still establish a uniqueness theorem by making use of a structural equality of "elliptic" type (Lemma 5.1). More generally we have the following comparison principle.

Theorem C. For a bounded domain $\Omega$ in the xy-plane, let $u, v \in C^{2}(\Omega) \cap C^{0}(\bar{\Omega})$ satisfy $\operatorname{div} N(u) \geq \operatorname{div} N(v)$ in $\Omega \backslash S$ and $u \leq v$ on $\partial \Omega$ where $S=S(u) \cup S(v)$. Suppose $\mathcal{H}_{1}(\bar{S})$, the 1-dimensional Hausdorff measure of $\bar{S}$, vanishes. Then $u \leq v$ in $\Omega$.

As an immediate consequence of Theorem $\mathrm{C}$, we have the following uniqueness result for the Dirichlet problem of (pMGE) (see Section 5). 
Corollary D. For a bounded domain $\Omega$ in the $x y$-plane, let $u, v \in C^{2}(\Omega) \cap C^{0}(\bar{\Omega})$ satisfy $\operatorname{div} N(u)=\operatorname{div} N(v)=0$ in $\Omega \backslash S$ and $u=v$ on $\partial \Omega$ where $S=S(u) \cup S(v)$. Suppose $\mathcal{H}_{1}(\bar{S})$, the 1-dimensional Hausdorff measure of $\bar{S}$, vanishes. Then $u=v$ in $\Omega$.

We remark that the condition on $\mathcal{H}_{1}(\bar{S})$ in Corollary D is necessary. A counterexample is given in [Pau] with $\mathcal{H}_{1}(\bar{S}) \neq 0$. We generalize Theorem $\mathrm{C}$ to higher dimensions and for a class of general $N$ (see Section 5). It is noticeable that we do not need the condition on the size control of the singular set for the higher dimensional version of Theorem $\mathrm{C}$ (see Theorem $\mathrm{C}^{\prime}$ ).

We also study closed p-minimal surfaces in the standard pseudohermitian 3-sphere. A characteristic curve of such a p-minimal surface is part of a Legendrian great circle (see Lemma 7.1). Using this fact, we can describe the extension theorems (Corollary 3.6, Theorem 3.10 or Lemma 7.3) in terms of Legendrian great circles, and hence give a direct proof of the nonexistence of hyperbolic p-minimal surfaces embedded in the standard pseudohermitian 3 -sphere (part of Corollary F). Then we generalize to the situation that the ambient pseudohermitian 3-manifold is arbitrary and the immersed surface has bounded p-mean curvature (see Section 7).

Theorem E. Let $M$ be a pseudohermitian 3-manifold. Let $\Sigma$ be a closed, connected surface, $C^{2}$ smoothly immersed in $M$ with bounded p-mean curvature. Then the genus of $\Sigma$ is less than or equal to 1 . In particular, there are no constant p-mean curvature or p-minimal surfaces $\Sigma$ of genus greater than one in $M$.

The idea of the proof for Theorem $\mathrm{E}$ goes as follows. A pseudohermitian manifold can be approximated locally by the Heisenberg group using the normal coordinates in the sense of Jerison and Lee ([JL2]). So we can extend the theory of singular set in Section 3 to the general situation (see Section 7). We can then apply (generalized) Lemma 3.8 to conclude that the characteristic line field has index +1 at every isolated singular point. Therefore the Euler characteristic number (equals the index sum), hence the genus, of $\Sigma$ has constraint in view of the Hopf index theorem for a line field.

Corollary F. There are no closed, connected, $C^{2}$ smoothly immersed constant pmean curvature or p-minimal surfaces of genus $\geq 2$ in the standard pseudohermitian 3-sphere.

Note that in the standard Euclidean 3-sphere, there exist many closed $C^{\infty}$ smoothly embedded minimal surfaces of genus $\geq 2$ ([La]).

On a surface in a pseudohermitian 3-manifold, we define an operator, called the tangential sublaplacian. The p-mean curvature is related to this operator acting on coordinate functions (see (2.19a), (2.19b), (2.19c)) for a graph in $H_{1}$. We therefore obtain a "normal form" (see (2.20)) of (pMGE). We also interpret the notion of p-mean curvature in terms of calibration geometry. From this we deduce the area-minimizing property for p-minimal surfaces (see Proposition 6.2). Since the second variation formula is important for later development, we derive it and discuss the stability of a p-minimal surface in Sections 6 and 7. 
We remark that, in the preprint ([GP1]), the authors claim the vertical planes are the only complete p-minimal graphs having no singular points (non-characteristic complete minimal graphs in their terminology). This is faulty. For instance, $y=x z$ is a complete (in fact, entire) p-minimal graph over the $x z$-plane and has no singular points. In $[\mathrm{CH}]$, two of us classify all the entire p-minimal graphs over any plane among other things. After our paper was completed, we were informed that the above claim had been corrected and some similar results are also obtained in the new preprint ([GP2]).

ACKNOWLEDGMENTS. We would like to thank Ai-Nung Wang for informative discussion of Monge's ([Mo]) third order equation for ruled surfaces (see Section 4). The first author would also like to thank Yng-Ing Lee for showing him some basic facts in calibration geometry. We began this research during the first author's visit at the IAS, Princeton in the 2001-2002 academic year. He would therefore like to thank the faculty and staff there as their hospitality has greatly facilitated this collaboration.

\section{Surfaces in a 3-dimensional pseudohermitian manifold}

Let $(M, J, \Theta)$ be a 3-dimensional oriented pseudohermitian manifold with a $C R$ structure $J$ and a global contact form $\Theta$ (see the Appendix). Let $\Sigma$ be a surface contained in $M$. The singular set $S_{\Sigma}$ consists of those points where the contact bundle $\xi \equiv \operatorname{ker} \Theta$ coincides with the tangent bundle $T \Sigma$ of $\Sigma$. It is easy to see that $S_{\Sigma}$ is a closed set. On the nonsingular (open) set $\Sigma \backslash S_{\Sigma}$, we call the leaves of the 1-dimensional foliation $\xi \cap T \Sigma$ the characteristic curves. These curves will play an important role in our study. On $\xi$, we can associate a natural metric $G=\frac{1}{2} d \Theta(\cdot, J \cdot)$, called the Levi metric. For a vector $v \in \xi$, we define the length of $v$ by $|v|_{G}=(G(v, v))^{1 / 2}$. With respect to this metric, we can take a unit vector field $e_{1} \in \xi \cap T \Sigma$ on $\Sigma \backslash S_{\Sigma}$, called the characteristic field. Also associated to $(J, \Theta)$ is the so-called pseudohermitian connection, denoted as $\nabla^{p . h}$. (see (A.2) in the Appendix). We can define a notion of mean curvature for $\Sigma$ in this geometry as follows. Since $\nabla^{p . h}$. preserves the Levi metric $G, \nabla^{p . h} e_{1}$ is perpendicular to $e_{1}$ with respect to $G$. On the other hand, it is obvious that $G\left(e_{1}, e_{2}\right)=0$ where $e_{2}=J e_{1}$. We call $e_{2}$ the Legendrian normal or Gauss map. So we have

$$
\nabla_{e_{1}}^{p . h .} e_{1}=H e_{2}
$$

for some function $H$. We call $H$ the p(pseudohermitian)-mean curvature of $\Sigma$. Note that if we change the sign of $e_{1}$, then $e_{2}$ and $H$ change signs accordingly. If $H=0$, we call $\Sigma$ a p-minimal surface. In this situation the characteristic curves are nothing but Legendrian (i.e., tangent to $\xi$ ) geodesics with respect to the pseudohermitian connection. 
We are going to give a variational formulation for the p-mean curvature $H$. First let us find a candidate area integral. Suppose $\Omega$ is a smooth domain in $M$ with boundary $\partial \Omega=\Sigma$. Consider $V(\Omega)$, the volume of $\Omega$, given by

$$
V(\Omega)=\frac{1}{2} \int_{\Omega} \Theta \wedge d \Theta
$$

( $\frac{1}{2}$ is a normalization constant. For $\Omega \subset H_{1}$, this volume is just the usual Euclidean volume). Take Legendrian fields $e_{1}, e_{2}=J e_{1} \in \xi$, orthonormal with respect to $G$, wherever defined in a neighborhood of $\Sigma$ (note that we do not require $e_{1}$ to be characteristic, i.e. tangent along $\Sigma$ here). We consider a variation of the surface $\Sigma$ in the direction $f e_{2}$ where $f$ is a suitable function with compact support in $\Sigma \backslash S_{\Sigma}$. The vector field $f e_{2}$ generates a flow $\varphi_{t}$ for $t$ close to 0 . We compute

$$
\begin{aligned}
\delta_{f e_{2}} V(\Omega) & =\left.\frac{d}{d t}\right|_{t=0} V\left(\varphi_{t}(\Omega)\right)=\left.\frac{1}{2} \frac{d}{d t}\right|_{t=0} \int_{\Omega} \varphi_{t}^{*}(\Theta \wedge d \Theta) \\
& =\frac{1}{2} \int_{\Omega} L_{f e_{2}}(\Theta \wedge d \Theta) .
\end{aligned}
$$

It follows from the formula $L_{X}=d \circ i_{X}+i_{X} \circ d\left(i_{X}\right.$ denotes the interior product in the direction $X$ ) that

$$
L_{f e_{2}}(\Theta \wedge d \Theta)=d \circ i_{f e_{2}}(\Theta \wedge d \Theta) .
$$

Substituting (2.3) in (2.2) and making use of Stokes' theorem, we obtain

$$
\delta_{f e_{2}} V(\Omega)=\frac{1}{2} \int_{\Sigma} i_{f e_{2}}(\Theta \wedge d \Theta)=\int_{\Sigma} f \Theta \wedge e^{1}
$$

Here $e^{1}$ together with $e^{2}, \Theta$ form a dual basis of $\left(e_{1}, e_{2}, T\right)$ where $T$ is the Reeb vector field (uniquely determined by $\Theta(T)=1$ and $i_{T} d \Theta=0$ ). Note that $d \Theta=2 e^{1} \wedge e^{2}$ (see (A.1r)). For $e_{1}$ being a characteristic field, we define the p-area of a surface $\Sigma$ to be the surface integral of the 2 -form $\Theta \wedge e^{1}$ :

$$
\text { p-Area }(\Sigma)=\int_{\Sigma} \Theta \wedge e^{1}
$$

Note that $\Theta \wedge e^{1}$ continuously extends over the singular set $S_{\Sigma}$ and vanishes on $S_{\Sigma}$. In fact, we can write $e^{1}$ with respect to a dual orthonormal basis $\left\{\hat{e}^{1}, \hat{e}^{2}\right\}$ of $\xi$, which is smooth near a singular point, say $p_{0}$, as follows: $e^{1}=\cos \beta \hat{e}^{1}$ $+\sin \beta \hat{e}^{2}$. Here $\beta$ may not be continuous at $p_{0}$. Now $\Theta \wedge \hat{e}^{1}$ and $\Theta \wedge \hat{e}^{2}$ tend to 0 on $\Sigma$ as $p \in \Sigma$ tends to $p_{0}$ since $\Theta$ vanishes on $T_{p_{0}} \Sigma=\xi_{p_{0}}$. It follows that $\Theta \wedge e^{1}$ tends to 0 on $\Sigma$ as $p \in \Sigma$ tends to $p_{0}$ since $\cos \beta$ and $\sin \beta$ are bounded by 1 . 
We can recover the p-mean curvature $H$ from the first variation formula of the p-area functional (2.5). We compute

$$
\delta_{f e_{2}} \int_{\Sigma} \Theta \wedge e^{1}=\int_{\Sigma} L_{f e_{2}}\left(\Theta \wedge e^{1}\right)=\int_{\Sigma} i_{f e_{2}} \circ d\left(\Theta \wedge e^{1}\right) .
$$

Here we have used the formula $L_{X}=d \circ i_{X}+i_{X} \circ d$ and the condition that $f$ is a function with compact support away from the singular set and the boundary of $\Sigma$. From the equations $d \Theta=2 e^{1} \wedge e^{2}$ and $d e^{1}=-e^{2} \wedge \omega \bmod \Theta$ (see (A.1r), (A.3r)), we compute

$$
d\left(\Theta \wedge e^{1}\right)=d \Theta \wedge e^{1}-\Theta \wedge d e^{1}=\Theta \wedge e^{2} \wedge \omega .
$$

Substituting (2.7) into (2.6), we obtain by the definition of the interior product that

$$
\begin{aligned}
\delta_{f e_{2}} \int_{\Sigma} \Theta \wedge e^{1} & =\int_{\Sigma} f\left(-\Theta \wedge \omega+\omega\left(e_{2}\right) \Theta \wedge e^{2}\right) \\
& =\int_{\Sigma}-f \omega\left(e_{1}\right) \Theta \wedge e^{1} \\
\left(\Theta \wedge e^{2}\right. & \left.=0 \text { on } \Sigma \text { since } e_{1} \text { is tangent along } \Sigma\right) \\
& =-\int_{\Sigma} f H \Theta \wedge e^{1} .
\end{aligned}
$$

In the last equality, we have used the fact that $H=\omega\left(e_{1}\right)$ (obtained by comparing (2.1) with (A.2r)). Similarly we can also compute the first variation of (2.5) with respect to the field $g T$ where $g$ is a function with compact support away from the singular set and the boundary of $\Sigma$. Together with (2.8), the result reads

$$
\delta_{f e_{2}+g T} \int_{\Sigma} \Theta \wedge e^{1}=-\int_{\Sigma}(f-\alpha g) H \Theta \wedge e^{1} .
$$

Here we define the function $\alpha$ on $\Sigma \backslash S_{\Sigma}$ such that $\alpha e_{2}+T \in T \Sigma$. We leave the deduction of $\left(2.8^{\prime}\right)$ to the reader. Let $\psi$ be a defining function of $\Sigma$. It follows that the subgradient $\nabla_{b} \psi=\left(e_{1} \psi\right) e_{1}+\left(e_{2} \psi\right) e_{2}=\left(e_{2} \psi\right) e_{2}$ since $e_{1} \in T \Sigma$, hence $e_{1} \psi=0$. So $\nabla_{b} \psi /\left|\nabla_{b} \psi\right|_{G}=e_{2}$ (change the sign of $\psi$ if necessary). Next we compute the subdivergence of $e_{2}$. Since $\nabla^{p . h}$. preserves the Levi metric $G$, we can easily obtain $G\left(\nabla^{p . h \cdot} e_{2}, e_{2}\right)=0$ and $G\left(\nabla_{e_{1}}^{p . h} \cdot e_{2}, e_{1}\right)=-G\left(e_{2}, \nabla_{e_{1}}^{p . h} \cdot e_{1}\right)=$ $-H$ by (2.1). Therefore $\operatorname{div}_{b}\left(e_{2}\right) \equiv G\left(\nabla_{e_{1}}^{p . h} \cdot e_{2}, e_{1}\right)+G\left(\nabla_{e_{2}}^{p . h} \cdot e_{2}, e_{2}\right)=-H$. We have derived the p-mean curvature equation (pMCE) and the p-minimal surface equation (pMSE):

$$
-\operatorname{div}_{b}\left(\nabla_{b} \psi /\left|\nabla_{b} \psi\right|_{G}\right)=H
$$

and

$$
\operatorname{div}_{b}\left(\nabla_{b} \psi /\left|\nabla_{b} \psi\right|_{G}\right)=0,
$$

respectively. 
For a graph $(x, y, u(x, y))$ in the 3-dimensional Heisenberg group $H_{1}$, we can take $\psi=z-u(x, y)$. Then (at a nonsingular point) up to sign, $e_{1}$ is uniquely determined by the following equations:

$$
\begin{aligned}
\Theta_{0} & \equiv d z+x d y-y d x=0, \\
d \psi & =d(z-u(x, y))=0, \\
G\left(e_{1}, e_{1}\right) & =\frac{1}{2} d \Theta_{0}\left(e_{1}, J e_{1}\right)=1 .
\end{aligned}
$$

Using (2.9a), (2.9c), we can write $e_{1}=f \hat{e}_{1}+g \hat{e}_{2}$ with $f^{2}+g^{2}=1$, in which $\hat{e}_{1}=\frac{\partial}{\partial x}+y \frac{\partial}{\partial z}, \hat{e}_{2}=\frac{\partial}{\partial y}-x \frac{\partial}{\partial z}$ are standard left-invariant Legendrian vector fields in $H_{1}$ (see the Appendix). Applying (2.9b) to this expression, we obtain $\left(u_{x}-\right.$ y) $f+\left(u_{y}+x\right) g=0$. So $(f, g)= \pm\left(-\left(u_{y}+x\right), u_{x}-y\right) /\left[\left(u_{x}-y\right)^{2}+\left(u_{y}+x\right)^{2}\right]^{1 / 2}$ (positive sign so that $\nabla_{b} \psi /\left|\nabla_{b} \psi\right|_{G}=e_{2}=-\left[\left(u_{x}-y\right) \hat{e}_{1}+\left(u_{y}+x\right) \hat{e}_{2}\right] / D$ where $\left.D=\left[\left(u_{x}-y\right)^{2}+\left(u_{y}+x\right)^{2}\right]^{1 / 2}\right)$. Now from (pMCE) we obtain a formula for $H$ through a direct computation:

$$
H=D^{-3}\left\{\left(u_{y}+x\right)^{2} u_{x x}-2\left(u_{y}+x\right)\left(u_{x}-y\right) u_{x y}+\left(u_{x}-y\right)^{2} u_{y y}\right\} .
$$

At a nonsingular point, the equation (pMSE) reduces to the p-minimal graph equation (pMGE):

$$
\left(u_{y}+x\right)^{2} u_{x x}-2\left(u_{y}+x\right)\left(u_{x}-y\right) u_{x y}+\left(u_{x}-y\right)^{2} u_{y y}=0 .
$$

In fact, if $u$ is $C^{2}$ smooth, the p-mean curvature $H$ in (2.10) vanishes on the nonsingular domain (where $D \neq 0$ ) if and only if (pMGE) holds on the whole domain. We can also compute $e^{1}=D^{-1}\left\{-\left(u_{y}+x\right) d x+\left(u_{x}-y\right) d y\right\}$ and express the p-area 2-form as follows:

$$
\Theta \wedge e^{1}=D d x \wedge d y=\left[\left(u_{x}-y\right)^{2}+\left(u_{y}+x\right)^{2}\right]^{1 / 2} d x \wedge d y .
$$

At a singular point, the contact form $\Theta$ is proportional to $d \psi$ (see (2.9a), (2.9b)). Therefore $u_{x}-y=0, u_{y}+x=0$ describe the $x y$-plane projection $S(u)$ of the singular set $S_{\Sigma}$ :

$$
S(u)=\left\{(x, y) \in R^{2}: u_{x}-y=0, u_{y}+x=0\right\} .
$$

From (2.11) we see that the p-area form $\Theta \wedge e^{1}$ is degenerate on $S(u)$ or $S_{\Sigma}$.

Let $e_{2}$ be the Legendrian normal of a family of deformed surfaces foliating a neighborhood of $\Sigma$. We define the tangential subgradient $\nabla_{b}^{t}$ of a function $f$ defined near $\Sigma$ by the formula: $\nabla_{b}^{t} f=\nabla_{b} f-G\left(\nabla_{b} f, e_{2}\right) e_{2}$ (see (A.8) for the definition of $\nabla_{b} f$ ) and the tangential pseudohermitian connection $\nabla^{t . p . h}$. of a Legendrian (i.e. in $\xi$ ) vector field $X$ by $\nabla^{t \cdot p \cdot h \cdot} X=\nabla^{p \cdot h} X-G\left(\nabla^{p \cdot h \cdot} X, e_{2}\right) e_{2}$. Then we define the tangential sublaplacian $\Delta_{b}^{t}$ of $f$ by

$$
\Delta_{b}^{t} f=\operatorname{div}_{b}^{t}\left(\nabla_{b}^{t} f\right)
$$


where $\operatorname{div}_{b}^{t}(X)$ is defined to be the trace of $\nabla^{t \cdot p . h} X$ considered as an endomorphism of $\xi: v \rightarrow \nabla_{v}^{t . p . h} X$. Now for an orthonormal basis $e_{1}, e_{2}$ of $\xi$ with respect to $G$, we have

$$
\operatorname{div}_{b}^{t}(X)=G\left(\nabla_{e_{1}}^{t \cdot p \cdot h \cdot} X, e_{1}\right)+G\left(\nabla_{e_{2}}^{t . p . h \cdot} X, e_{2}\right)=G\left(\nabla_{e_{1}}^{t \cdot p . h} \cdot X, e_{1}\right)
$$

since $\nabla_{e_{2}}^{t \cdot p h} X$ is proportional to $e_{1}$. On the other hand, we write $\nabla_{b} f=$ $\left(e_{1} f\right) e_{1}+\left(e_{2} f\right) e_{2}$ (cf. (A.8r)). It follows that $\nabla_{b}^{t} f=\left(e_{1} f\right) e_{1}$. Setting $X=$ $\nabla_{b}^{t} f=\left(e_{1} f\right) e_{1}$ in (2.13) and noting that $\nabla_{e_{1}}^{t . p . h} \cdot e_{1}=0$ by (2.1) gives $\operatorname{div}_{b}^{t}\left(\nabla_{b}^{t} f\right)=$ $\left(e_{1}\right)^{2} f+\left(e_{1} f\right) G\left(\nabla_{e_{1}}^{t . p . h} \cdot e_{1}, e_{1}\right)=\left(e_{1}\right)^{2} f$. Substituting this in (2.12), we obtain

$$
\Delta_{b}^{t} f=\left(e_{1}\right)^{2} f .
$$

Note that (2.14) holds for a general surface $\Sigma$ contained in an arbitrary pseudohermitian 3-manifold. We also note that $\Delta_{b}^{t}+2 \alpha e_{1}$ is self-adjoint with respect to the p-area form $\Theta \wedge e^{1}$ as shown by the following integral formula:

$$
\int_{\Sigma}\left[f \Delta_{b}^{t} g-g \Delta_{b}^{t} f\right] \Theta \wedge e^{1}=2 \int_{\Sigma}\left[g\left(e_{1} f\right)-f\left(e_{1} g\right)\right] \alpha \Theta \wedge e^{1}
$$

for smooth functions $f, g$ with compact support away from the singular set. The proof is left to the reader (Hint: observe that the adjoint of $e_{1}$ is $-e_{1}-2 \alpha$ by noting that $\Theta \wedge e^{2}=0$ and $e^{1} \wedge e^{2}=\alpha e^{1} \wedge \Theta$ on the surface). When $\Sigma$ is a graph $(x, y, u(x, y))$ in $H_{1}$, we can relate $\left(e_{1}\right)^{2} f$ for $f=x, y$, or $u$ to the p-mean curvature $H$. Denote the projection of $-e_{2}\left(-e_{1}\right.$, respectively) onto the $x y$-plane by $N(u)$ or simply $N\left(N^{\perp}(u)\right.$ or simply $N^{\perp}$, respectively). Recall that $e_{1}=\left[-\left(u_{y}+x\right) \hat{e}_{1}+\left(u_{x}-y\right) \hat{e}_{2}\right] / D$ where $D=\left[\left(u_{x}-y\right)^{2}+\left(u_{y}+x\right)^{2}\right]^{1 / 2}$ (see the paragraph after (2.9)). So $N^{\perp}=\left[\left(u_{y}+x\right) \partial_{x}-\left(u_{x}-y\right) \partial_{y}\right] / D$. Write $\left(u_{y}+x\right) D^{-1}=\sin \theta,\left(u_{x}-y\right) D^{-1}=\cos \theta$ for some local function $\theta$. Then we can write

$$
\begin{aligned}
N & =(\cos \theta) \partial_{x}+(\sin \theta) \partial_{y}, \\
N^{\perp} & =(\sin \theta) \partial_{x}-(\cos \theta) \partial_{y} .
\end{aligned}
$$

First note that from (pMCE) we can express the p-mean curvature $H$ as follows:

$$
H=\operatorname{div} N=(\cos \theta)_{x}+(\sin \theta)_{y}=-(\sin \theta) \theta_{x}+(\cos \theta) \theta_{y} .
$$

Now starting from (2.15b) and using (2.16), we can deduce

$$
\begin{aligned}
\left(N^{\perp}\right)^{2}= & \sin ^{2} \theta \partial_{x}^{2}-2 \sin \theta \cos \theta \partial_{x} \partial_{y}+\cos ^{2} \theta \partial_{y}^{2} \\
& -(\cos \theta) H \partial_{x}-(\sin \theta) H \partial_{y} .
\end{aligned}
$$

On the other hand, we can write (2.10) in the following form:

$$
H=D^{-1}\left(\sin ^{2} \theta \partial_{x}^{2} u-2 \sin \theta \cos \theta \partial_{x} \partial_{y} u+\cos ^{2} \theta \partial_{y}^{2} u\right) .
$$


Applying (2.17) to $x, y, u(x, y)$, respectively and making use of (2.18), we obtain

$$
\begin{aligned}
\Delta_{b}^{t} x & =\left(e_{1}\right)^{2} x=\left(N^{\perp}\right)^{2} x=-(\cos \theta) H, \\
\Delta_{b}^{t} y & =\left(e_{1}\right)^{2} y=\left(N^{\perp}\right)^{2} y=-(\sin \theta) H, \\
\Delta_{b}^{t} u & =\left(e_{1}\right)^{2} u=\left(N^{\perp}\right)^{2} u=D^{-1}\left(x u_{y}-y u_{x}+x^{2}+y^{2}\right) H \\
& =H(x \sin \theta-y \cos \theta)=H \operatorname{det}\left[\begin{array}{cc}
x & y \\
\cos \theta & \sin \theta
\end{array}\right]
\end{aligned}
$$

(here "det" means determinant). Formula (2.19c) gives the following normal form of (pMGE):

Lemma 2.1. For a $C^{2}$ smooth p-minimal graph $u=u(x, y)$ in the 3-dimensional Heisenberg group $H_{1}$, we have the equation

$$
\Delta_{b}^{t} u=\left(e_{1}\right)^{2} u=\left(N^{\perp}\right)^{2} u=0
$$

on the nonsingular domain.

Also from (2.19a) and (2.19b), we have $\Delta_{b}^{t} x=\Delta_{b}^{t} y=0$ on a p-minimal graph $\Sigma=\{(x, y, u(x, y))\}$. Together with $(2.20)$, we have $\Delta_{b}^{t}(x, y, z) \equiv$ $\left(\Delta_{b}^{t} x, \Delta_{b}^{t} y, \Delta_{b}^{t} z\right)=(0,0,0)$ (i.e., $\Delta_{b}^{t}$ annihilates the coordinate functions) on $\Sigma$. This is a property analogous to that for (Euclidean) minimal surfaces in $\mathbb{R}^{3}$ ([Os]). In general, we have

$$
\Delta_{b}^{t}(x, y, z)=H e_{2} .
$$

We will often call the $x y$-plane projection of characteristic curves for a graph $(x, y, u(x, y))$ in $H_{1}$ still characteristic curves if no confusion occurs. Note that the integral curves of $N^{\perp}$ are just the $x y$-plane projection of integral curves of $e_{1}$. So they are characteristic curves. Along a characteristic curve $(x(s), y(s))$ where $s$ is a unit-speed parameter, we have the equations

$$
\frac{d x}{d s}=\sin \theta, \quad \frac{d y}{d s}=-\cos \theta
$$

by (2.15b). Noting that $u_{x}=(\cos \theta) D+y, u_{y}=(\sin \theta) D-x$, we compute

$$
\begin{aligned}
\frac{d u}{d s} & =u_{x} \frac{d x}{d s}+u_{y} \frac{d y}{d s}=[(\cos \theta) D+y] \sin \theta+[(\sin \theta) D-x](-\cos \theta) \\
& =x \cos \theta+y \sin \theta \\
\frac{d \theta}{d s} & =\theta_{x} \frac{d x}{d s}+\theta_{y} \frac{d y}{d s}=\theta_{x} \sin \theta-\theta_{y} \cos \theta=-H
\end{aligned}
$$


by (2.16). In general, we can consider $H$ as a function of $x, y, u, \theta$ in view of the O.D.E. system (2.21), (2.22) and (2.23). From (2.21) and (2.23), we compute

$$
\frac{d^{2} x}{d s^{2}}=-H \cos \theta, \quad \frac{d^{2} y}{d s^{2}}=-H \sin \theta .
$$

Observe that $(\cos \theta, \sin \theta)$ is the unit normal to the unit tangent $\left(\frac{d x}{d s}, \frac{d y}{d s}\right)=(\sin \theta$, $-\cos \theta$ ). So $-H$ is just the curvature of a characteristic curve. In particular, when $H=0$, characteristic curves are nothing but straight lines or line segments (see Proposition 4.1 and Corollary 4.2).

\section{The singular set-proof of Theorem B}

Let $\Omega$ be a domain (connected open subset) in the $x y$-plane, and let $u \in C^{2}(\Omega)$. Let $\Sigma=\left\{(x, y, u(x, y) \mid(x, y) \in \Omega\} \subset H_{1}\right.$. In this section, we want to analyze $S(u)$ (still called the singular set), the $x y$-plane projection of the singular set $S_{\Sigma}$, for the graph $\Sigma$.

First for $a, b \in R, a^{2}+b^{2}=1$, we define $F_{a, b} \equiv a\left(u_{x}-y\right)+b\left(u_{y}+x\right)$ and $\Gamma_{a, b} \equiv\left\{(x, y) \in \Omega \mid F_{a, b}(x, y)=0\right\}$. Then it is easy to see that $\Gamma_{a, b}=$ $S(u) \cup\{(x, y) \in \Omega \mid N(u)(x, y)= \pm(b,-a)\}$. Let

$$
U=\left[\begin{array}{cc}
u_{x x} & u_{x y}-1 \\
u_{x y}+1 & u_{y y}
\end{array}\right]
$$

Lemma 3.1. Let $u \in C^{2}(\Omega)$ and let $p_{0} \in S(u) \subset \Omega$. Then there exists a small neighborhood of $p_{0}$, whose intersection with $S(u)$ is contained in a $C^{1}$ smooth curve.

Proof. Compute the gradient of $F_{a, b}$ :

$$
\begin{aligned}
\nabla F_{a, b} & =\left(a u_{x x}+b\left(u_{x y}+1\right), a\left(u_{x y}-1\right)+b u_{y y}\right) \\
& =(a, b)\left[\begin{array}{cc}
u_{x x} & u_{x y}-1 \\
u_{x y}+1 & u_{y y}
\end{array}\right]=(a, b) U .
\end{aligned}
$$

Note that $U$ is never a zero matrix since $u_{x y}+1$ and $u_{x y}-1$ can never be zero simultaneously. So there exists at most one unit eigenvector $\left(a_{0}, b_{0}\right)$ of eigenvalue 0 up to a sign for $U$ at $p_{0}$. For $(a, b) \neq \pm\left(a_{0}, b_{0}\right), \nabla F_{a, b} \neq 0$ at $p_{0}$. Then by the implicit function theorem, there exists a small neighborhood of $p_{0}$, in which $\Gamma_{a, b}$ at least for $(a, b) \neq \pm\left(a_{0}, b_{0}\right)$ forms a $C^{1}$ smooth curve. On the other hand, it is obvious that $S(u)$ is contained in $\Gamma_{a, b}$. We are done.

Lemma 3.2. Let $u \in C^{2}(\Omega)$ and suppose $p_{0} \in S(u) \subset \Omega$ is not isolated, i.e., there exists a sequence of distinct points $p_{j} \in S(u)$ approaching $p_{0}$. Then $\operatorname{det} U\left(p_{0}\right)$ is zero. 
Proof. Let $\Gamma_{a, b}$ be the $C^{1}$ smooth curve as in the proof of Lemma 3.1. Since $\Gamma_{a, b}$ is $C^{1}$ smooth near $p_{0}$, we can take a parameter $s$ of unit speed for $\Gamma_{a, b}$ near $p_{0}$, and find a subsequence of $p_{j}$, still denoted as $p_{j}, p_{j}=(x, y)\left(s_{j}\right), p_{0}=$ $(x, y)\left(s_{0}\right)$ such that $s_{j}$ tends to $s_{0}$ monotonically. Since $\left(u_{x}-y\right)\left(p_{j}\right)=\left(u_{x}-\right.$ $y)(x, y)\left(s_{j}\right)=0$ for all $j$, there exists $\tilde{s}_{j}$ between $s_{j}$ and $s_{j+1}$ such that $d\left(u_{x}-\right.$ $y) / d s=0$ at $\tilde{s}_{j}$. So by the chain rule we obtain

$$
u_{x x} \frac{d x}{d s}+\left(u_{x y}-1\right) \frac{d y}{d s}=0
$$

at $\tilde{s}_{j}$ and at $s_{0}$ by letting $\tilde{s}_{j}$ go to $s_{0}$. Starting from $\left(u_{y}+x\right)\left(p_{j}\right)=\left(u_{y}+\right.$ $x)(x, y)\left(s_{j}\right)=0$, we also obtain by a similar argument that

$$
\left(u_{x y}+1\right) \frac{d x}{d s}+u_{y y} \frac{d y}{d s}=0
$$

at $s_{0}$. But $\left(\frac{d x}{d s}, \frac{d y}{d s}\right) \neq 0$ since $s$ is a unit-speed parameter. It follows from (3.2a), (3.2b) that $\operatorname{det} U\left(p_{0}\right)=0$.

Note that we do not assume any condition on $H$ in Lemma 3.1 and Lemma 3.2. If we make a restriction on $H$, we can obtain the converse of Lemma 3.2 .

Theorem 3.3. Let $\Omega$ be a domain in the $x y$-plane. Let $u \in C^{2}(\Omega)$ be such that $\operatorname{div} N(u)=H$ in $\Omega \backslash S(u)$. Suppose $|H| \leq C \frac{1}{r}$ near a singular point $p_{0} \in S(u)$ where $r(p)=\left|p-p_{0}\right|$ for $p \in \Omega$ and $C$ is a positive constant. Then the following are equivalent:

(1) $p_{0}$ is not isolated in $S(u)$,

(2) $\operatorname{det} U\left(p_{0}\right)=0$,

(3) there exists a small neighborhood of $p_{0}$ which intersects with $S(u)$ in exactly a $C^{1}$ smooth curve through $p_{0}$.

Proof. (1) $\Rightarrow(2)$ by Lemma 3.2. (3) $\Rightarrow(1)$ is obvious. It suffices to show that $(2) \Rightarrow(3)$. Suppose $\operatorname{det} U\left(p_{0}\right)=0$. Note that $U\left(p_{0}\right) \neq 0$-matrix since the offdiagonal terms $u_{x y}+1$ and $u_{x y}-1$ in $(U)$ can never be zero simultaneously. Therefore $\operatorname{rank}\left(U\left(p_{0}\right)\right)=1$, and there exists a unique $\left(a_{0}, b_{0}\right)$, up to sign, such that $\left(a_{0}, b_{0}\right) U=0$ at $p_{0}$. So for any $(a, b) \neq \pm\left(a_{0}, b_{0}\right), F_{a, b} \equiv a\left(u_{x}-y\right)+b\left(u_{y}+\right.$ $x)$ satisfies $\nabla F_{a, b} \neq 0$ at $p_{0}$ and for $\left(a_{1}, b_{1}\right) \neq \pm\left(a_{0}, b_{0}\right),\left(a_{2}, b_{2}\right) \neq \pm\left(a_{0}, b_{0}\right)$ and $\left(a_{1}, b_{1}\right) \neq \pm\left(a_{2}, b_{2}\right)$

$$
\nabla F_{a_{1}, b_{1}}=c \nabla F_{a_{2}, b_{2}}
$$

at $p_{0}$ where $c$ is a nonzero constant. Therefore $\Gamma_{a_{1}, b_{1}}$ and $\Gamma_{a_{2}, b_{2}}$ are $C^{1}$ smooth curves in a neighborhood of $p_{0}$ (recall that $\Gamma_{a, b}$ is defined by $F_{a, b}=0$ ). Also $\Gamma_{a_{1}, b_{1}}$ and $\Gamma_{a_{2}, b_{2}}$ are tangent at $p_{0}$. Thus we can take unit-speed arc-length parameters $s, t$ for $\Gamma_{a_{1}, b_{1}}, \Gamma_{a_{2}, b_{2}}$ described by $\gamma_{1}(s), \gamma_{2}(t)$, respectively, so that $\gamma_{1}(0)=\gamma_{2}(0)=p_{0}$ and $\gamma_{1}^{\prime}(0)=\gamma_{2}^{\prime}(0)$. Observe that

$$
\begin{aligned}
& \left(r \circ \gamma_{1}\right)^{\prime}(0+)=\left(r \circ \gamma_{2}\right)^{\prime}(0+)=1 \\
& \left(r \circ \gamma_{1}\right)^{\prime}(0-)=\left(r \circ \gamma_{2}\right)^{\prime}(0-)=-1 .
\end{aligned}
$$


Therefore we can find a small $\epsilon>0$ such that $\left(r \circ \gamma_{1}\right)^{\prime}(s),\left(r \circ \gamma_{2}\right)^{\prime}(t)>0$ for $s, t>0\left(\left(r \circ \gamma_{1}\right)^{\prime}(s),\left(r \circ \gamma_{2}\right)^{\prime}(t)<0\right.$ for $s, t<0$, respectively) whenever $\gamma_{1}(s), \gamma_{2}(t) \in B_{\epsilon}\left(p_{0}\right)$, a ball of radius $\epsilon$ and center $p_{0}$. Also note that $S(u) \cap$ $B_{\epsilon}\left(p_{0}\right) \subset \Gamma_{a_{i}, b_{i}}, i=1,2$. We can write

$$
\begin{aligned}
& \left(\Gamma_{a_{1}, b_{1}} \cap B_{\epsilon}\left(p_{0}\right)\right) \backslash S(u)=\cup_{j=1}^{\infty} \gamma_{1}(] s_{j}^{+}, \tilde{s}_{j}^{+}[) \cup \cup_{j=1}^{\infty} \gamma_{1}(] s_{j}^{-}, \tilde{s}_{j}^{-}[) \\
& \left(\Gamma_{a_{2}, b_{2}} \cap B_{\epsilon}\left(p_{0}\right)\right) \backslash S(u)=\cup_{j=1}^{\infty} \gamma_{2}(] t_{j}^{+}, \tilde{t}_{j}^{+}[) \cup \cup_{j=1}^{\infty} \gamma_{2}(] t_{j}^{-}, \tilde{t}_{j}^{-}[)
\end{aligned}
$$

where $] s_{j}^{+}, \tilde{s}_{j}^{+}[,] s_{j}^{-}, \tilde{s}_{j}^{-}[$, etc. denote open intervals and we have arranged

$$
\begin{aligned}
s_{1}^{-} & <\tilde{s}_{1}^{-} \leq \ldots s_{j}^{-}<\tilde{s}_{j}^{-} \leq s_{j+1}^{-}<\tilde{s}_{j+1}^{-} \leq \ldots \\
& \leq 0 \leq \ldots \leq s_{j+1}^{+}<\tilde{s}_{j+1}^{+} \leq s_{j}^{+}<\tilde{s}_{j}^{+} \leq \ldots \leq s_{1}^{+}<\tilde{s}_{1}^{+} \\
t_{1}^{-} & <\tilde{t}_{1}^{-} \leq \ldots t_{j}^{-}<\tilde{t}_{j}^{-} \leq t_{j+1}^{-}<\tilde{t}_{j+1}^{-} \leq \ldots \\
& \leq 0 \leq \ldots \leq t_{j+1}^{+}<\tilde{t}_{j+1}^{+} \leq t_{j}^{+}<\tilde{t}_{j}^{+} \leq \ldots \leq t_{1}^{+}<\tilde{t}_{1}^{+}
\end{aligned}
$$

(if there are only finite number of open intervals for negative (positive, respectively) $s$, then $\tilde{s}_{j}^{-}=s_{j}^{-}=\tilde{s}_{m}^{-}\left(s_{j}^{+}=\tilde{s}_{j}^{+}=s_{m}^{+}\right.$, respectively) for $j \geq m$, a certain integer, by convention. Note that $\tilde{s}_{j}^{-}$or $s_{j}^{+}$may equal 0 for some finite $j$. In this case, all the $s$ with subindex larger than $j$ equal 0 . We apply the same convention to the parameter $t$ ). Since $r \circ \gamma_{1}$ and $r \circ \gamma_{2}$ are monotonic (increasing for positive parameters and decreasing for negative parameters), we actually have $\gamma_{1}\left(s_{j}^{ \pm}\right)=\gamma_{2}\left(t_{j}^{ \pm}\right), \gamma_{1}\left(\tilde{s}_{j}^{ \pm}\right)=\gamma_{2}\left(\tilde{t}_{j}^{ \pm}\right)$except possibly $\gamma_{1}\left(s_{1}^{-}\right) \neq \gamma_{2}\left(t_{1}^{-}\right)$or $\gamma_{1}\left(\tilde{s}_{1}^{+}\right) \neq \gamma_{2}\left(\tilde{t}_{1}^{+}\right)$. Let $e_{j}=\gamma_{1}\left(s_{j}^{+}\right)$and $\tilde{e}_{j}=\gamma_{1}\left(\tilde{s}_{j}^{+}\right)$. Then either $\Gamma_{a_{1}, b_{1}}$ and $\Gamma_{a_{2}, b_{2}}$ meet at $\tilde{e}_{1}$ or $\tilde{e}_{2}$, or they do not meet in $B_{\epsilon}\left(p_{0}\right) \backslash\left\{p_{0}\right\}$ for positive parameters. In the former situation, we need to show that $e_{i}, i \geq 1$, does not converge to $p_{0}$ as $i \rightarrow \infty$ (then there is a smaller ball $B_{\epsilon^{\prime}}\left(p_{0}\right)$ such that $S(u) \cap B_{\epsilon^{\prime}}\left(p_{0}\right)$ contains $\gamma_{1}\left(\left[0, \bar{s}^{+}[)\right.\right.$for a small $\left.\bar{s}^{+}>0\right)$. Suppose it is not so. Let $\Omega_{i}$ be the region surrounded by $\Gamma_{a_{1}, b_{1}}$ and $\Gamma_{a_{2}, b_{2}}$ from $e_{i}$ to $\tilde{e}_{i}$ for all $i \geq 1$ or 2 (if $\gamma_{1}\left(\tilde{s}_{1}^{+}\right) \neq \gamma_{2}\left(\tilde{t}_{1}^{+}\right)$) (note that the curves $\Gamma_{a_{1}, b_{1}}$ and $\Gamma_{a_{2}, b_{2}}$ only meet at singular points since $\left(a_{1}, b_{1}\right) \neq \pm\left(a_{2}, b_{2}\right)$, i.e., points of the arcs $\gamma_{1}\left(\left[\tilde{s}_{i+1}^{+}, s_{i}^{+}\right]\right)=\gamma_{2}\left(\left[\tilde{t}_{i+1}^{+}, t_{i}^{+}\right]\right)$.) Observe that $\Gamma_{a_{1}, b_{1}}$ and $\Gamma_{a_{2}, b_{2}}$ asymptotically approximate the same straight line by (3.3). So the distance function $r(p) \equiv\left|p-p_{0}\right|$ is one-to-one for $p \in \Gamma_{a_{1}, b_{1}}\left(\Gamma_{a_{2}, b_{2}}\right.$, respectively) near $p_{0}$ with parameter $s>0\left(t>0\right.$, respectively) since $\left(r \circ \gamma_{1}\right)^{\prime}(s),\left(r \circ \gamma_{2}\right)^{\prime}(t)>0$ for $s, t>0$ as shown previously. Now we want to compare both sides of

$$
\oint_{\partial \Omega_{i}} N(u) \cdot v d s=\iint_{\Omega_{i}} \operatorname{div} N(u) d x d y=\iint_{\Omega_{i}} H d x d y
$$

where $v$ is the unit outward normal to $\Gamma_{a_{1}, b_{1}}$ and $\Gamma_{a_{2}, b_{2}}$. On $\Gamma_{a_{1}, b_{1}}\left(\Gamma_{a_{2}, b_{2}}\right.$, respectively), $N(u) \perp\left(a_{1}, b_{1}\right)\left(\left(a_{2}, b_{2}\right)\right.$, respectively). So $N(u)$ is a constant unit vector field along $\Gamma_{a_{1}, b_{1}}\left(\Gamma_{a_{2}, b_{2}}\right.$, respectively). On the other hand, $v$ approaches a fixed unit vector $\nu_{0}=\nabla F_{a_{1}, b_{1}}\left(p_{0}\right) /\left|\nabla F_{a_{1}, b_{1}}\left(p_{0}\right)\right|=\nabla F_{a_{2}, b_{2}}\left(p_{0}\right) /\left|\nabla F_{a_{2}, b_{2}}\left(p_{0}\right)\right|$ 
as $e_{i}, \tilde{e}_{i}$ tend to $p_{0}$ for $i$ large. It follows that $N(u) \cdot v$ tends to a constant $c_{1}= \pm\left(-b_{1}, a_{1}\right) \cdot v_{0}\left(c_{2}= \pm\left(-b_{2}, a_{2}\right) \cdot v_{0}\right.$, respectively $)$ along $\Gamma_{a_{1}, b_{1}}\left(\Gamma_{a_{2}, b_{2}}\right.$, respectively) as $i$ goes to infinity. We choose (in advance) $\left(a_{1}, b_{1}\right) \neq \pm\left(a_{2}, b_{2}\right)$ (also both $\left.\neq \pm\left(a_{0}, b_{0}\right)\right)$ such that $c_{1} \neq c_{2}$. Thus we can estimate

$$
\left|\oint_{\partial \Omega_{i}} N(u) \cdot v d s\right| \geq \frac{\left|c_{1}-c_{2}\right|}{2}\left|r\left(\tilde{e}_{i}\right)-r\left(e_{i}\right)\right|
$$

for large $i$. On the other hand, we can make $\Omega_{i}$ contained in a fan-shaped region of angle $\theta_{i}$ with vertex $p_{0}$ so that $\theta_{i} \rightarrow 0$ as $i \rightarrow \infty$. We estimate

$$
\left|\iint_{\Omega_{i}} H d x d y\right| \leq\left|\int_{r\left(e_{i}\right)}^{r\left(\tilde{e}_{i}\right)}\left(\int_{0}^{\theta_{i}} \frac{C}{r} d \theta\right) r d r\right| \leq C \theta_{i}\left|r\left(\tilde{e}_{i}\right)-r\left(e_{i}\right)\right| .
$$

Substituting (3.5) and (3.6) into (3.4), we obtain

$$
\frac{\left|c_{1}-c_{2}\right|}{2}\left|r\left(\tilde{e}_{i}\right)-r\left(e_{i}\right)\right| \leq C \theta_{i}\left|r\left(\tilde{e}_{i}\right)-r\left(e_{i}\right)\right| .
$$

Hence $\frac{\left|c_{1}-c_{2}\right|}{2} \leq C \theta_{i}$. But $\theta_{i}$ tending to 0 gives $c_{1}=c_{2}$, a contradiction. Another situation is that $\Gamma_{a_{1}, b_{1}}$ and $\Gamma_{a_{2}, b_{2}}$ do not meet in a small neighborhood of $p_{0}$ except at $p_{0}$ for $s, t>0$. In this case, let $\Omega_{i}$ be the region surrounded by $\Gamma_{a_{1}, b_{1}}, \Gamma_{a_{2}, b_{2}}$, and $\partial B_{r_{i}}\left(p_{0}\right)$ for large $i$ and contained in a fan-shaped region of angle $\theta_{i}$ with vertex $p_{0}$ so that $r_{i} \rightarrow 0, \theta_{i} \rightarrow 0$ as $i \rightarrow \infty$. Observing that the arc length of $\bar{\Omega}_{i} \cap \partial B_{r_{i}}\left(p_{0}\right)$ is bounded by $\theta_{i} r_{i}$, we can reach a contradiction by a similar argument as above. For $s, t<0$, we apply a similar argument to conclude that there is a small $\epsilon^{\prime \prime}>0$ such that $S(u) \cap B_{\epsilon^{\prime \prime}}\left(p_{0}\right)$ contains $\left.\left.\gamma_{1}(] \bar{s}^{-}, 0\right]\right)$ for a small $\bar{s}^{-}<0$. Now an even smaller ball of radius $<\min \left(\epsilon^{\prime}, \epsilon^{\prime \prime}\right)$ and center $p_{0}$ will serve our purpose.

Proof of Theorem B. It is an immediate consequence of Theorem 3.3.

We remark that Theorem $\mathrm{B}$ does not hold if we remove the condition on $H$.

Example 1. Let $u=x g(y)$. Then $u_{x}=g(y), u_{y}=x g^{\prime}(y)$. It follows that the singular set $S(u)=\left\{g(y)=y\right.$ and $\left.g^{\prime}(y)+1=0\right\} \cup\{g(y)=y$ and $x=0\}$. Take $g(y)=\exp \left(-\frac{1}{y^{2}}\right) \sin \left(-\frac{1}{y}\right)+y$. Compute $g^{\prime}(y)=2 \exp \left(-\frac{1}{y^{2}}\right) y^{-3} \sin \left(-\frac{1}{y}\right)+$ $\exp \left(-\frac{1}{y^{2}}\right) y^{-2} \cos \left(-\frac{1}{y}\right)+1$. So for $y$ small, $g^{\prime}(y)+1=0$ has no solution. Therefore $S(u)$ (when $y$ is small) $=\{g(y)=y, x=0\}=\left\{\sin \left(-\frac{1}{y}\right)=0\right.$, $x=0\}$ has infinitely-many points near $(0,0)$. Note that $g$, hence $u$, is $C^{\infty}$ smooth. This example shows that even for $u \in C^{\infty}, S(u)$ may contain nonisolated points which do not belong to curves in $S(u)$. On the other hand, the p-mean curvature $H(u)$ has a blow-up rate $\exp \left(\frac{1}{r^{2}}\right)$ for a certain sequence of points $\left(x_{j}, y_{j}\right)$ satisfying $x_{j}=\exp \left(-\frac{1}{y_{j}^{2}}\right)$ and converging to $(0,0)$. 
Example 2. Let $u= \pm \frac{1}{2}\left(x^{2}+y^{2}\right)$. Then $u_{x}= \pm x, u_{y}= \pm y$. So $S(u)=$ $\{(0,0)\}$. By $(2.10)$ we compute the p-mean curvature $H= \pm 2^{-1 / 2} r^{-1}$ where $r=\sqrt{x^{2}+y^{2}}$. This is the case that the equality of the condition on $H$ holds.

Example 3. The following example shows that in Theorem 3.3, (2) does not imply (1) if we remove the condition on $H$. Let

$$
u=\frac{1}{2}\left(x^{2}-y^{2}\right)+\frac{1}{\beta}\left(\operatorname{sgn}(x)|x|^{\beta}-\operatorname{sgn}(y)|y|^{\beta}\right)
$$

for $\beta>2$. Compute $u_{x}=x+|x|^{\beta-1}, u_{y}=-y-|y|^{\beta-1}$. It follows that $\left(u_{x}-y\right)-\left(u_{y}+x\right)=|x|^{\beta-1}+|y|^{\beta-1}$. So $S(u) \subset\left\{|x|^{\beta-1}+|y|^{\beta-1}=0\right\}$, and hence $S(u)=\{(0,0)\}$. This means that $(0,0)$ is an isolated singular point. Compute $u_{x x}=1+(\beta-1) \operatorname{sgn}(x)|x|^{\beta-2}, u_{x y}=0$ and $u_{y y}=-1-(\beta-1) \operatorname{sgn}(y)|y|^{\beta-2}$. It is easy to see that $\left|u_{x x}\right| \leq \beta$ and $\left|u_{y y}\right| \leq \beta$ for $|x| \leq 1,|y| \leq 1$. So by (2.10) we can estimate

$$
H \leq \frac{\left|u_{x x}\right|+\left|u_{y y}\right|}{D} \leq \frac{\beta+\beta}{\left(|x|^{\beta-1}+|y|^{\beta-1}\right) / \sqrt{2}} \leq C \frac{1}{r^{\beta-1}}
$$

near $(0,0)$ where $r=\sqrt{x^{2}+y^{2}}$. In the second inequality above, we have used the following estimate

$$
\begin{aligned}
D^{2} & \equiv\left(u_{x}-y\right)^{2}+\left(u_{y}+x\right)^{2}=\left(x+|x|^{\beta-1}-y\right)^{2}+\left(-y-|y|^{\beta-1}+x\right)^{2} \\
& \geq \frac{1}{2}\left(|x|^{\beta-1}+|y|^{\beta-1}\right)^{2}
\end{aligned}
$$

(by $\left.2\left(a^{2}+b^{2}\right) \geq(a-b)^{2}\right)$. On the other hand, we observe that $U=\left(\begin{array}{ll}1 & -1 \\ 1 & -1\end{array}\right)$ at $(0,0)$. It follows that $\operatorname{det} U=0$ at $(0,0)$.

According to Theorem B, $S(u)$ may contain some $C^{1}$ smooth curves, called singular curves. We will study the behavior of $N(u)$ near a point of a singular curve. First we show that for a graph $t=u(x, y)$ the p-minimal graph equation (pMGE) is rotationally invariant.

Lemma 3.4. Suppose $u \in C^{2}$. Let $x=a \tilde{x}-b \tilde{y}, y=b \tilde{x}+a \tilde{y}$ where $a^{2}+b^{2}=1$, and let $\tilde{u}(\tilde{x}, \tilde{y})=u(x(\tilde{x}, \tilde{y}), y(\tilde{x}, \tilde{y}))$. Then we have

$$
N(\tilde{u})=N(u)\left[\begin{array}{cc}
a & -b \\
b & a
\end{array}\right]
$$

and hence $\widetilde{\operatorname{div}} N(\tilde{u})=\operatorname{div} N(u)$ where $\widetilde{\operatorname{div}}$ denotes the plane divergence with respect to $(\tilde{x}, \tilde{y})$. 
Proof. First we observe that both $\nabla u$ (viewed as a row vector) and $(-y, x)$ satisfy the following transformation law (for a plane vector):

$$
\tilde{\nabla} \tilde{u}=(\nabla u)\left[\begin{array}{cc}
a & -b \\
b & a
\end{array}\right], \quad(-\tilde{y}, \tilde{x})=(-y, x)\left[\begin{array}{cc}
a & -b \\
b & a
\end{array}\right] .
$$

It follows that $N(u)=[\nabla u+(-y, x)] / D$ also obeys the same transformation law by noting that $D$ is invariant. Then a direct computation shows that $\widetilde{\operatorname{div}} N(\tilde{u})=$ $\operatorname{div} N(u)$.

Let $\Gamma_{s}$ be a singular curve contained in $S(u)$ for a $C^{2}$ smooth $u$ (defined on a certain domain). Let $p_{0} \in \Gamma_{s}$. Suppose there exists a ball $B_{\epsilon}\left(p_{0}\right)$ such that $\Gamma_{s} \cap B_{\epsilon}\left(p_{0}\right)$ divides $B_{\epsilon}\left(p_{0}\right)$ into two disjoint nonsingular parts (this is true if $|H| \leq C \frac{1}{r}$ near $p_{0}$ in view of Theorem B). That is to say, $B_{\epsilon}\left(p_{0}\right) \backslash\left(\Gamma_{s} \cap\right.$ $\left.B_{\epsilon}\left(p_{0}\right)\right)=B^{+} \cup B^{-}$where $B^{+}$and $B^{-}$are disjoint domains (proper open and connected).

Proposition 3.5. Suppose we have the situation as described above. Then both $N(u)\left(p_{0}^{+}\right) \equiv \lim _{p \in B^{+} \rightarrow p_{0}} N(u)(p)$ and $N(u)\left(p_{0}^{-}\right) \equiv \lim _{p \in B^{-} \rightarrow p_{0}} N(u)(p)$ exist. Moreover, $N(u)\left(p_{0}^{+}\right)=-N(u)\left(p_{0}^{-}\right)$.

Proof. By Lemma 3.4 we may assume the $x$-axis through $p_{0}=\left(x_{0}, y_{0}\right)$ is transverse to $\Gamma_{s}$. Moreover, we may assume either $u_{x x}\left(x_{0}, y_{0}\right) \neq 0$ or $\left(u_{x y}+\right.$ 1) $\left(x_{0}, y_{0}\right) \neq 0$. (Note that in (3.2a) $\frac{d y}{d s} \neq 0$ at $p_{0}$. So if $u_{x x}\left(x_{0}, y_{0}\right)=0$, then $\left(u_{x y}-1\right)\left(x_{0}, y_{0}\right)=0$. It follows that $\left(u_{x y}+1\right)\left(x_{0}, y_{0}\right)=2 \neq 0$. $)$ Let $\Gamma_{s} \cap\{y=$ $c\}=\left\{\left(x_{c}, c\right)\right\}$ for $c$ close to $y_{0}$. Since $\left(x_{c}, c\right) \in \Gamma_{s}$, we have $\left(u_{y}+x\right)\left(x_{c}, c\right)=0$ and $\left(u_{x}-y\right)\left(x_{c}, c\right)=0$. So if $u_{x x}\left(x_{0}, y_{0}\right) \neq 0$, we compute

$$
\begin{aligned}
\frac{\left(u_{y}+x\right)(x, c)}{\left(u_{x}-y\right)(x, c)} & =\frac{\left(u_{y}+x\right)(x, c)-\left(u_{y}+x\right)\left(x_{c}, c\right)}{\left(u_{x}-y\right)(x, c)-\left(u_{x}-y\right)\left(x_{c}, c\right)} \\
& =\frac{\left(x-x_{c}\right)\left(u_{x y}+1\right)\left(x_{c}^{1}, c\right)}{\left(x-x_{c}\right) u_{x x}\left(x_{c}^{2}, c\right)}
\end{aligned}
$$

(for $x_{c}^{1}, x_{c}^{2}$ between $x_{c}$ and $x$ by the mean value theorem)

$$
=\frac{\left(u_{x y}+1\right)\left(x_{c}^{1}, c\right)}{u_{x x}\left(x_{c}^{2}, c\right)} \text {. }
$$

Letting $(x, c)$ go to $\left(x_{0}, y_{0}\right)$ in (3.7), we obtain

$$
\lim _{p \in B^{+} \rightarrow p_{0}} \frac{u_{y}+x}{u_{x}-y}=\frac{u_{x y}+1}{u_{x x}}\left(p_{0}\right)=\lim _{p \in B^{-} \rightarrow p_{0}} \frac{u_{y}+x}{u_{x}-y} .
$$

Therefore by (3.8) two limits of the unit vector $N(u)=\left(u_{x}-y, u_{y}+x\right) D^{-1}$ from both sides exist, and their values can only be different by a sign. That is to say,

$$
N(u)\left(p_{0}^{+}\right)= \pm N(u)\left(p_{0}^{-}\right) .
$$


Now we observe that $\left(u_{x}-y\right)\left(x, y_{0}\right)-\left(u_{x}-y\right)\left(x_{0}, y_{0}\right)=u_{x x}\left(\eta, y_{0}\right)\left(x-x_{0}\right)$ for some $\eta$ between $x_{0}$ and $x$. Since $\left(u_{x}-y\right)\left(x_{0}, y_{0}\right)=0,\left(u_{x}-y\right)\left(x, y_{0}\right)$ and $u_{x x}\left(x_{0}, y_{0}\right)$ have the same (different, respectively) sign for $x>x_{0}\left(x<x_{0}\right.$, respectively) and $x$ being close enough to $x_{0}$. Thus we should have the negative sign in (3.9). We are done. In case $u_{x x}\left(x_{0}, y_{0}\right)=0$, we have $\left(u_{x y}+1\right)\left(x_{0}, y_{0}\right) \neq$ 0 . So we compute $\frac{\left(u_{x}-y\right)(x, c)}{\left(u_{y}+x\right)(x, c)}$ instead of $\frac{\left(u_{y}+x\right)(x, c)}{\left(u_{x}-y\right)(x, c)}$, and get $\frac{u_{x x}}{u_{x y}+1}\left(p_{0}\right)$ instead of $\frac{u_{x y}+1}{u_{x x}}\left(p_{0}\right)$ in (3.8). Then we still conclude (3.9). Instead of $\left(u_{x}-y\right)\left(x, y_{0}\right)$, we consider $\left(u_{y}+x\right)\left(x, y_{0}\right)$. A similar argument as above shows that $\left(u_{y}+x\right)\left(x, y_{0}\right)$ will have the same (different, respectively) sign as $\left(u_{x y}+1\right)\left(x_{0}, y_{0}\right)$ for $x>x_{0}$ $\left(x<x_{0}\right.$, respectively) and $x$ being close enough to $x_{0}$. So we still take the negative sign in (3.9).

Note that we do not assume any condition on $H$ in Proposition 3.5. We will study how two characteristic curves meet at a point of a singular curve. We say a characteristic curve $\Gamma \subset B^{+}$or $B^{-}$touches $\Gamma_{s}$ at $p_{0}$ if $p_{0} \in \bar{\Gamma}$, the closure of $\Gamma$ in the $x y$-plane, and touches transversally if, furthermore, $p_{0}$ is the only intersection point of the tangent line of $\Gamma_{s}$ at $p_{0}$ and the tangent line of $\Gamma$ at $p_{0}$ (which makes sense in view of Proposition 3.5).

Corollary 3.6. Suppose we have the same assumptions as in Proposition 3.5. Then there is a unique characteristic curve $\Gamma_{+} \subset B^{+}$touching $\Gamma_{s}$ at $p_{0}$ transversally (with $N^{\perp}(u)\left(p_{0}^{+}\right)$being the tangent vector of $\bar{\Gamma}_{+}$at $p_{0}$ ). Similarly there exists a unique characteristic curve $\Gamma_{-} \subset B^{-}$touching $\Gamma_{s}$ also at $p_{0}$ so that $\Gamma_{-} \cup\left\{p_{0}\right\} \cup \Gamma_{+}$ forms a $C^{1}$ smooth curve in $B_{\epsilon}\left(p_{0}\right)$.

First note that we can change the sign of $N^{\perp}(u)$ if necessary to make a $C^{0}$ characteristic (i.e. tangent to integral curves of $N^{\perp}(u)$ where $N^{\perp}(u)$ is defined) vector field $\check{N}^{\perp}(u)$ in $B_{\epsilon}\left(p_{0}\right)$ in view of Proposition 3.5. So $\bar{\Gamma}_{+}$of any characteristic curve $\Gamma_{+} \subset B^{+}$touching $\Gamma_{s}$ at $p_{0}$ must have the tangent vector $N^{\perp}(u)\left(p_{0}^{+}\right)$at $p_{0}$. To show the uniqueness of $\Gamma_{+}$and the transversality of $N^{\perp}(u)\left(p_{0}^{+}\right)$to $\Gamma_{s}$ in Corollary 3.6, we observe that

Lemma 3.7. Suppose we have the same assumptions as in Proposition 3.5. Then there hold

(1) $N^{\perp}(u)\left(p_{0}^{+}\right) U\left(p_{0}\right)=0$,

(2) $(c, d) U^{T}\left(p_{0}\right)=0$

for a nonzero vector $(c, d)$ tangent to $\Gamma_{s}$ at $p_{0}$, where we view $N^{\perp}(u)\left(p_{0}^{+}\right)$as a row vector and $U^{T}$ denotes the transpose of $U$.

Proof. Let $(x(s), y(s))$ describe $\Gamma_{s}$ so that $p_{0}=(x(0), y(0))$ and $\left(x^{\prime}(0)\right.$, $\left.y^{\prime}(0)\right)=(c, d)$. Since $u_{x}-y=0, u_{y}+x=0$ on $\Gamma_{s}$, we differentiate these two equations to get (3.2a) and (3.2b) along $\Gamma_{s}$ by the chain rule. At $s=0$, we obtain (2). From the proof of Proposition 3.5, we learn that $N(u)\left(p_{0}^{+}\right)$is proportional to $\left(u_{x x}, u_{x y}+1\right)\left(p_{0}\right)$ (if this is not a zero vector). A similar argument (L'Hôpital's rule in the $y$-direction) shows that $N(u)\left(p_{0}^{+}\right)$is also proportional 
to $\left(u_{x y}-1, u_{y y}\right)\left(p_{0}\right)$ (if this is not a zero vector). Therefore $N^{\perp}(u)\left(p_{0}^{+}\right)$is perpendicular to $\left(u_{x x}, u_{x y}+1\right)\left(p_{0}\right)$ and $\left(u_{x y}-1, u_{y y}\right)\left(p_{0}\right)$, hence (1) follows.

We will give a proof of Corollary 3.6 after the proof of Theorem 3.10.

Remark. If $u$ is not of class $C^{2}$, the extension theorem (Corollary 3.6) may fail as the following example shows. Consider the function

$$
u(x, y)= \begin{cases}-x y, & y \geq 0 \\ -x y+y^{2} \cot \vartheta, & y<0\end{cases}
$$

where $0<\vartheta<\frac{\pi}{2}$. There holds

$$
N^{\perp}(u)= \begin{cases}(0,1), & y>0 \\ (\cos \vartheta, \sin \vartheta), & y<0 .\end{cases}
$$

Note that the function $u$ is of class $C^{1,1}$ on $R^{2}$ and satisfies $\operatorname{div} N(u)=0$ in $R^{2} \backslash\{y=0\}$ where $u$ is of class $C^{2}$.

Next we want to analyze the configuration of characteristic curves near an isolated singularity. First observe that for a $C^{2}$ smooth $u$ defined on a domain $\Omega$, characteristic curves are also the integral curves of the $C^{1}$ smooth vector field $N^{\perp} D=\left(u_{y}+x,-u_{x}+y\right)$ which vanishes at singular points. We think of $N^{\perp} D$ as a mapping: $\Omega \subset R^{2} \rightarrow R^{2}$, so that the differential $d\left(N^{\perp} D\right)_{p_{0}}: R^{2} \rightarrow R^{2}$ is defined for $p_{0} \in \Omega$.

Lemma 3.8. Let $u \in C^{2}(\Omega)$. Suppose $|H|=o\left(\frac{1}{r}\right)$ (little "o") near an isolated singular point $p_{0} \in \Omega$ where $r(p)=\left|p-p_{0}\right|$. Then $d\left(N^{\perp} D\right)_{p_{0}}$ is the identity linear transformation and the index of $N^{\perp} D$ at the isolated zero $p_{0}$ is +1 . Moreover, $u_{x x}=u_{x y}=u_{y y}=0$ at $p_{0}$.

Proof. In view of (2.10), we write $H=D^{-3} P(u)$ where

$$
P(u) \equiv\left(u_{y}+x\right)^{2} u_{x x}-2\left(u_{y}+x\right)\left(u_{x}-y\right) u_{x y}+\left(u_{x}-y\right)^{2} u_{y y} .
$$

Let $p-p_{0}=(\triangle x, \Delta y), r=r(p)=\left|p-p_{0}\right|=\left[(\Delta x)^{2}+(\triangle y)^{2}\right]^{1 / 2}$. Noting that $\left(u_{y}+x\right)\left(p_{0}\right)=\left(u_{x}-y\right)\left(p_{0}\right)=0$ by the definition of a singular point, we can express

$$
\begin{aligned}
& \left(u_{y}+x\right)(p)=\left(u_{y x}+1\right)\left(p_{0}\right) \Delta x+u_{y y}\left(p_{0}\right) \Delta y+o(r), \\
& \left(u_{x}-y\right)(p)=u_{x x}\left(p_{0}\right) \Delta x+\left(u_{x y}-1\right)\left(p_{0}\right) \Delta y+o(r)
\end{aligned}
$$

for $p$ near $p_{0}$. Let $a=\left(u_{y x}+1\right)\left(p_{0}\right), b=u_{y y}\left(p_{0}\right), c=u_{x x}\left(p_{0}\right)$, and $d=$ $\left(u_{x y}-1\right)\left(p_{0}\right)$. Substituting (3.11) and (3.12) in (3.10), we compute the highest order term:

$$
\begin{aligned}
P(u)= & (a \Delta x+b \Delta y)^{2} c \\
& -(a \Delta x+b \triangle y)(c \Delta x+d \triangle y)(a+d)+(c \Delta x+d \triangle y)^{2} b+o\left(r^{2}\right) \\
= & (b c-a d)\left\{(\triangle x \Delta y)\left[\begin{array}{ll}
c & a \\
d & b
\end{array}\right]\left(\begin{array}{c}
\triangle x \\
\Delta y
\end{array}\right)\right\}+o\left(r^{2}\right) .
\end{aligned}
$$


On the other hand, substituting (3.11), (3.12) into $D^{3} \equiv\left[\left(u_{x}-y\right)^{2}+\left(u_{y}+x\right)^{2}\right]^{3 / 2}$, we obtain

$$
D^{3}=\left|\left[\begin{array}{ll}
a & b \\
c & d
\end{array}\right]\left(\begin{array}{c}
\triangle x \\
\triangle y
\end{array}\right)\right|^{3}+o\left(r^{3}\right) .
$$

Note that $b c-a d=\operatorname{det} U\left(p_{0}\right) \neq 0$ by Theorem 3.3. Letting $\triangle y=0$ and assuming $c \neq 0$, we estimate the highest order term of $H=D^{-3} P(u)$ :

$$
\frac{(b c-a d) c(\triangle x)^{2}}{\left[\left(a^{2}+c^{2}\right)(\triangle x)^{2}\right]^{3 / 2}}=\frac{(b c-a d) c(\triangle x)^{2}}{\left[a^{2}+c^{2}\right]^{3 / 2}(\triangle x)^{3}}=\frac{(b c-a d) c}{\left[a^{2}+c^{2}\right]^{3 / 2} \triangle x} .
$$

The assumption $|H|=o\left(\frac{1}{r}\right)$ forces $c=0$. On the other hand, letting $\Delta x=0$ will force $b=0$ by a similar argument. Now we can write

$$
H=\frac{-a d(a+d) \Delta x \Delta y}{\left[a^{2}(\triangle x)^{2}+d^{2}(\triangle y)^{2}\right]^{3 / 2}}+o\left(\frac{1}{r}\right) .
$$

It follows from the assumption $|H|=o\left(\frac{1}{r}\right)$ again that $a+d=0$ (note that $\left.a d=a d-b c=-\operatorname{det} U\left(p_{0}\right) \neq 0\right)$. We have proved that $u_{x x}\left(p_{0}\right)=c=0$, $u_{y y}\left(p_{0}\right)=b=0, u_{x y}\left(p_{0}\right)=\frac{a+d}{2}=0$. Therefore in matrix form,

$$
d\left(N^{\perp} D\right)_{p_{0}}=\left[\begin{array}{cc}
u_{y x}+1 & u_{y y} \\
-u_{x x} & -u_{x y}+1
\end{array}\right]=\left[\begin{array}{ll}
1 & 0 \\
0 & 1
\end{array}\right]
$$

Note that $\operatorname{det}\left(d\left(N^{\perp} D\right)_{p_{0}}\right)=1>0$. So the index of $N^{\perp} D$ at $p_{0}$ is +1 (see, e.g., Lemma 5 in Section 6 in [Mil]).

Lemma 3.9. Let $u \in C^{2}(\Omega)$. Suppose $|H|=o\left(\frac{1}{r}\right)$ (little "o") near an isolated singular point $p_{0} \in \Omega$ where $r(p)=\left|p-p_{0}\right|$. Then there exists a small neighborhood $V \subset \Omega$ of $p_{0}$ such that the characteristic curve through a point in $V \backslash\left\{p_{0}\right\}$ reaches $p_{0}$ (towards the $-N^{\perp}$ direction) in finite unit-speed parameter.

Proof. Write $p-p_{0}=(\triangle x, \Delta y)=(r \cos \varphi, r \sin \varphi)$ in polar coordinates. At $p$, we express

$$
N^{\perp}=\alpha(\cos \varphi, \sin \varphi)+\beta(-\sin \varphi, \cos \varphi)
$$

where

$$
\alpha=N^{\perp} \cdot(\cos \varphi, \sin \varphi), \quad \beta=N^{\perp} \cdot(-\sin \varphi, \cos \varphi) .
$$

Noting that $u_{x x}=u_{x y}=u_{y y}=0$ at $p_{0}$ by Lemma 3.8, we obtain that

$$
u_{y}+x=\Delta x+o(r), \quad-u_{x}+y=\Delta y+o(r)
$$

near $p_{0}$ by (3.11) and (3.12). It follows that $D=\left[(\triangle x)^{2}+(\triangle y)^{2}\right]^{1 / 2}+o(r)$ $=r+o(r)$ near $p_{0}$. From this and (3.17), we can estimate $N^{\perp}=\left(\frac{\Delta x}{r}+o(1)\right.$, $\left.\frac{\Delta y}{r}+o(1)\right)$. Substituting this and $(\cos \varphi, \sin \varphi)=\left(\frac{\Delta x}{r}, \frac{\Delta y}{r}\right)$ in (3.16) gives

$$
\alpha=\frac{(\triangle x)^{2}+(\triangle y)^{2}}{r^{2}}+o(1)=1+o(1)
$$


Now let $(x(s), y(s))$ describe a characteristic curve in the $-N^{\perp}$ direction, i.e., $\frac{d(x(s), y(s))}{d s}=-N^{\perp}$. We compute

$$
\begin{aligned}
\frac{d(x(s), y(s))}{d s} & =\frac{d(r(s) \cos \varphi(s), r(s) \sin \varphi(s))}{d s} \\
& =\frac{d r}{d s}(\cos \varphi, \sin \varphi)+r \frac{d \varphi}{d s}(-\sin \varphi, \cos \varphi) .
\end{aligned}
$$

Comparing with (3.15), we obtain

$$
\frac{d r}{d s}=-\alpha, \quad r \frac{d \varphi}{d s}=-\beta .
$$

Observe that $\alpha$ tends to 1 as $r$ goes to 0 by (3.18) (hence $\beta=o(1)$ since $\alpha^{2}+\beta^{2}=1$ ). So from (3.19) we can find a small neighborhood $V$ of $p_{0}$ so that the distance between $p_{0}$ and the characteristic curve $\Gamma$ through a point $p_{1} \in V \backslash\left\{p_{0}\right\}$ is decreasing towards the $-N^{\perp}$ direction. Let $s$ denote a unit-speed parameter of $\Gamma$ and $p_{1}=\Gamma\left(s_{1}\right)$. Then from the following formula

$$
r(s)-r\left(s_{1}\right)=\int_{s_{1}}^{s} \frac{d r}{d s} d s=\int_{s_{1}}^{s}(-\alpha) d s,
$$

we learn that $r(s)$ reaches 0 for a finite $s$.

Let $B_{r}\left(p_{0}\right)=\left\{p \in \Omega|| p-p_{0} \mid<r\right\}$. Define $H_{M}(r)=\max _{p \in \partial B_{r}\left(p_{0}\right)}|H(p)|$.

Theorem 3.10. Let $u \in C^{2}(\Omega)$. Suppose $|H|=o\left(\frac{1}{r}\right)$ (little "o") near an isolated singular point $p_{0} \in \Omega$ where $r(p)=\left|p-p_{0}\right|$. Moreover, suppose there is $r_{0}>0$ such that

$$
\int_{0}^{r_{0}} H_{M}(r) d r<\infty
$$

Then for any unit tangent vector $v$ at $p_{0}$, there exists a unique characteristic curve $\Gamma$ touching $p_{0}$ (i.e. $p_{0} \in \bar{\Gamma}$, the closure of $\Gamma$ ) such that $N^{\perp}(u)\left(p_{0}^{+}\right)$, the limit of $N^{\perp}(u)$ at $p_{0}$ along $\Gamma$, equals $v$. Moreover, there exists a neighborhood $V$ of $p_{0}$ such that $V \backslash\left\{p_{0}\right\}$ is contained in the union of all such $\Gamma$ 's.

Proof. Take $\delta>0$ small enough so that all characteristic curves through points on $\partial B_{\delta}\left(p_{0}\right)$ reach $p_{0}$ in finite unit-speed parameter in view of Lemma 3.9. Let $\Gamma$ denote the characteristic curve through a point $p_{1} \in \partial B_{\delta}\left(p_{0}\right)$. Let $\left(s_{0}, s_{1}\right]$ be the interval of unit-speed paramater describing points of $\Gamma$ between $p_{0}$ and $p_{1}$. Take a sequence of points $p_{j} \in \Gamma \rightarrow p_{0}$ with parameter $s_{j} \rightarrow s_{0}$. We compute

$$
\theta\left(p_{j}\right)-\theta\left(p_{k}\right)=\int_{s_{k}}^{s_{j}} \frac{d \theta}{d s} d s=-\int_{s_{k}}^{s_{j}} H d s
$$

by (2.23) (recall that $\theta$ is defined by $N=N(u)=(\cos \theta, \sin \theta)$ ). It follows from $\frac{d r}{d s} \rightarrow-1$ as $s \rightarrow s_{0}$ or $r \rightarrow 0$ and (3.20) that

$$
\left|\theta\left(p_{j}\right)-\theta\left(p_{k}\right)\right| \leq \int_{r_{k}}^{r_{j}} H_{M}(r)\left|\frac{d s}{d r}\right| d r \leq 2 \int_{r_{k}}^{r_{j}} H_{M}(r) d r \rightarrow 0
$$


as $p_{j}, p_{k} \rightarrow p_{0}$. This means that $\left\{\theta\left(p_{j}\right)\right\}$ is a Cauchy sequence. Therefore it converges to some number, denoted as $\theta\left(p_{0} ; p_{1}\right)$. Define a map $\Psi: \partial B_{\delta}\left(p_{0}\right)$ $\rightarrow S^{1}$ by $\Psi(q)=\theta\left(p_{0} ; q\right)$. We claim that $\Psi$ is a homeomorphism. Take a sequence of points $q_{j} \in \partial B_{\delta}\left(p_{0}\right)$ converging to $\hat{q}$. We want to show that $\theta\left(p_{0} ; q_{j}\right)$ converges to $\theta\left(p_{0} ; \hat{q}\right)$. Without loss of generality, we may assume all $q_{j}^{\prime} s$ are sitting on one side of $\hat{q}$ so that $\varphi\left(q_{1}\right)>\varphi\left(q_{2}\right)>\ldots>\varphi(\hat{q})$ where $\varphi$ is the angle in polar coordinates centered at $p_{0}$ ranging in $[0,2 \pi)$. Observe that

$$
\begin{gathered}
\theta\left(p_{0} ; q_{j}\right) \geq \theta\left(p_{0} ; q_{j+1}\right) \geq \ldots \geq \theta\left(p_{0} ; \hat{q}\right) \\
\quad(\text { letting } \theta \text { take values in }[0,2 \pi))
\end{gathered}
$$

for $j$ large since two distinct characteristic curves can not intersect in $B_{\delta}\left(p_{0}\right) \backslash\left\{p_{0}\right\}$. Let $\hat{\theta}$ be the limit of $\theta\left(p_{0} ; q_{j}\right)$ as $j \rightarrow \infty$. Now suppose $\hat{\theta} \neq \theta\left(p_{0} ; \hat{q}\right)$ (hence $\left.\hat{\theta}>\theta\left(p_{0} ; \hat{q}\right)\right)$. Let $\Gamma_{j}\left(\hat{\Gamma}\right.$, resp.) denote the characteristic curve connecting $q_{j}$ $\left(\hat{q}\right.$, resp.) and $p_{0}$. Then we can find two rays emitting from $p_{0}$ with angle smaller than $\hat{\theta}-\theta\left(p_{0} ; \hat{q}\right)$ and a small positive $\hat{\delta}<\delta$ so that $\Gamma_{j}$ and $\hat{\Gamma}$ do not meet a fan-shaped region $\hat{\Omega}$ surrounded by these two rays and $\partial B_{\hat{\delta}}\left(p_{0}\right)$ for $j$ large. Take a point $\check{p} \in \hat{\Omega}$. Consider the characteristic curve $\check{\Gamma}$ through $\check{p}$. Then $\check{\Gamma}$ must intersect $\partial B_{\delta}\left(p_{0}\right)$ at a point $\check{q}$ while reaching $p_{0}$ with $\theta=\theta\left(p_{0} ; \check{q}\right)$. Since $\check{\Gamma}$ does not intersect with any $\Gamma_{j}$, we have $\theta\left(p_{0} ; q_{j}\right)>\theta\left(p_{0} ; \check{q}\right)$ for large $j$. On the other hand, $\breve{q}$ must coincide with $\hat{q}$ for the same reason. So $\check{\Gamma}=\hat{\Gamma}$, an obvious contradiction. Thus $\hat{\theta}=\theta\left(p_{0} ; \hat{q}\right)$. We have proved the continuity of $\Psi$.

Next we claim that $\Psi$ is surjective. If not, $S^{1} \backslash \Psi\left(\partial B_{\delta}\left(p_{0}\right)\right)$ is a nonempty open set. Then by a similar fan-shaped region argument as shown above, we can reach a contradiction. Let $\Gamma_{1}, \Gamma_{2}$ be two characteristic curves through $q_{1}$, $q_{2} \in \partial B_{\delta}\left(p_{0}\right)$ touching $p_{0}$ with $\theta\left(p_{0} ; q_{1}\right)=\theta\left(p_{0} ; q_{2}\right)$. We want to show that $q_{1}$ $=q_{2}$. Suppose $q_{1} \neq q_{2}$. So $\Gamma_{1}$ and $\Gamma_{2}$ are distinct (with empty intersection in $\left.B_{\delta}\left(p_{0}\right) \backslash\left\{p_{0}\right\}\right)$ and tangent at $p_{0}$. Let $\Omega_{r}$ denote the smaller domain, surrounded by $\Gamma_{1}, \Gamma_{2}$, and $\partial B_{r}\left(p_{0}\right)$ for small $r>0$. Then $\Omega_{r}$ is contained in a fan-shaped region with vertex $p_{0}$ and angle $\theta_{r}$ such that $\theta_{r} \rightarrow 0$ as $r \rightarrow 0$. Let $\Gamma_{r} \equiv$ $\partial \Omega_{r} \cap \partial B_{r}\left(p_{0}\right)$. It follows that

$$
\left|\Gamma_{r}\right| \leq r \theta_{r}
$$

where $\left|\Gamma_{r}\right|$ denotes the length of the arc $\Gamma_{r}$. Since $N^{\perp}(u)$ is perpendicular to the unit outward normal $v(= \pm N(u))$ on $\Gamma_{1}$ and $\Gamma_{2}$, we obtain

$$
g(r) \equiv \oint_{\partial \Omega_{r}}\left(u_{y}+x,-u_{x}+y\right) \cdot v d s=\int_{\Gamma_{r}}\left(u_{y}+x,-u_{x}+y\right) \cdot v d s .
$$

Observing that $\left(u_{y}+x,-u_{x}+y\right)=p-p_{0}+o(r)$ by (3.17) and $v=\frac{p-p_{0}}{r}$ on $\Gamma_{r}$, we deduce from (3.23) that

$$
g(r)=[r+o(r)]\left|\Gamma_{r}\right| .
$$


On the other hand, the divergence theorem tells us that

$$
\begin{aligned}
g(r) & =\iint_{\Omega_{r}}\left[\left(u_{y}+x\right)_{x}+\left(-u_{x}+y\right)_{y}\right] d x \wedge d y \\
& =2 \iint_{\Omega_{r}} d x \wedge d y=2 \int_{0}^{r}\left|\Gamma_{\tau}\right| d \tau .
\end{aligned}
$$

It follows from (3.25) that $g^{\prime}(r)=2\left|\Gamma_{r}\right|$. Comparing this with (3.24), we obtain $\frac{g^{\prime}(r)}{g(r)}=\frac{2}{r}+o\left(\frac{1}{r}\right)$. Therefore $g(r)=c r^{2}+o\left(r^{2}\right)$ for some constant $c>0$. However, inserting (3.22) into (3.24) shows that $g(r)=o\left(r^{2}\right)$, i.e., $\frac{g(r)}{r^{2}} \rightarrow 0$ as $r \rightarrow 0$. We have reached a contradiction. So $q_{1}=q_{2}$ and hence $\Psi^{r^{2}}$ is injective. Next we will show that $\Psi^{-1}$ is continuous. Suppose this is not true. Then we can find a sequence of $q_{j} \in \partial B_{\delta}\left(p_{0}\right)$ converging to $\check{q} \neq \hat{q}$ while $\theta\left(p_{0} ; q_{j}\right)$ converges to $\theta\left(p_{0} ; \hat{q}\right)$ (may assume monotonicity (3.21) or reverse order for large $j$ ). Take a point $\bar{q} \in \partial B_{\delta}\left(p_{0}\right), \bar{q} \neq \check{q}$ and $\bar{q} \neq \hat{q}$, such that $\theta\left(p_{0} ; q_{j}\right) \geq \theta\left(p_{0} ; \bar{q}\right) \geq$ $\theta\left(p_{0} ; \hat{q}\right)$ for all large $j$. Since $\lim \theta\left(p_{0} ; q_{j}\right)=\theta\left(p_{0} ; \hat{q}\right)$, we must have $\theta\left(p_{0} ; \bar{q}\right)$ $=\theta\left(p_{0} ; \hat{q}\right)$ contradicting the injectivity of $\Psi$. Altogether we have shown that $\Psi$ is a homeomorphism. The theorem follows from this fact.

Proof of Corollary 3.6. Let $N^{\perp}\left(p_{0}^{+}\right)$denote $N^{\perp}(u)\left(p_{0}^{+}\right)$for simplicity. First we claim that $N^{\perp}\left(p_{0}^{+}\right)$can not be tangent to $\Gamma_{s}$ at $p_{0}$. If yes, we have $N^{\perp}\left(p_{0}^{+}\right)\left(U\left(p_{0}\right)-U^{T}\left(p_{0}\right)\right)=0$ by Lemma 3.7 (1), (2). However, $U-U^{T}=$ $\left(\begin{array}{cc}0 & -2 \\ 2 & 0\end{array}\right)$. It follows that $N^{\perp}\left(p_{0}^{+}\right)=0$, a contradiction. So $N^{\perp}\left(p_{0}^{+}\right)$is transversal to $\Gamma_{s}$, and $N^{\perp}\left(p_{0}^{+}\right) U^{T}\left(p_{0}\right) \neq 0$. In fact, we have

$$
\left|N^{\perp}\left(p_{0}^{+}\right) U^{T}\left(p_{0}\right)\right|=2
$$

by noting that the unit-length vector $N^{\perp}\left(p_{0}^{+}\right)$is proportional to $\left(u_{x y}+1,-u_{x x}\right)$ (if $\neq 0$ ) and $\left(u_{y y},-u_{x y}+1\right)$ (if $\neq 0$ ) from the proof of Lemma 3.7 or Proposition 3.5. Now suppose $\Gamma_{+}$and $\Gamma_{+}^{\prime}$ are two distinct (never intersect in $B_{\epsilon}\left(p_{0}\right) \backslash\left\{p_{0}\right\}$ for some small $\left.\epsilon\right)$ characteristic curves contained in $B^{+}$touching $p_{0}$ (hence with the same tangent vector $N^{\perp}\left(p_{0}^{+}\right)$at $\left.p_{0}\right)$. Let $\Omega_{r}$ denote the domain, surrounded by $\Gamma_{+}, \Gamma_{+}^{\prime}$, and $\partial B_{r}\left(p_{0}\right)$ for $0<r<\epsilon$. Then $\Omega_{r}$ is contained in a fan-shaped region with vertex $p_{0}$ and angle $\theta_{r}$ such that $\theta_{r} \rightarrow 0$ as $r \rightarrow 0$. Let $\Gamma_{r} \equiv \partial \Omega_{r} \cap \partial B_{r}\left(p_{0}\right)$. Then (3.22) holds.

On the other hand, $\left(u_{x}-y, u_{y}+x\right)=(\Delta x, \triangle y) U^{T}\left(p_{0}\right)+o\left(r^{2}\right)$ by (3.11) and (3.12) while $(\Delta x, \Delta y)=r N^{\perp}\left(p_{0}^{+}\right)+o(r)$ and $v=(\Delta x, \Delta y) r^{-1}$ on $\Gamma_{r}$ tends to $N^{\perp}\left(p_{0}^{+}\right)$as $r \rightarrow 0$. So from (3.23) we compute

$$
\begin{aligned}
g(r) & =\int_{\Gamma_{r}}\left(u_{y}+x,-u_{x}+y\right) \cdot v d s \\
& =\int_{\Gamma_{r}} D N^{\perp} \cdot v d s \\
& =(2 r+o(r))\left|\Gamma_{r}\right| .
\end{aligned}
$$


Here we have used (3.26) in the last equality. Now a similar argument as in the proof of Theorem 3.10 by comparing (3.25) with (3.27) gives $g(r)=c r+o(r)$ for a positive constant $c$. However, substituting (3.22) into (3.27) shows that $g(r)=o\left(r^{2}\right)$. We have reached a contradiction. Therefore $\Gamma_{+}$must coincide with $\Gamma_{+}^{\prime}$. Similarly we have a unique characteristic curve $\Gamma_{-} \subset B^{-}$touching $\Gamma_{s}$ also at $p_{0}$ so that $\Gamma_{-} \cup\left\{p_{0}\right\} \cup \Gamma_{+}$forms a $C^{1}$ smooth curve in $B_{\epsilon}\left(p_{0}\right)$.

The line integral in (3.23) has a geometric interpretation. Recall that the standard contact form in the Heisenberg group $H_{1}$ is $\Theta_{0}=d z+x d y-y d x$. Let $\tilde{u}$ denote the map: $(x, y) \rightarrow(x, y, u(x, y))$. It is easy to see that $\tilde{u}^{*} \Theta_{0}=\left(u_{y}+x\right) d y$ $-\left(y-u_{x}\right) d x$. Now it is clear that the line integral in (3.23) is exactly the line integral of $\tilde{u}^{*} \Theta_{0}$. Note that $\tilde{u}^{*} \Theta_{0}$ vanishes along any characteristic curve. If we remove the condition (3.20) in Theorem 3.10, $\theta\left(p_{0} ; p_{1}\right)$ will not exist as shown in the following example.

Example. Let $p_{0}=(0,0)$. Let $u=-\frac{r^{2}}{\log r^{2}}\left(=0\right.$ at $\left.p_{0}\right)$ where $r^{2}=x^{2}+y^{2}$. Write $u=f\left(r^{2}\right)$. A direct computation shows that

$$
\begin{aligned}
u_{x} & =2 x f^{\prime}\left(r^{2}\right), \quad u_{y}=2 y f^{\prime}\left(r^{2}\right), \\
D & =r \sqrt{1+4\left(f^{\prime}\right)^{2}}, \\
u_{x x} & =2 f^{\prime}+4 x^{2} f^{\prime \prime}, u_{x y}=4 x y f^{\prime \prime}, u_{y y}=2 f^{\prime}+4 y^{2} f^{\prime \prime} .
\end{aligned}
$$

It is easy to see that $p_{0}$ is an isolated singularity (for a general $f$ ). Also $u$ is $C^{2}$ at $p_{0}$ and $u_{x x}=u_{x y}=u_{y y}=0$ at $p_{0}$ (for $f(t)=-\frac{t}{\log t}, t=r^{2}$ ). Therefore $d\left(N^{\perp} D\right)_{p_{0}}$ is the identity transformation and the index of $N^{\perp} D$ is +1 . Noting that $(-\sin \varphi, \cos \varphi)=(-y, x) r^{-1}$, we compute $\beta=D^{-1} r^{-1}\left(u_{y},-u_{x}\right)$. $(-y, x)=-2 f^{\prime} / \sqrt{1+4\left(f^{\prime}\right)^{2}}$ by (3.16), (3.28), and (3.29). Therefore along a characteristic curve reaching $p_{0}$ in the $-N^{\perp}$ direction, we can estimate

$$
\begin{aligned}
\frac{d \varphi}{d s} & =-\frac{1}{r} \beta(\text { by (3.19)) } \\
& =\frac{2 f^{\prime}}{r \sqrt{1+4\left(f^{\prime}\right)^{2}}} \approx \frac{2}{-r \log r^{2}}
\end{aligned}
$$

as $r \rightarrow 0$ for $f(t)=-\frac{t}{\log t}, t=r^{2}$. Since $\frac{d r}{d s} \rightarrow-1$ by (3.18) and

$$
\int_{0}^{r_{1}} \frac{2}{-r \log r^{2}} d r=\infty
$$

we conclude from (3.31) that $\varphi \rightarrow \infty$, hence $\theta \rightarrow \infty$ as the point on the characteristic curve approaches $p_{0}$. (Observing that $\alpha \rightarrow 1$ and $\beta \rightarrow 0$ in (3.15) as $r \rightarrow 0$, we have the limit of $\theta$ equal to the limit of $\varphi$ plus $\pi / 2$ if one of the limits exists, hence another limit exists too.) 
Next substituting (3.28) and (3.30) into (3.10) gives

$$
P(u)=2 r^{2}\left[f^{\prime}+4\left(f^{\prime}\right)^{3}+2 r^{2} f^{\prime \prime}\right] .
$$

By (2.10), (3.29), and (3.32), we obtain the p-mean curvature

$$
H=\frac{2\left(f^{\prime}+4\left(f^{\prime}\right)^{3}+2 r^{2} f^{\prime \prime}\right)}{r\left(1+4\left(f^{\prime}\right)^{2}\right)^{3 / 2}} .
$$

For $f(t)=-\frac{t}{\log t}, t=r^{2}, f^{\prime} \approx-\frac{1}{\log t}, 2 r^{2} f^{\prime \prime} \approx \frac{2}{(\log t)^{2}}$ near $r=0$. Inserting these estimates into (3.33) gives $H \approx-\frac{1}{r \log r}$. It is now a straightforward computation to verify that $H=o\left(\frac{1}{r}\right)$ and the integral in (3.20) for such an $H$ diverges.

\section{A Bernstein-type theorem and properly embedded p-minimal surfaces}

Recall that the characteristic curves for a p-minimal surface $\Sigma$ in a pseudohermitian 3-manifold $M$ are Legendrian geodesics in $M$ by (2.1). For $M=H_{1}$, we have

Proposition 4.1. The Legendrian geodesics in $H_{1}$, identified with $\mathbb{R}^{3}$, with respect to $\nabla^{\text {p.h. }}$ are straight lines.

Proof. Write a unit Legendrian vector field $e_{1}=f \hat{e}_{1}+g \hat{e}_{2}$ with $f^{2}+g^{2}=1$. Since $\nabla^{p \cdot h \cdot \hat{e}_{1}}=\nabla^{p \cdot h \cdot \hat{e}_{2}}=0$, the geodesic equation $\nabla_{e_{1}}^{p . h} \cdot e_{1}=0$ implies that $e_{1} f=e_{1} g=0$. This means that $f=c_{1}, g=c_{2}$ for some constants $c_{1}, c_{2}$ along a geodesic $\Gamma$ (integral curve) of $e_{1}$. We compute

$$
\begin{aligned}
e_{1} & =c_{1} \hat{e}_{1}+c_{2} \hat{e}_{2}=c_{1}\left(\partial_{x}+y \partial_{z}\right)+c_{2}\left(\partial_{y}-x \partial_{z}\right) \\
& =c_{1} \partial_{x}+c_{2} \partial_{y}+\left(c_{1} y-c_{2} x\right) \partial_{z} .
\end{aligned}
$$

So $\Gamma$ is described by the following system of ordinary differential equations:

$$
\begin{aligned}
& \frac{d x}{d s}=c_{1}, \\
& \frac{d y}{d s}=c_{2}, \\
& \frac{d z}{d s}=c_{1} y-c_{2} x .
\end{aligned}
$$

By (4.2a), (4.2b) we get $x=c_{1} s+d_{1}, y=c_{2} s+d_{2}$ for some constants $d_{1}, d_{2}$. Substituting into (4.2c) gives $z=\left(c_{1} d_{2}-c_{2} d_{1}\right) s+d_{3}$ for some constant $d_{3}$. So $\Gamma$ is a straight line in $\mathbb{R}^{3}$. 
Corollary 4.2. The characteristic curves of a p-minimal surface in $H_{1}$ are straight lines or line segments. In particular, a characteristic curve (line) of a p-minimal surface in $H_{1}$ through a point $q$ is contained in the contact plane through $q$.

Recall that $F_{a, b} \equiv a\left(u_{x}-y\right)+b\left(u_{y}+x\right)$ for real constants $a, b$ with $a^{2}+b^{2}=1$.

Lemma 4.3. Suppose $u \in C^{2}$ defines a p-minimal graph near $p \in S(u)$, an isolated singular point. Then $F_{a, b}=0$, for $a, b \in R, a^{2}+b^{2}=1$, define all straight line segments passing through $p$ which are all characteristic curves in a neighborhood of $p$ with $p$ deleted.

Proof. First we claim $\nabla F_{a, b}(p) \neq 0$ for all $(a, b)$ with $a^{2}+b^{2}=1$. If not, there exists $\left(a_{0}, b_{0}\right)$ such that $\nabla F_{a_{0}, b_{0}}(p)=0$. So $\operatorname{det} U(p)=0$ (see the paragraph after (3.1)). It follows from the proof of Theorem B that there is a small neighborhood of $p$ which intersects with $S(u)$ in exactly a $C^{1}$ smooth curve through $p$. This contradicts $p$ being an isolated singular point. We have shown that $\nabla F_{a, b}(p) \neq 0$ for all $(a, b)$ with $a^{2}+b^{2}=1$. Therefore $F_{a, b}=0$ defines a $C^{1}$ smooth curve through $p$ for all $(a, b)$.

In a neighborhood of $p$ with $p$ deleted, we observe that $F_{a, b} D^{-1} \equiv$ $\sin \theta_{0} \cos \theta-\cos \theta_{0} \sin \theta$. Here we write $a=\sin \theta_{0}, b=-\cos \theta_{0}$. Recall that $\left(u_{x}-y\right) D^{-1}=\cos \theta,\left(u_{y}+x\right) D^{-1}=\sin \theta$ (see Section 2). So $\theta=\theta_{0}$ on $\left\{F_{a, b}=0\right\}$, and hence by $(2.18 \mathrm{~b}) N^{\perp}=(\sin \theta,-\cos \theta)=\left(\sin \theta_{0},-\cos \theta_{0}\right)$ is a constant unit vector field along $\left\{F_{a, b}=0\right\}$. On the other hand, $\nabla\left(F_{a, b} D^{-1}\right)=$ $(-a \sin \theta+b \cos \theta) \nabla \theta$ is parallel to $N=(\cos \theta, \sin \theta)$ since $N^{\perp} \cdot \nabla \theta=0$ is our equation. It follows that $\left\{F_{a, b}=0\right\}$ is a straight line segment and an integral curve of $N^{\perp}$ in a $p$-deleted neighborhood.

We remark that Lemma 4.3 provides a more precise description of Theorem 3.10 in the case of $H=0$.

Since the characteristic curves are straight lines, we will often call them characteristic lines (line segments). From Corollary 3.6, we know that a characteristic line keeps straight after it goes through a singular curve. Note that two characteristic line segments $\Gamma_{1}, \Gamma_{2}$ can not touch a singular curve at the same point $p_{0}$ unless they lie on a straight line by Proposition 3.5 (the limits of $N(u)$ at $p_{0}$ along $\Gamma_{1}, \Gamma_{2}$ must be either the same or different by a sign). We say a graph is entire if it is defined on the whole $x y$-plane.

Lemma 4.4. Suppose $u \in C^{2}$ defines an entire p-minimal graph. Then $S(u)$ contains no more than one isolated singular point.

Proof. Suppose we have two such points $p_{1}, p_{2} \in S(u)$. Then there exist two distinct straight lines passing through $p_{1}, p_{2}$, respectively and intersecting at a third point $q$ such that $q \notin S(u)$ in view of Theorem B and Corollary 3.6. From the proof of Lemma 4.3 and Corollary 3.6, these two straight lines are characteristic curves in the complement of $S(u)$, namely integral curves of $N^{\perp}(u)$. But then at $q, N^{\perp}(u)$ has two values, a contradiction.

On the other hand, remember that we can change the sign of $N^{\perp}(u)$ if necessary to make a $C^{0}$ characteristic (i.e. tangent to integral curves of $N^{\perp}(u)$ where 
$N^{\perp}(u)$ is defined) vector field $\check{N}^{\perp}(u)$ on the whole $x y$-plane except possibly one isolated singular point in view of Proposition 3.5. Moreover, we have a unique characteristic curve "going through" a point of a singular curve by Corollary 3.6. So we can conclude that the following result holds.

Lemma 4.5. Suppose $u \in C^{2}$ defines an entire p-minimal graph and $S(u)$ contains no isolated singular point. Then all integral curves (restrict to characteristic lines of $\left.N^{\perp}(u)\right)$ of $\check{N}^{\perp}(u)$ are parallel.

Proof of Theorem A. According to Lemma 4.4, we have the following two cases.

Case 1. $S(u)$ contains one isolated singular point.

In this case, we claim the solution $u$ is nothing but (1.1). Let $p_{0}$ be the singular point. Let $r, \vartheta$ denote the polar coordinates with center $p_{0}$. We can write $\pm \check{N}^{\perp}(u)=\frac{\partial}{\partial r}$ in view of Lemma 4.3. By (2.20) we have the equation

$$
u_{r r}=\frac{\partial^{2} u}{\partial r^{2}}=0
$$

defined on the whole $x y$-plane except $p_{0}$. It follows from (4.3) that $u=r f(\vartheta)+$ $g(\vartheta)$ for some $C^{2}$ functions $f, g$ in $\vartheta$. Since $u$ is continuous at $p_{0}=\left(x_{0}, y_{0}\right)$ (where $r=0), u\left(x_{0}, y_{0}\right)=g(\vartheta)$ for all $\vartheta$. So $g$ is a constant function, say $g=c$. Also $f(\vartheta)=f(\vartheta+2 \pi)$ implies that we can write $f(\vartheta)=\tilde{f}(\cos \vartheta, \sin \vartheta)$ where $\tilde{f}$ is $C^{2}$ in $\alpha=\cos \vartheta$ and $\beta=\sin \vartheta$. Compute $u_{x}=u_{r} r_{x}+u_{\vartheta} \vartheta_{x}=$ $\alpha \tilde{f}+\beta^{2} \tilde{f}_{\alpha}-\alpha \beta \tilde{f}_{\beta}$ in which $\tilde{f}_{\alpha}=\partial \tilde{f} / \partial \alpha, \tilde{f}_{\beta}=\partial \tilde{f} / \partial \beta$, etc. and we have used $\vartheta_{x}=-(\sin \vartheta) / r$. Similarly we obtain $u_{y}=\beta \tilde{f}+\alpha^{2} \tilde{f}_{\beta}-\alpha \beta \tilde{f}_{\alpha}$. Since $u_{x}$ and $u_{y}$ are continuous at $\left(x_{0}, y_{0}\right)$, we immediately have the following identities:

$$
\begin{array}{r}
\beta^{2} \tilde{f}_{\alpha}-\alpha \beta \tilde{f}_{\beta}+\alpha \tilde{f}=a \\
-\alpha \beta \tilde{f}_{\alpha}+\alpha^{2} \tilde{f}_{\beta}+\beta \tilde{f}=b
\end{array}
$$

for all $\alpha, \beta$. Here $a=u_{x}\left(x_{0}, y_{0}\right), b=u_{y}\left(x_{0}, y_{0}\right)$. Multiplying (4.4), (4.5) by $\alpha$, $\beta$, respectively and adding the resulting identities, we obtain $\left(\alpha^{2}+\beta^{2}\right) \tilde{f}=a \alpha+$ $b \beta$. It follows that $\tilde{f}=a \alpha+b \beta$ since $\alpha^{2}+\beta^{2}=1$. We have shown that $u(x, y)$ $=r(a \cos \vartheta+b \sin \vartheta)+c=a\left(x-x_{0}\right)+b\left(y-y_{0}\right)+c_{0}=a x+b y+\left(c-a x_{0}-b y_{0}\right)$ $=a x+b y+c$. (In fact $\left(x_{0}, y_{0}\right)=(-b, a)$ from the definition of a singular point and the plane $\left\{(x, y, u(x, y)\}\right.$ is just the contact plane passing through $\left(x_{0}, y_{0}\right)$.) We can also give a geometric proof for Case 1 as follows. Let $\xi_{0}$ denote the standard contact bundle over $H_{1}$ (see the Appendix). Let $\Sigma$ denote the pminimal surface defined by $u$. Observe that the union of all characteristic lines "going through" $p_{0}$, the isolated singular point, (together with $p_{0}$ ) constitutes the contact plane $\xi_{0}\left(p_{0}\right)$ in view of Corollary 4.2 and Lemma 4.3. It follows that $\xi_{0}\left(p_{0}\right) \subset \Sigma$. So $\Sigma=\xi_{0}\left(p_{0}\right)$, an entire plane, since $\Sigma$ is also an entire graph. We are done. 
Case 2. $S(u)$ contains no isolated singular point.

In this case we claim $u$ is nothing but (1.2). By Lemma 4.5 and Lemma 3.4 we can find a rotation $\tilde{x}=a x+b y, \tilde{y}=-b x+a y$ with $a^{2}+b^{2}=1$ such that

$$
\check{N}^{\perp}(u)= \pm \frac{\partial}{\partial \tilde{x}} .
$$

By (2.20) our equation reads $\tilde{u}_{\tilde{x} \tilde{x}}=0$ where $\tilde{u}(\tilde{x}, \tilde{y})=u(x, y)$. It follows that

$$
\tilde{u}=\tilde{x} \tilde{y}+g(\tilde{y}),
$$

for some $C^{2}$ smooth functions $f, g$. From (4.6) we know $N(u)=(0, \pm 1)$. By the definition of $N(u)$ we obtain $\tilde{u}_{\tilde{x}}-\tilde{y}=0$. So $f(\tilde{y})=\tilde{y}$. Substituting this into (4.7) gives $\tilde{u}=\tilde{x} \tilde{y}+g(\tilde{y})$, and hence $u=-a b x^{2}+\left(a^{2}-b^{2}\right) x y+a b y^{2}+$ $g(-b x+a y)$.

We remark that the singular curve in Case 2 is defined by $\tilde{x}=-g^{\prime}(\tilde{y}) / 2$, and this curve has only one connected component.

Next we will describe a general properly embedded p-minimal surface in $H_{1}$, which may not be a graph. According to Proposition 4.1, such a surface must be a properly embedded ruled surface with Legendrian (tangent to contact planes) rulings when we view $H_{1}$ as $\mathbb{R}^{3}$. We call a ruled surface with Legendrian rulings a Legendrian ruled surface. Conversely, we claim that a properly embedded Legendrian ruled surface is a properly embedded p-minimal surface. First observe that a straight line $L$ through $p_{0}=\left(x_{0}, y_{0}, z_{0}\right)$ pointing in a contact direction $c_{1} \hat{e}_{1}\left(p_{0}\right)+c_{2} \hat{e}_{2}\left(p_{0}\right), c_{1}^{2}+c_{2}^{2}=1$, is tangent to the contact plane everywhere. Here $\hat{e}_{1}\left(p_{0}\right)=\partial_{x}+y_{0} \partial_{z}$ or $\left(1,0, y_{0}\right)$ and $\hat{e}_{2}\left(p_{0}\right)=$ $\partial_{y}-x_{0} \partial_{z}$ or $\left(0,1,-x_{0}\right)$. In fact we can parametrize any point $p=(x, y, z)$ $\in L$ as follows:

$$
(x, y, z)=\left(x_{0}, y_{0}, z_{0}\right)+s\left[c_{1} \hat{e}_{1}\left(p_{0}\right)+c_{2} \hat{e}_{2}\left(p_{0}\right)\right]
$$

for some $s \in R$. The tangent vector at $p$ is just $c_{1} \hat{e}_{1}\left(p_{0}\right)+c_{2} \hat{e}_{2}\left(p_{0}\right)$ which exactly equals $c_{1} \hat{e}_{1}(p)+c_{2} \hat{e}_{2}(p)$ by a simple computation. So it is a vector in the contact plane at $p$. And $L$ is a Legendrian line. A Legendrian ruled surface is generated by such Legendrian lines with its characteristic field $e_{1}(p)$ $=c_{1} \hat{e}_{1}\left(p_{0}\right)+c_{2} \hat{e}_{2}\left(p_{0}\right)=c_{1} \hat{e}_{1}(p)+c_{2} \hat{e}_{2}(p)$ with $c_{1}, c_{2}$ being constant along the characteristic line (or line segment) through a nonsingular point $p$. It follows that $\nabla_{e_{1}}^{p . h .} e_{1}=c_{1} \nabla_{e_{1}}^{p . h} \cdot \hat{e}_{1}+c_{2} \nabla_{e_{1}}^{p . h .} \hat{e}_{2}=0$ since $\nabla^{p . h .} \hat{e}_{j}=0, j=1,2$. By (2.1) the p-mean curvature $H$ vanishes. So we have shown that a Legendrian ruled surface is a p-minimal surface. Also an immersed Legendrian ruled surface is the union of a family of curves of the form (4.8), and has the following expression:

$$
\left(x_{0}(\tau), y_{0}(\tau), z_{0}(\tau)\right)+s\left[\sin \theta(\tau)\left(1,0, y_{0}(\tau)\right)-\cos \theta(\tau)\left(0,1,-x_{0}(\tau)\right)\right] .
$$

Here $\left(x_{0}(\tau), y_{0}(\tau), z_{0}(\tau)\right)$ is a curve transverse to rulings, and we have written $c_{1}(\tau)=\sin \theta(\tau)$ and $c_{2}(\tau)=-\cos \theta(\tau)$. 
Example. In (4.9) we take $\gamma(\tau) \equiv\left(x_{0}(\tau), y_{0}(\tau), z_{0}(\tau)\right)=(\cos \tau$, $\sin \tau, 0)$ and $\theta(\tau)=\tau, 0 \leq \tau<2 \pi$. It is easy to see that $e_{1}(\tau)=(\sin \tau,-\cos \tau, 1)$ (note that $e_{1}$ is independent of $\left.s\right)$. Compute $e_{1}\left(\tau_{1}\right) \times e_{1}\left(\tau_{2}\right) \cdot\left(\gamma\left(\tau_{2}\right)-\gamma\left(\tau_{1}\right)\right)=$ $\left(\sin \tau_{2}-\sin \tau_{1}\right)^{2}+\left(\cos \tau_{2}-\cos \tau_{1}\right)^{2}$. So $e_{1}\left(\tau_{1}\right) \times e_{1}\left(\tau_{2}\right) \cdot\left(\gamma\left(\tau_{2}\right)-\gamma\left(\tau_{1}\right)\right)=0$ if and only if $\tau_{1}=\tau_{2}$. Now it is easy to see that this Legendrian ruled surface is embedded. Let us write down the $x, y, z$ components as follows:

$$
x(\tau, s)=\cos \tau+(\sin \tau) s, y(\tau, s)=\sin \tau-(\cos \tau) s, z(\tau, s)=s .
$$

So $\partial_{\tau}(x, y, z)=\left(\frac{\partial x}{\partial \tau}, \frac{\partial y}{\partial \tau}, \frac{\partial z}{\partial \tau}\right)=(-\sin \tau+(\cos \tau) s, \cos \tau+(\sin \tau) s, 0)$ and $\Theta_{0}\left(\partial_{\tau}(x, y, z)\right)=1+s^{2} \neq 0$. This means that the tangent vector $\left.\partial_{\tau}(x, y, z)\right)$ is not annihilated by the contact form $\Theta_{0}$. Therefore (4.10) defines a properly embedded p-minimal surface in $H_{1}$ with no singular points, which is not a vertical plane (i.e. having the equation $a x+b y=c$ ). In fact, eliminating the parameters $\tau$ and $s$ in (4.10) gives the equation $z^{2}=x^{2}+y^{2}-1$.

For a Legendrian ruled surface of graph type, we can have an alternative approach to show that it is p-minimal. Let $(x, y, u(x, y))$ describe such a Legendrian ruled surface. Suppose we can take $x$ as the parameter of the rulings (straight lines) for simplicity. Then $d^{2} / d x^{2}\{u(x, y(x))\}=0$ along a ruling. By the chain rule we have

$$
r+2 s a+t a^{2}=0
$$

where $a=\frac{d y}{d x}, r=u_{x x}, s=u_{x y}$, and $t=u_{y y}$. On the other hand, along a Legendrian line, we have the contact form $d z+x d y-y d x=0$. It follows that $\frac{d z}{d x}+x a-y=p+q a+x a-y=0$ where $p=u_{x}, q=u_{y}$. So $a=-\frac{p-y}{q+x}$ (if $q+x=0$, then $p-y=0$ ). Substituting this into (4.11) gives

$$
(q+x)^{2} r-2(q+x)(p-y) s+(p-y)^{2} t=0
$$

which is exactly (pMGE). We remark that a general ruled surface satisfies a third order partial differential equation (see page 225 in [Mo]. Solving (4.11) for " $a$ " in terms of $r, s, t$, and substituting the result into $d^{3} / d x^{3}\{u(x, y(x))\}$ $=0$ give such an equation).

\section{Comparison principle and uniqueness for the Dirichlet problem}

Let $\Omega$ be a domain (connected and proper open subset) in the $x y$-plane. Let $u, v: \Omega \rightarrow R$ be two $C^{1}$ functions. Recall the singular set $S(u)=\{(x, y) \in \Omega$ | $\left.u_{x}-y=0, u_{y}+x=0\right\}$ and $N(u)=[\nabla u+(-y, x)] D_{u}^{-1}$ where $D_{u}=$ $\sqrt{\left(u_{x}-y\right)^{2}+\left(u_{y}+x\right)^{2}}$ (e.g. see (2.15a)).

Lemma 5.1. Suppose we have the situation described above. Then the equality

$$
(\nabla u-\nabla v) \cdot(N(u)-N(v))=\frac{D_{u}+D_{v}}{2}|N(u)-N(v)|^{2}
$$

holds on $\Omega \backslash(S(u) \cup S(v))$. In particular, $(\nabla u-\nabla v) \cdot(N(u)-N(v))=0$ if and only if $N(u)=N(v)$. 
Proof. Let $\vec{\alpha}=\nabla u+(-y, x), \vec{\beta}=\nabla v+(-y, x)$. Noting that $N(u)=\frac{\vec{\alpha}}{|\vec{\alpha}|}$, $N(v)=\frac{\vec{\beta}}{|\vec{\beta}|}\left(D_{u}=|\vec{\alpha}|, D_{v}=|\vec{\beta}|\right)$, we compute

$$
\begin{aligned}
(\nabla u-\nabla v) \cdot(N(u)-N(v)) & =(\vec{\alpha}-\vec{\beta}) \cdot\left(\frac{\vec{\alpha}}{|\vec{\alpha}|}-\frac{\vec{\beta}}{|\vec{\beta}|}\right) \\
& =|\vec{\alpha}|+|\vec{\beta}|-\frac{\vec{\alpha} \cdot \vec{\beta}}{|\vec{\beta}|}-\frac{\vec{\alpha} \cdot \vec{\beta}}{|\vec{\alpha}|} \\
& =(|\vec{\alpha}|+|\vec{\beta}|)(1-\cos \vartheta)
\end{aligned}
$$

in which $\cos \vartheta=\frac{\vec{\alpha} \cdot \vec{\beta}}{|\vec{\alpha}||\vec{\beta}|}$. On the other hand, we compute $|N(u)-N(v)|^{2}=$ $\left|\frac{\vec{\alpha}}{|\vec{\alpha}|}-\frac{\vec{\beta}}{|\vec{\beta}|}\right|^{2}=2-2 \frac{\vec{\alpha} \cdot \vec{\beta}}{|\vec{\alpha}||\vec{\beta}|}=2(1-\cos \vartheta)$. Substituting this into the right-hand side of (5.2) gives (5.1).

Remark. For the prescribed mean curvature equation $\operatorname{div} T u=H$ in $R^{n}$ where $T u=\frac{\nabla u}{\sqrt{1+|\nabla u|^{2}}}$, we have the following structural inequality:

$$
\begin{aligned}
(\nabla u-\nabla v) \cdot(T u-T v) & \geq \frac{\sqrt{1+|\nabla u|^{2}}+\sqrt{1+|\nabla v|^{2}}}{2}|T u-T v|^{2} \\
& \geq|T u-T v|^{2} .
\end{aligned}
$$

The above inequality was discovered by Miklyukov [Mik], Hwang [Hw1], and Collin-Krust [CK] independently. Here we have adopted Hwang's method to prove Lemma 5.1 .

Next let $u \in C^{0}\left(\bar{\Omega} \backslash S_{1}\right), v \in C^{0}\left(\bar{\Omega} \backslash S_{2}\right)$, i.e., $u, v$ are not defined (may blow up) on sets $S_{1}, S_{2} \subset \Omega$, respectively. Let $S \equiv S_{1} \cup S_{2} \cup S(u) \cup S(v)$ where $S(u) \subset \Omega \backslash S_{1}, S(v) \subset \Omega \backslash S_{2}$.

Theorem 5.2. Suppose $\Omega$ is a bounded domain in the $x y$-plane and $\mathcal{H}_{1}(\bar{S})$, the 1-dimensional Hausdorff measure of $\bar{S}$, vanishes. Let $u \in C^{0}\left(\bar{\Omega} \backslash S_{1}\right) \cap C^{2}(\Omega \backslash S)$, $v \in C^{0}\left(\bar{\Omega} \backslash S_{2}\right) \cap C^{2}(\Omega \backslash S)$ such that

$$
\begin{aligned}
\operatorname{div} N(u) & \geq \operatorname{div} N(v) & & \text { in } \Omega \backslash S, \\
u & \leq v & & \text { on } \partial \Omega \backslash S .
\end{aligned}
$$

Then $N(u)=N(v)$ in $\Omega^{+} \backslash S$ where $\Omega^{+} \equiv\{p \in \Omega \mid u(p)-v(p)>0\}$.

Proof. First $\mathcal{H}_{1}(\bar{S})=0$ means that given any $\epsilon>0$, we can find countably many balls $B_{j, \epsilon}, j=1,2, \ldots$ such that $\bar{S} \subset \cup_{j=1}^{\infty} B_{j, \epsilon}$ and $\Sigma_{j=1}^{\infty}$ length $\left(\partial B_{j, \epsilon}\right)<\epsilon$ and we can arrange $\cup_{j=1}^{\infty} B_{j, \epsilon_{1}} \subset \cup_{j=1}^{\infty} B_{j, \epsilon_{2}}$ for $\epsilon_{1}<\epsilon_{2}$. Since $\bar{S}$ is compact, we can find finitely many $B_{j, \epsilon}$ 's, say $j=1,2, \ldots, n(\epsilon)$, still covering $\bar{S}$. Suppose $\Omega^{+} \neq \emptyset$. Then by Sard's theorem there exists a sequence of positive number $\delta_{i}$ converging to 0 as $i$ goes to infinity, such that $\Omega_{i}^{+} \equiv\left\{p \in \Omega \mid u(p)-v(p)>\delta_{i}\right\}$ 
$\neq \emptyset$ and $\partial \Omega_{i}^{+} \backslash S$ is $C^{2}$ smooth. Note that $\partial \Omega_{i}^{+} \cap \partial \Omega \subset S$ by (5.4). Now we consider

$$
I_{\epsilon}^{i}=\oint_{\partial\left(\Omega_{i}^{+} \backslash \cup_{j=1}^{n(\epsilon)} B_{j, \epsilon}\right)} \tan ^{-1}(u-v)(N(u)-N(v)) \cdot v d s
$$

where $v, s$ denote the outward unit normal vector and the arc length parameter, respectively. By the divergence theorem we have

$$
\begin{aligned}
I_{\epsilon}^{i}= & \iint_{\Omega_{i}^{+} \backslash \cup_{j=1}^{n(\epsilon)} B_{j, \epsilon}}\left\{\frac{1}{1+(u-v)^{2}}(\nabla u-\nabla v) \cdot(N(u)-N(v))\right. \\
& \left.+\tan ^{-1}(u-v) \operatorname{div}(N(u)-N(v))\right\} d x d y .
\end{aligned}
$$

Observe that the second term in the right hand side of (5.5) is nonnegative by (5.3). It follows from (5.1) and (5.5) that

$$
I_{\epsilon}^{i} \geq \iint_{\Omega_{i}^{+} \backslash \cup_{j=1}^{n(\epsilon)} B_{j, \epsilon}}\left\{\frac{1}{1+(u-v)^{2}}\left(\frac{D_{u}+D_{v}}{2}\right)|N(u)-N(v)|^{2}\right\} d x d y
$$

On the other hand, we can estimate

$$
\begin{aligned}
I_{\epsilon}^{i} \leq & \left(\tan ^{-1} \delta_{i}\right) \int_{\partial \Omega_{i}^{+} \backslash\left(\cup_{j=1}^{n(\epsilon)} B_{j, \epsilon}\right)}(N(u)-N(v)) \cdot v d s \\
& +\frac{\pi}{2} \cdot 2 \cdot \Sigma_{j=1}^{n(\epsilon)} \text { length }\left(\partial B_{j, \epsilon}\right) \leq \pi \cdot \Sigma_{j=1}^{\infty} \text { length }\left(\partial B_{j, \epsilon}\right)<\pi \epsilon
\end{aligned}
$$

by noting that $v=-\frac{\nabla(u-v)}{|\nabla(u-v)|}$ and hence $(N(u)-N(v)) \cdot v \leq 0$ by (5.1). If $N(u) \neq N(v)$ at some point $p$ in $\Omega^{+} \backslash \bar{S}$, then $N(u) \neq N(v)$ in an open neighborhood $V$ of $p$, contained in $\Omega_{i}^{+}$for all large $i$. Observe that the measure of $V \backslash \cup_{j=1}^{\infty} B_{j, \epsilon}$ is bounded from below by a positive constant independent of small enough $\epsilon$ and $i$. Thus from (5.6) $I_{\epsilon}^{i} \geq c$, a positive constant independent of small enough $\epsilon$ and large enough $i$. Letting $\epsilon$ go to 0 in (5.7) will give us a contradiction. So $N(u)=N(v)$ in $\Omega^{+} \backslash \bar{S}$ and hence in $\Omega^{+} \backslash S$ by continuity.

Remark. Theorem 5.2 is an analogue of Concus and Finn's general comparison principles for the prescribed mean curvature equation (cf. Theorem 6 in $[\mathrm{CF}]$ ). In [Hw2] Hwang invoked the " $\tan ^{-1}$ " technique to simplify the proof of [CF]. Here we have followed the idea of Hwang in [Hw2] to prove Theorem 5.2.

Lemma 5.3. Let $u, v \in C^{2}(\Omega) \cap C^{0}(\bar{\Omega})$ where $\Omega$ is a bounded domain in the $x y$-plane. Suppose $N(u)=N(v)$ in $\Omega \backslash(S(u) \cup S(v)), u=v$ on $\partial \Omega$. Then $u=v$ in $\Omega$. 
Proof. Suppose $u \neq v$ in $\Omega$. We may assume the set $\{p \in \Omega \mid u(p)>v(p)\} \neq \emptyset$ (otherwise, interchange $u$ and $v$ ). By Sard's theorem (e.g., [St], noting that $C^{2}$ is essential), there exists $\epsilon>0$ such that $\Omega_{\epsilon} \equiv\{p \in \Omega \mid u(p)-v(p)-\epsilon>0\}$ $\neq \emptyset$ and $\Gamma_{\epsilon} \equiv\{p \in \Omega \mid u(p)-v(p)=\epsilon\}$ is $C^{2}$ smooth. Note that $\bar{\Gamma}_{\epsilon} \cap \partial \Omega$ $=\emptyset$ since $u=v$ on $\partial \Omega$ by assumption. Also $\Gamma_{\epsilon}$ is closed and bounded, hence compact. Therefore $\Gamma_{\epsilon}$ is the union of (finitely-many) $C^{2}$ smooth loops. Choose one of them, and denote it as $\Gamma_{\epsilon}^{\prime}$. We claim

$$
\frac{d u}{d s}+x \frac{d y}{d s}-y \frac{d x}{d s}=0
$$

on $\Gamma_{\epsilon}^{\prime}$ where $s$ is a unit-speed parameter of $\Gamma_{\epsilon}^{\prime}$. For $p \in \Gamma_{\epsilon}^{\prime} \cap S(u)$, (5.8) holds by the definition of a singular point. For $p \in \Gamma_{\epsilon}^{\prime} \backslash(S(u) \cup S(v))$, we compute $N^{\perp}(u) u\left(N^{\perp}(u)\right.$ as an operator acting on $\left.u\right)$ as follows:

$$
\begin{aligned}
N^{\perp}(u) u & =N^{\perp}(u) \cdot \nabla u=D^{-1}\left\{(\nabla u)^{\perp}+(x, y)\right\} \cdot \nabla u \\
& =D^{-1}(x, y) \cdot \nabla u=D^{-1}(x, y) \cdot\{\nabla u+(-y, x)\} \\
& =(x, y) \cdot N(u) .
\end{aligned}
$$

Similarly we can show

$$
N^{\perp}(v) v=(x, y) \cdot N(v) .
$$

Since $N(u)=N(v)$, hence $N^{\perp}(u)=N^{\perp}(v)$ at $p$, we conclude that $N^{\perp}(u)(u-$ $v)=0$ at $p$ by (5.9) and (5.10). This means that $N^{\perp}(u)$ is tangent to $\Gamma_{\epsilon}^{\prime}$ at $p$. So (5.8) holds at $p$. For $p \in\left(\Gamma_{\epsilon}^{\prime} \backslash S(u)\right) \cap S(v), \vec{\beta}(\equiv \nabla v+(-y, x))=0$. We observe that $\vec{\alpha}(\equiv \nabla u+(-y, x))=\vec{\alpha}-\vec{\beta}=\nabla(u-v)$. This means that $N(u)$ $\left(\equiv \frac{\vec{\alpha}}{|\vec{\alpha}|}\right)$ is normal to $\Gamma_{\epsilon}^{\prime}$. So again $N^{\perp}(u)$ is tangent to $\Gamma_{\epsilon}^{\prime}$ at $p$. Thus (5.8) holds at $p$. We have shown that (5.8) holds for all $p \in \Gamma_{\epsilon}^{\prime}$. $\Gamma_{\epsilon}^{\prime}$ bounds a domain, denoted as $\Omega_{\epsilon}^{\prime}$. Now integrating (5.8) over $\Gamma_{\epsilon}^{\prime}$, we obtain that the area of $\Omega_{\epsilon}^{\prime}$ vanishes by the divergence theorem, an obvious contradiction.

Proof of Theorem C. It follows from Theorem 5.2 and Lemma 5.3.

We can generalize Lemma 5.1 in the following form. Let $\Omega$ be a domain in $R^{n}$. Let $u, v: \Omega \rightarrow R$ be two $C^{1}$ functions. Let $\vec{F}$ be a $C_{\rightarrow}^{0}$ vector field in $R^{n}$. Define $S(u, \vec{F})=\{p \in \Omega \mid \nabla u+\vec{F}=0$ at $p\}$ and $S(v, \vec{F})$ similarly.

Lemma 5.1'. On $\Omega \backslash[S(u, \vec{F}) \cup S(v, \vec{F})]$, we have the following identity:

$$
(\nabla u-\nabla v) \cdot\left(\frac{\vec{\alpha}}{|\vec{\alpha}|}-\frac{\vec{\beta}}{|\vec{\beta}|}\right)=\left(\frac{|\vec{\alpha}|+|\vec{\beta}|}{2}\right)\left|\frac{\vec{\alpha}}{|\vec{\alpha}|}-\frac{\vec{\beta}}{|\vec{\beta}|}\right|^{2}
$$

where $\vec{\alpha}=\nabla u+\vec{F}, \vec{\beta}=\nabla v+\vec{F}$.

In general a contact form $d z+\Sigma_{j=1}^{j=n} f_{j} d x^{j}$ in $R^{n+1}$ gives rise to an $\vec{F}=$ $\left(f_{1}, f_{2}, \ldots, f_{n}\right)$ such that $\nabla u+\vec{F}$ is the $R^{n}$-projection of the Legendrian normal 
to the graph $z=u\left(x^{1}, x^{2}, \ldots, x^{n}\right)$. To generalize Theorem 5.2 to a domain $\Omega$ in $R^{n}$ and replace $N(u), N(v)$ by $\frac{\vec{\alpha}}{|\vec{\alpha}|}, \frac{\vec{\beta}}{|\vec{\beta}|}$, we will use $S_{\vec{F}}=S_{1} \cup S_{2} \cup S(u, \vec{F}) \cup$ $S(v, \vec{F})$ instead of $S$.

Theorem 5.2'. Suppose $\Omega$ is a bounded domain in $R^{n}$ and $\mathcal{H}_{n-1}\left(\bar{S}_{\vec{F}}\right)=0$. Let $u \in C^{0}\left(\bar{\Omega} \backslash S_{1}\right) \cap C^{2}\left(\Omega \backslash S_{\vec{F}}\right), v \in C^{0}\left(\bar{\Omega} \backslash S_{2}\right) \cap C^{2}\left(\Omega \backslash S_{\vec{F}}\right)$, and $\vec{F} \in C^{1}(\Omega) \cap C^{0}(\bar{\Omega})$ such that

$$
\begin{aligned}
\operatorname{div}\left(\frac{\nabla u+\vec{F}}{|\nabla u+\vec{F}|}\right) & \geq \operatorname{div}\left(\frac{\nabla v+\vec{F}}{|\nabla v+\vec{F}|}\right) \quad \text { in } \quad \Omega \backslash S_{\vec{F}}, \\
u & \leq v \quad \text { on } \quad \partial \Omega \backslash S_{\vec{F}} .
\end{aligned}
$$

Then $\frac{\nabla u+\vec{F}}{|\nabla u+\vec{F}|}=\frac{\nabla v+\vec{F}}{|\nabla v+\vec{F}|}$ in $\Omega^{+} \backslash S_{\vec{F}}$ where $\Omega^{+} \equiv\{p \in \Omega \mid u(p)-v(p)>0\}$.

The proof of Lemma 5.1' (Theorem 5.2', respectively) is similar to that of Lemma 5.1 (Theorem 5.2, respectively). We can also generalize Lemma 5.3. Let $\Omega$ be a bounded domain in $R^{2 m}, m \geq 1$. Take two real functions $u, v$ $\in C^{2}(\Omega) \cap C^{0}(\bar{\Omega})$. Let $\vec{\alpha} \equiv \nabla u+\vec{F}$ where $\vec{F}=\left(f_{1}, f_{2}, \ldots, f_{2 m}\right)$ is a $C^{1}$ smooth vector field on $\Omega$. Define $\vec{F} * \equiv\left(f_{2},-f_{1}, f_{4},-f_{3}, \ldots, f_{2 m},-f_{2 m-1}\right)$. Denote $\frac{\vec{\alpha}}{|\vec{\alpha}|}$ by $N_{\vec{F}}(u)$ and the set $\{p \in \Omega \mid \vec{\alpha}=0\}$ by $S_{\vec{F}}(u)$.

Lemma 5.3'. Suppose we have the situation as described above. Suppose $N_{\vec{F}}(u)=$ $N_{\vec{F}}(v)$ in $\Omega \backslash\left(S_{\vec{F}}(u) \cup S_{\vec{F}}(v)\right), u=v$ on $\partial \Omega$, and $\operatorname{div} \vec{F}^{*}>0$ a.e. in $\Omega$. Then $u=v$ on $\bar{\Omega}$.

Proof. Suppose the conclusion is not true. We may assume the set $\{p \in \Omega \mid$ $u(p)>v(p)\} \neq \emptyset$. By Sard's theorem we can find a small $\epsilon>0$ such that $\Omega_{\epsilon} \equiv$ $\{p \in \Omega \mid u(p)-v(p)-\epsilon>0\} \neq \emptyset$ and $\Gamma_{\epsilon} \equiv\{p \in \Omega \mid u(p)-v(p)=\epsilon\}=\partial \Omega_{\epsilon}$ is $C^{2}$ smooth. Let $\vec{\alpha}^{*} \equiv\left(u_{y_{1}},-u_{x_{1}}, u_{y_{2}},-u_{x_{2}}, \ldots, u_{y_{m}},-u_{x_{m}}\right)+\vec{F} *$ (so that $\left.\vec{\alpha}^{*} \cdot \vec{\alpha}=0\right)$. Let $v=-\frac{\nabla(u-v)}{|\nabla(u-v)|}$ denote the outward unit normal to $\Gamma_{\epsilon}$. We claim

$$
\vec{\alpha}^{*} \cdot v=0
$$

on $\Gamma_{\epsilon}$. Note that $\vec{\alpha}=0$ if and only if $\vec{\alpha}^{*}=0$. So it is obvious that (5.11) holds for $p \in S_{\vec{F}}(u)$. Let $N_{\vec{F}}^{*}(u) \equiv \frac{\vec{\alpha}^{*}}{\left|\vec{\alpha}^{*}\right|}$ for $p \in \Omega \backslash S_{\vec{F}}(u)$. In case $p \in$ $\Gamma_{\epsilon} \backslash\left(S_{\vec{F}}(u) \cup S_{\vec{F}}(v)\right)$, we can generalize (5.9), (5.10) as follows:

$$
\begin{aligned}
N_{\vec{F}}^{*}(u) u & =\vec{F}^{*} \cdot N_{\vec{F}}(u), \\
N_{\vec{F}}^{*}(v) v & =\vec{F}^{*} \cdot N_{\vec{F}}(v) .
\end{aligned}
$$

Since $N_{\vec{F}}(u)=N_{\vec{F}}(v)$ by assumption, and hence $N_{\vec{F}}^{*}(u)=N_{\vec{F}}^{*}(v)$, we deduce from $\left(5.9^{\prime}\right)$ and $\left(5.10^{\prime}\right)$ that $N_{\vec{F}}^{*}(u)(u-v)=0$. So $N_{\vec{F}}^{*}(u)$ is tangent to $\Gamma_{\epsilon}$ (at $p$ ). This implies (5.11). For $p \in\left(\Gamma_{\epsilon} \backslash S_{\vec{F}}(u)\right) \cap S_{\vec{F}}(v)$, we still have (5.11) by a similar argument as in the proof of Lemma 5.3. We have proved (5.11) 
for all $p \in \Gamma_{\epsilon}$. Let $d A$ denote the volume element of $\Gamma_{\epsilon}$, induced from $R^{2 m}$. Now we compute

$$
\begin{aligned}
0 & \left.=\int_{\Gamma_{\epsilon}} \vec{\alpha}^{*} \cdot v d A \text { (by }(5.11)\right) \\
& =\int_{\Omega_{\epsilon}} \operatorname{div}\left(\vec{\alpha}^{*}\right) d \text { (volume) (by the divergence theorem) } \\
& =\int_{\Omega_{\epsilon}} \operatorname{div} \vec{F}^{*} d(\text { volume })>0
\end{aligned}
$$

by assumption. We have reached a contradiction.

We remark that the condition $\operatorname{div} \vec{F}^{*}>0$ is essential in Lemma 5.3'. Consider the case $\vec{F}=0$. Let $\Omega=B_{2}-\bar{B}_{1}$ where $B_{r}$ denotes the open ball of radius $r$. Let $u=f(r), v=g(r)$, and $f \neq g$ with the properties that $f(1)=g(1)$, $f(2)=g(2)$, and $f^{\prime}>0, g^{\prime}>0$ for $1 \leq r \leq 2$. It follows that $S_{\vec{F}}(u)=\{\nabla u=$ $0\}=\phi, S_{\vec{F}}(v)=\{\nabla u=0\}=\phi$, and $\nabla u=f^{\prime}(r) \nabla r, \nabla v=g^{\prime}(r) \nabla r$. Therefore we have

$$
\frac{\nabla u}{|\nabla u|}=\nabla r=\frac{\nabla v}{|\nabla v|}
$$

by noting that $|\nabla r|=1$. We have constructed a counterexample for the statement of Lemma $5.3^{\prime}$ if $\operatorname{div} \vec{F}^{*}>0$ is not satisfied.

For $\vec{F}=\left(-y_{1}, x_{1},-y_{2}, x_{2}, \ldots,-y_{m}, x_{m}\right)$, we have $\vec{F}^{*}=\left(x_{1}, y_{1}, x_{2}, y_{2}, \ldots\right.$, $\left.x_{m}, y_{m}\right)$. In this case, we can view the integrand in (5.12) geometrically: $\left(d \overline{x_{j} \wedge d} y_{j}\right.$ means deleting $d x_{j} \wedge d y_{j}$ )

$$
\begin{aligned}
\vec{\alpha}^{*} \cdot v d A & =\Sigma_{j=1}^{j=m}\left[\left(u_{y_{j}}+x_{j}\right) d y_{j}+\left(u_{x_{j}}-y_{j}\right) d x_{j}\right] \\
& \wedge d x_{1} \wedge d y_{1} \wedge \ldots \wedge d \widehat{x_{j} \wedge d} y_{j} \wedge \ldots \wedge d x_{m} \wedge d y_{m} \\
& =\left[d u+\Sigma_{j=1}^{j=m}\left(x_{j} d y_{j}-y_{j} d x_{j}\right)\right] \\
& \wedge\left(\Sigma_{j=1}^{j=m} d x_{1} \wedge d y_{1} \wedge \ldots \wedge d \widehat{x_{j} \wedge d} y_{j} \wedge \ldots \wedge d x_{m} \wedge d y_{m}\right) \\
& =\frac{1}{2^{m-1}(m-1) !} \Theta_{(m)} \wedge\left(d \Theta_{(m)}\right)^{m-1}
\end{aligned}
$$

Here $\Theta_{(m)} \equiv d u+\Sigma_{j=1}^{j=m}\left(x_{j} d y_{j}-y_{j} d x_{j}\right)$ is the standard contact form of the $2 m+1$-dimensional Heisenberg group, restricted to the hypersurface $\left\{\left(x_{1}, y_{1}\right.\right.$, $\left.x_{2}, y_{2}, \ldots, x_{m}, y_{m}, u\left(x_{1}, y_{1}, x_{2}, y_{2}, \ldots, x_{m}, y_{m}\right)\right\}$. Integrating the above form gives

$$
\begin{aligned}
\int_{\Gamma_{\epsilon}} \vec{\alpha}^{*} \cdot v d A & =\frac{1}{2^{m-1}(m-1) !} \int_{\partial \Omega_{\epsilon}} \Theta_{(m)} \wedge\left(d \Theta_{(m)}\right)^{m-1} \\
& =\frac{1}{2^{m-1}(m-1) !} \int_{\Omega_{\epsilon}}\left(d \Theta_{(m)}\right)^{m} \text { (by Stokes' theorem) } \\
& =\frac{1}{2^{m-1}(m-1) !} 2^{m} m ! \text { Volume }\left(\Omega_{\epsilon}\right)=2 m \text { Volume }\left(\Omega_{\epsilon}\right)
\end{aligned}
$$


In the last equality, we have used $d \Theta_{(m)}=2 \Sigma_{j=1}^{j=m} d x_{j} \wedge d y_{j}$ and hence $\left(d \Theta_{(m)}\right)^{m}=$ $2^{m} m ! d x_{1} \wedge d y_{1} \wedge \ldots \wedge d x_{m} \wedge d y_{m}$. Note that $\operatorname{div} \vec{F}^{*}=2 m$ in this case. In general, let $\Theta_{\vec{F}} \equiv d z+\Sigma_{j=1}^{j=2 m} f_{j} d x_{j}$ for $\vec{F}=\left(f_{1}, f_{2}, \ldots, f_{2 m}\right)$. We can easily compute

$$
\begin{aligned}
d \Theta_{\vec{F}} & \wedge \Sigma_{j=1}^{j=m}\left(d x_{1} \wedge d x_{2} \wedge \ldots \wedge d x_{2 j-1} \wedge d x_{2 j} \wedge \ldots \wedge d x_{2 m-1} \wedge d x_{2 m}\right) \\
& =\left(\operatorname{div} \vec{F}^{*}\right) d x_{1} \wedge d x_{2} \wedge \ldots \wedge d x_{2 m-1} \wedge d x_{2 m} .
\end{aligned}
$$

Note that in case $\Theta_{\vec{F}}=\Theta_{(m)}$ (with $u, x_{j}, y_{j}$ replaced by $z, x_{2 j-1}, x_{2 j}$, respectively), we have

$$
\begin{aligned}
& \left(d \Theta_{\vec{F}}\right)^{m-1}=\left(d \Theta_{(m)}\right)^{m-1} \\
& =2^{m-1}(m-1) ! \Sigma_{j=1}^{j=m}\left(d x_{1} \wedge d x_{2} \wedge \ldots \wedge d x_{2 j-1} \wedge d x_{2 j} \wedge \ldots \wedge d x_{2 m-1} \wedge d x_{2 m}\right) .
\end{aligned}
$$

We can generalize Theorem $\mathrm{C}$ to higher dimensions without the condition on the singular set. Let $N(u)=N_{\vec{F}}(u)$ and $S(u)=S_{\vec{F}}(u)$ for $\vec{F}=\left(-y_{1}, x_{1},-y_{2}, x_{2}, \ldots\right.$, $\left.-y_{m}, x_{m}\right)$.

Theorem $\mathbf{C}^{\prime}$. For a bounded domain $\Omega$ in $R^{2 m}, m \geq 2$, let $u, v \in C^{2}(\Omega) \cap C^{0}(\bar{\Omega})$ satisfy $\operatorname{div} N(u) \geq \operatorname{div} N(v)$ in $\Omega \backslash S$ and $u \leq v$ on $\partial \Omega$ where $S=S(u) \cup S(v)$. Then $u \leq v$ in $\Omega$.

First we observe the following size control of the singular set in general dimensions.

Lemma 5.4. Suppose $u \in C^{2}(\Omega)$ where $\Omega \subset R^{2 m}$. Then for any singular point $p \in S(u)$, there exists an open neighborhood $V \subset \Omega$ such that the $m$-dimensional Hausdorff measure of $S(u) \cap V$ is finite, and hence $\mathcal{H}_{2 m-1}(S(u))=0$ for $m \geq 2$.

Proof. Consider the map $G: p \in \Omega \rightarrow(\nabla u+\vec{F})(p)$. Computing the differential $d G$ of $G$ at a singular point $p$ (where $G(p)=0$ ), we obtain

$$
\left(u_{i j}\right)+\left(\begin{array}{cccccc}
0 & -1 & 0 & 0 & . & . \\
1 & 0 & 0 & 0 & . & . \\
0 & 0 & 0 & -1 & . & . \\
0 & 0 & 1 & 0 & . & . \\
. & . & . & . & . & . \\
. & . & . & . & . & .
\end{array}\right)
$$

in matrix form, where $\left(u_{i j}\right)$ is the Hessian. Let $(d G)^{T}$ denote the transpose of $d G$. It is easy to see that $2 m=\operatorname{rank}\left(d G-(d G)^{T}\right)$ since $u_{i j}=u_{j i}$. On the other hand, $\operatorname{rank}\left(d G-(d G)^{T}\right) \leq \operatorname{rank}(d G)+\operatorname{rank}(-d G)^{T}=2 \operatorname{rank}(d G)$. Therefore $\operatorname{rank}(d G) \geq m$. Hence the kernel of $d G$ has dimension $\leq m$. It follows by the implicit function theorem that there exists an open neighborhood $V$ of $p$ such that $G^{-1}(0) \cap V=S(u) \cap V$ is a submanifold of $V$, having dimension $\leq m$.

Proof of Theorem $C^{\prime}$. Observe that the condition $\mathcal{H}_{2 m-1}(\bar{S})=0$ (the dimension $n=2 \mathrm{~m}$ ) in the proof of Theorem 5.2 (and Theorem 5.2') can be replaced by the following condition: for any subdomain $O \subset \subset \Omega$, i.e., $\bar{O} \subset \Omega, \mathcal{H}_{2 m-1}(\bar{O} \cap \bar{S})=$ 0 . Since $S=S(u) \cup S(v)$ is closed in the compact set $\bar{O}, \bar{O} \cap \bar{S}=\bar{O} \cap S$. Now Theorem $C^{\prime}$ follows from Theorem 5.2' (with the size control condition on $\bar{S}$ replaced by the above-mentioned one), Lemma 5.3', and Lemma 5.4. 


\section{Second variation formula and area-minimizing property}

In this section we will derive the second variation formula for the p-area functional (2.5) and examine the p-mean curvature $H$ from the viewpoint of calibration geometry $([\mathrm{HL}])$. As a result we can prove the area-minimizing property for a p-minimal graph in $H_{1}$.

We follow the notation in Section 2. We assume the surface $\Sigma$ is pminimal. Let $f, g$ be functions with compact support away from the singular set and the boundary of $\Sigma$. Recall $T$ denotes the Reeb vector field of $\Theta$ (see Section 2 or the Appendix). We compute the second variation of (2.5) in the direction $V=f e_{2}+g T$ :

$$
\delta_{V}^{2} \int_{\Sigma} \Theta \wedge e^{1}=\int_{\Sigma} L_{V}^{2}\left(\Theta \wedge e^{1}\right)=\int_{\Sigma} i_{V} \circ d\left\{i_{V} \circ d\left(\Theta \wedge e^{1}\right)\right\} .
$$

Here we have used Stokes' theorem and the formula $L_{V}=i_{V} \circ d+d \circ i_{V}$ and $d^{2}=0$. By (2.7) and $H=\omega\left(e_{1}\right)$, we get

$$
d\left(\Theta \wedge e^{1}\right)=-H \Theta \wedge e^{1} \wedge e^{2} .
$$

We recall (see Section 2) to define a function $\alpha$ on $\Sigma \backslash S_{\Sigma}$ such that $\alpha e_{2}+T \in$ $T \Sigma$. Observe that $\left\{\alpha e_{2}+T, e_{1}\right\}$ is a basis of $T\left(\Sigma \backslash S_{\Sigma}\right)$. So on $\Sigma \backslash S_{\Sigma}$ we have

$$
e^{2} \wedge e^{1}=\alpha \Theta \wedge e^{1} .
$$

From (6.2) it is easy to see that $i_{V} \circ d\left(\Theta \wedge e^{1}\right)=g H e^{2} \wedge e^{1}-f H \Theta \wedge e^{1}$. Then taking $i_{V} \circ d$ of this expression and making use of (A.1r), (A.3r), (6.3) and $H=0$ on $\Sigma$, we obtain

$$
\begin{aligned}
i_{V} \circ d\left\{i_{V} \circ d\left(\Theta \wedge e^{1}\right)\right\} & =(g \alpha-f)\left(g T+f e_{2}\right)(H) \Theta \wedge e^{1} \\
& =-(g \alpha-f)^{2} e_{2}(H) \Theta \wedge e^{1}
\end{aligned}
$$

on $\Sigma$. For the last equality we have used $T(H)=-\alpha e_{2}(H)$ since $\alpha e_{2}+T \in T \Sigma$ and $H=0$ on $\Sigma$. Expanding the left-hand side of (A.5r) gives

$$
e_{2}(H)=2 W+e_{1}\left(\omega\left(e_{2}\right)\right)+2 \omega(T)+\left(\omega\left(e_{2}\right)\right)^{2} .
$$

Here we have used (A.6r) and $\omega\left(e_{1}\right)=H=0$ on $\Sigma$. The surfaces $\varphi_{t}\left(\Sigma \backslash S_{\Sigma}\right)$ are the level sets of a defining function $\rho$. Here $\dot{\varphi}_{t}=f e_{2}+g T$. It follows that $\left(f e_{2}+g T\right)(\rho)=1$. On the other hand, $\left(\alpha e_{2}+T\right)(\rho)=0$ from the definition of $\alpha$. So $T(\rho)=-\alpha e_{2}(\rho)$ and $e_{2}(\rho)=(f-\alpha g)^{-1}$ (where $f-\alpha g \neq 0$ ). Applying (A.6r) and (A.7r) to $\rho$, and using the above formulas, we obtain

$$
\begin{aligned}
& \omega\left(e_{2}\right)=h^{-1} e_{1}(h)+2 \alpha, \\
& \omega(T)=e_{1}(\alpha)-\alpha h^{-1} e_{1}(h)-\operatorname{Im} A_{11}
\end{aligned}
$$


where $h=f-\alpha g$. Now substituting (6.6a), (6.6b) into (6.5), we get

$$
e_{2}(H)=2 W-2 \operatorname{Im} A_{11}+4 e_{1}(\alpha)+4 \alpha^{2}+h^{-1} e_{1}^{2}(h)+2 \alpha h^{-1} e_{1}(h) .
$$

Observing that $e_{1}\left(e_{1}\left(h^{2}\right)\right) \Theta \wedge e^{1}=\Theta \wedge d\left(e_{1}\left(h^{2}\right)\right)=-d\left(e_{1}\left(h^{2}\right) \Theta\right)+2 e_{1}\left(h^{2}\right) \alpha e^{1} \wedge \Theta$ on $\Sigma$ by (A.1r) and (6.3), we integrate $\frac{1}{2} e_{1}\left(e_{1}\left(h^{2}\right)\right)=\left(e_{1}(h)\right)^{2}+h e_{1}^{2}(h)$ to obtain

$$
-\int_{\Sigma} h e_{1}^{2}(h) \Theta \wedge e^{1}=\int_{\Sigma}\left[\left(e_{1}(h)\right)^{2}+2 \alpha h e_{1}(h)\right] \Theta \wedge e^{1} .
$$

Substituting (6.7) into (6.4) and (6.4) into (6.1) and using the above formula, we finally reach the following second variation formula.

Proposition 6.1. Suppose the surface $\Sigma$ is p-minimal as defined in Section 2. Let $f, g$ be functions with compact support away from the singular set and the boundary of $\Sigma$. Then

$$
\begin{aligned}
& \delta_{f e_{2}+g T}^{2} \int_{\Sigma} \Theta \wedge e^{1} \\
& =\int_{\Sigma}\left\{\left(e_{1}(f-\alpha g)\right)^{2}+(f-\alpha g)^{2}\left[-2 W+2 \operatorname{Im} A_{11}-4 e_{1}(\alpha)-4 \alpha^{2}\right]\right\} \Theta \wedge e^{1} .
\end{aligned}
$$

Note that the Webster-Tanaka curvature $W$ and the torsion $A_{11}$ are geometric quantities of the ambient pseudohermitian 3-manifold $M$. When the torsion $A_{11}$ vanishes and $W$ is positive, we can easily discuss the stability of a p-minimal surface (see Example 2 in Section 7). If both $W$ and $A_{11}$ vanish, e.g. in the case of $M=H_{1}$ (see the Appendix), we can compute $\alpha, e_{1}(\alpha)$ for a graph $z=u(x, y)$ as follows. First note that the defining function $\rho=(z-u(x, y)) D^{-1}$ satisfies the condition $e_{2}(\rho)=1$ (recall $e_{2}=-\left[\left(u_{x}-y\right) \hat{e}_{1}+\left(u_{y}+x\right) \hat{e}_{2}\right] D^{-1}$ in Section 2). So $\alpha=-T(\rho)=-\partial \rho / \partial z=-D^{-1}$ and a direct computation shows (recall $e_{1}=\left[-\left(u_{y}+x\right) \hat{e}_{1}+\left(u_{x}-y\right) \hat{e}_{2}\right] D^{-1}$ in Section 2) that

$$
\begin{aligned}
-4 e_{1}(\alpha)-4 \alpha^{2}= & 4\left\{\left(u_{x}-y\right)\left(u_{y}+x\right)\left(u_{x x}-u_{y y}\right)\right. \\
& \left.+\left[\left(u_{y}+x\right)^{2}-\left(u_{x}-y\right)^{2}\right] u_{x y}\right\} D^{-4} .
\end{aligned}
$$

For example if $u=x y$, the right-hand side of (6.9) equals $1 / x^{2}$. So away from the singular set, the second variation of the p-area is nonnegative according to (6.8) (it is easy to see that the second variation in the $e_{1}$ direction always vanishes). Note that $\{x=0\}$ is the singular set in this example. From the parea minimizing property shown below (Proposition 6.2), we know the second variation of the $\mathrm{p}$-area for any $\mathrm{p}$-minimal graph over the $x y$-plane with no singular points must be nonnegative.

If we consider only the variation in the $T$ direction, i.e., $f=0$, we should combine the term in (6.9) with terms involving $e_{1}(\alpha)$ in the expansion of $\left(e_{1}(-\alpha g)\right)^{2}$ (to get a better expression of (6.8)). For instance, take a graph 
$(x, y, u(x, y)) \in H_{1}$ over a domain $\Omega$ in the $x y$-plane. We denote the energy functional for the $\mathrm{p}$-area by

$$
E(u)=\int_{\Omega} D d x \wedge d y
$$

in view of (2.5) and (2.11). A direct computation shows that $\left.\frac{d^{2}}{d \varepsilon^{2}}\right|_{\varepsilon=0} E(u+$ $\varepsilon v)=\int_{\Omega} D^{-1}\left(e_{1}(v)\right)^{2} d x \wedge d y$ for a variation $v=v(x, y)$. On the other hand, this should be obtained from (6.8) by letting $f=0$ and $g=v$ (with compact support away from the singular set and $\partial \Omega$ ). It turns out to be equivalent to verifying the following integral formula (note that $\alpha=-D^{-1}, \Theta \wedge e^{1}=$ $D d x \wedge d y)$

$$
\int_{\Omega}\left\{e_{1}\left(v^{2}\right) D^{-1} e_{1}\left(D^{-1}\right)+v^{2}\left[\left(e_{1}\left(D^{-1}\right)\right)^{2}+4 D^{-2} e_{1}\left(D^{-1}\right)-4 D^{-4}\right]\right\} D d x \wedge d y=0 .
$$

We leave this verification to the reader (Hint: we need an integration by parts formula $-\int e_{1}(\varphi) \psi \Theta \wedge e^{1}=-\int\left[\varphi e_{1}(\psi)+2 \varphi \psi \alpha\right] \Theta \wedge e^{1}$. Express $D^{2}=$ $\left(e_{1}(\sigma)\right)^{2}+\left(e_{2}(\sigma)\right)^{2}$ where $\sigma=z-u(x, y)$. The following formulas: $e_{1}^{2}(\sigma)=$ $e_{1}(\sigma)=0, e_{2}(\sigma)=D,\left[e_{1}, e_{2}\right]=-2 \partial_{z}-e_{2}(\theta) e_{2}, e_{1}\left(D^{2}\right)=-4 D-2 e_{2}(\theta) D^{2}$, and $e_{1}^{2}\left(D^{2}\right)=2\left(e_{1}(D)\right)^{2}+4 e_{2}(\theta) D+4\left(e_{2}(\theta) D\right)^{2}$ are useful).

For later use, we deduce a different expression for $\Xi \equiv\left[-2 W+2 \operatorname{Im} A_{11}-\right.$ $\left.4 e_{1}(\alpha)-4 \alpha^{2}\right] \Theta \wedge e^{1}$ in (6.8). Noting that $e^{2} \wedge \Theta=0$ on $\Sigma$, we can easily get

$$
d(\alpha \Theta)=\left[-e_{1}(\alpha)-2 \alpha^{2}\right] \Theta \wedge e^{1}
$$

by (A.1r) and (6.3). From (A.3r), (6.3), and (6.6a), we can relate $\operatorname{Im} A_{11}$ to $\omega(T)$ as follows:

$$
\left(\operatorname{Im} A_{11}\right) \Theta \wedge e^{1}=-d e^{2}-\left[\omega(T)+\alpha h^{-1} e_{1}(h)+2 \alpha^{2}\right] \Theta \wedge e^{1} .
$$

In view of (6.10) and (6.11), we can express $\Xi$ in the following form:

$$
\Xi=-2\left[W+\omega(T)+\alpha h^{-1} e_{1}(h)\right] \Theta \wedge e^{1}+d\left(4 \alpha \Theta-2 e^{2}\right) .
$$

In Euclidean 3-geometry, we take the interior product of the volume form with a vector field normal to a family of surfaces as a calibrating form ([HL]). This 2form restricts to the area form on surfaces, and its exterior differentiation equals the mean curvature times the volume form along a surface. We have analogous results. Suppose $M$ is foliated by a family of surfaces $\Sigma_{t},-\varepsilon<t<\varepsilon$. Let $e_{1}$ be a vector field which is characteristic along any surface $\Sigma_{t}$. We are assuming $\Sigma_{t}$ 's have no singular points. Let $e_{2}=J e_{1}$ denote the Legendrian normal along each $\Sigma_{t}$. Then the 2-form $\Phi=\frac{1}{2} i_{e_{2}}(\Theta \wedge d \Theta)$ satisfies the following properties. First, a direct computation shows that $\Phi=\Theta \wedge e^{1}$, our area 2-form from formula (A.1r). Secondly, $d \Phi=-H \Theta \wedge e^{1} \wedge e^{2}$ by (6.2). So $\left\{\Sigma_{t}\right\}$ are p-minimal surfaces if and only if $d \Phi=0$. Now suppose this is the case and $\Sigma^{\prime}$ 
is a deformed surface with no singular points near a p-minimal surface $\Sigma=\Sigma_{0}$ having the same boundary. Also suppose the Poincare lemma holds. That is to say, there is a 1-form $\Psi$ such that $\Phi=d \Psi$. Then by Stokes' theorem, we have

$$
\text { p-Area }(\Sigma)=\int_{\Sigma} \Phi=\int_{\partial \Sigma} \Psi=\int_{\partial \Sigma^{\prime}} \Psi=\int_{\Sigma^{\prime}} \Phi \text {. }
$$

For $\Sigma^{\prime}$, we have corresponding $e_{1}^{\prime}, e_{2}^{\prime}, e^{1 \prime}, e^{2 \prime}$. There is a function $\alpha^{\prime}$ such that $T+\alpha^{\prime} e_{2}^{\prime}$ is tangent to $\Sigma^{\prime}$. Applying $\Phi=\Theta \wedge e^{1}$ to the basis $\left(T+\alpha^{\prime} e_{2}^{\prime}, e_{1}^{\prime}\right)$ of $T \Sigma^{\prime}$, we obtain $e^{1}\left(e_{1}^{\prime}\right)$. It follows that $\Phi=e^{1}\left(e_{1}^{\prime}\right) \Theta \wedge e^{1 \prime}$ when restricted to $\Sigma^{\prime}$. So we have

$$
\begin{aligned}
\int_{\Sigma^{\prime}} \Phi & =\int_{\Sigma^{\prime}} e^{1}\left(e_{1}^{\prime}\right) \Theta \wedge e^{1 \prime} \\
& \leq \int_{\Sigma^{\prime}} \Theta \wedge e^{1 \prime}=\text { p-Area }\left(\Sigma^{\prime}\right)\left(\text { since } e^{1}\left(e_{1}^{\prime}\right) \leq 1\right) .
\end{aligned}
$$

From (6.13) and (6.14), we have shown that

$$
\operatorname{p}-\operatorname{Area}(\Sigma) \leq \mathrm{p}-\operatorname{Area}\left(\Sigma^{\prime}\right)
$$

Let us summarize the above arguments in the following

Proposition 6.2. Suppose we can foliate an open neighborhood of a p-minimal surface $\Sigma$ by a family of p-minimal surfaces with no singular points, and in this neighborhood the Poincaré lemma holds (i.e., any closed 2-form is exact). Then $\Sigma$ has the local p-area-minimizing property. That is to say, if $\Sigma^{\prime}$ is a deformed surface with no singular points near $\Sigma$ having the same boundary, then (6.15) holds.

We remark that a p-minimal surface in $H_{1}$ with no singular points, which is a graph over the $x y$-plane, satisfies the assumption in Proposition 6.2. Note that a translation of such a p-minimal graph in the $z$-axis is still p-minimal (quantitatively $u+c$ is again a solution if $u=u(x, y)$ is a solution to (pMGE)). Also a vertical (i.e. perpendicular to the $x y$-plane) plane in $H_{1}$ satisfies the assumption in Proposition 6.2. Note that a vertical plane is a p-minimal surface with no singular points, and a family of parallel such surfaces surely foliates an open neighborhood of a given one.

\section{Closed p-minimal surfaces in the 3-sphere and proof of Theorem $\mathbf{E}$}

First let us describe the standard pseudohermitian 3-sphere $\left(S^{3}, \hat{J}, \hat{\Theta}\right)$ (see the Appendix for the definition of basic notions). The unit 3-sphere $S^{3}$ in $C^{2}$ inherits a standard contact structure $\xi=T S^{3} \cap J_{C^{2}}\left(T S^{3}\right)$ where $J_{C^{2}}$ denotes the almost complex structure of $C^{2}$. The standard $C R$ structure $\hat{J}$ compatible with $\xi$ is nothing but the restriction of $J_{C^{2}}$ on $\xi$. Let $r=\left|\zeta^{1}\right|^{2}+\left|\zeta^{2}\right|^{2}-1$ where $\left(\zeta^{1}, \zeta^{2}\right) \in$ $C^{2}$. The contact form $\hat{\Theta} \equiv-i \partial r=-i\left(\bar{\zeta}^{1} d \zeta^{1}+\bar{\zeta}^{2} d \zeta^{2}\right)$ restricted to $S^{3} \equiv\{r=0\}$ 
gives rise to the Reeb vector field $\hat{T}=i \zeta^{1} \partial_{\zeta^{1}}+i \zeta^{2} \partial_{\zeta^{2}}-i \bar{\zeta}^{1} \partial_{\bar{\zeta}^{1}}-i \bar{\zeta}^{2} \partial_{\bar{\zeta}^{2}}$. Take the complex vector field $\hat{Z}_{1}=\bar{\zeta}^{2} \partial_{\zeta^{1}}-\bar{\zeta}^{1} \partial_{\zeta^{2}}$ and the complex 1-form $\hat{\theta}^{1}=$ $\zeta^{2} d \zeta^{1}-\zeta^{1} d \zeta^{2}$ such that $\left\{\hat{\Theta}, \hat{\theta}^{1}, \hat{\theta}^{\overline{1}}\right\}$ is dual to $\left\{\hat{T}, \hat{Z}_{1}, \hat{Z}_{\overline{1}}\right\}$ and $d \hat{\Theta}=i \hat{\theta}^{1} \wedge \hat{\theta}^{\overline{1}}$. It follows that $\hat{\omega}_{1}^{1}=-2 i \hat{\Theta}, \hat{A}_{\overline{1}}^{1}=0$ in the corresponding (A.3) and (A.4) in the Appendix. Also in the corresponding (A.5), $\hat{W}=2$. Write $\hat{Z}_{1}=\frac{1}{2}\left(\hat{e}_{1}-i \hat{e}_{2}\right)$ for real vector fields $\hat{e}_{1}, \hat{e}_{2}$. Let $\hat{\nabla}^{p . h}$. denote the pseudohermitian connection of $(\hat{J}, \hat{\Theta})$. From $\hat{\nabla}^{p \cdot h \cdot} \hat{Z}_{1}=\hat{\omega}_{1}^{1} \otimes \hat{Z}_{1}$ (see (A.2)), we have

$$
\hat{\nabla}^{p \cdot h} \cdot \hat{e}_{1}=-2 \hat{\Theta} \otimes \hat{e}_{2}, \hat{\nabla}^{p \cdot h} \cdot \hat{e}_{2}=2 \hat{\Theta} \otimes \hat{e}_{1} .
$$

Recall that a Legendrian geodesic (with respect to $\hat{\nabla}^{p . h}$ ) is a Legendrian curve $\gamma$ such that $\hat{\nabla}_{\dot{\gamma}}^{p . h .} \dot{\gamma}=0$. Here $\dot{\gamma}=\frac{d \gamma}{d s}$ is the unit tangent vector with respect to the Levi metric and $s$ is a parameter of unit speed. A Legendrian great circle of $\left(S^{3}, \hat{J}, \hat{\Theta}\right)$ is a great circle in the usual sense, whose tangents belong to the kernel of $\hat{\Theta}$.

Lemma 7.1. In $\left(S^{3}, \hat{J}, \hat{\Theta}\right)$ a Legendrian geodesic is a part of a Legendrian great circle, and vice versa.

Proof. Suppose $\gamma$ is a Legendrian geodesic. Write $\dot{\gamma}=a(s) \hat{e}_{1}+b(s) \hat{e}_{2}$ (note that $\hat{e}_{1}$ and $\hat{e}_{2}=\hat{J} \hat{e}_{1}$ belong to, and form a basis of, the kernel of $\hat{\Theta}$ ). Compute $0=\hat{\nabla}_{\dot{\gamma}}^{p . h .} \dot{\gamma}=\dot{a} \hat{e}_{1}+\dot{b} \hat{e}_{2}$ since $\hat{\nabla}_{\dot{\gamma}}^{p . h} \cdot \hat{e}_{1}=\hat{\nabla}_{\dot{\gamma}}^{p . h} \hat{e}_{2}=0$ by $(7.1)$ and $\hat{\Theta}(\dot{\gamma})=$ 0 . So $a$ ( $b$, respectively) is a constant $c_{1}$ (c $c_{2}$, respectively) along $\gamma$. Note that $c_{1}^{2}+c_{2}^{2}=1$ since $a^{2}+b^{2}=1$ by the unity of $\dot{\gamma}$. Now write $\zeta^{1}=x^{1}+i y^{1}$, $\zeta^{2}=x^{2}+i y^{2}$. From the definition we can express

$$
\begin{aligned}
& \hat{e}_{1}=x^{2} \partial_{x^{1}}-y^{2} \partial_{y^{1}}-x^{1} \partial_{x^{2}}+y^{1} \partial_{y^{2}}, \\
& \hat{e}_{2}=y^{2} \partial_{x^{1}}+x^{2} \partial_{y^{1}}-y^{1} \partial_{x^{2}}-x^{1} \partial_{y^{2}} .
\end{aligned}
$$

Writing $\gamma(s)=\left(x^{1}(s), y^{1}(s), x^{2}(s), y^{2}(s)\right)$, we can express the equation $\dot{\gamma}=$ $c_{1} \hat{e}_{1}+c_{2} \hat{e}_{2}$ by (7.2) as

$$
\begin{aligned}
& \dot{x}^{1}=c_{1} x^{2}+c_{2} y^{2}, \dot{y}^{1}=c_{2} x^{2}-c_{1} y^{2}, \\
& \dot{x}^{2}=-c_{1} x^{1}-c_{2} y^{1}, \dot{y}^{2}=-c_{2} x^{1}+c_{1} y^{1} .
\end{aligned}
$$

It is easy to see from (7.3) that $\ddot{x}^{1}=-x^{1}, \ddot{y}^{1}=-y^{1}, \ddot{x}^{2}=-x^{2}, \ddot{y}^{2}=-y^{2}$. Therefore

$$
\left(x^{1}(s), y^{1}(s), x^{2}(s), y^{2}(s)\right)=\cos (s)\left(\alpha_{1}, \alpha_{2}, \alpha_{3}, \alpha_{4}\right)+\sin (s)\left(\beta_{1}, \beta_{2}, \beta_{3}, \beta_{4}\right) .
$$

Here the constant vector $\left(\beta_{1}, \beta_{2}, \beta_{3}, \beta_{4}\right)$ is determined by the constant vector $\left(\alpha_{1}, \alpha_{2}, \alpha_{3}, \alpha_{4}\right)$ as follows:

$$
\left(\beta_{1}, \beta_{2}\right)=\left(\alpha_{3}, \alpha_{4}\right)\left(\begin{array}{cc}
c_{1} & c_{2} \\
c_{2} & -c_{1}
\end{array}\right),\left(\beta_{3}, \beta_{4}\right)=\left(\alpha_{1}, \alpha_{2}\right)\left(\begin{array}{cc}
-c_{1} & -c_{2} \\
-c_{2} & c_{1}
\end{array}\right) .
$$


Using $c_{1}^{2}+c_{2}^{2}=1$, we have $\beta_{1}^{2}+\beta_{2}^{2}=\alpha_{3}^{2}+\alpha_{4}^{2}, \beta_{3}^{2}+\beta_{4}^{2}=\alpha_{1}^{2}+\alpha_{2}^{2}$ by (7.5). Denote $\left(\alpha_{1}, \alpha_{2}, \alpha_{3}, \alpha_{4}\right)$ by $\vec{\alpha}$ and $\left(\beta_{1}, \beta_{2}, \beta_{3}, \beta_{4}\right)$ by $\vec{\beta}$. We can write (7.4) as

$$
\gamma(s)=\cos (s) \vec{\alpha}+\sin (s) \vec{\beta} .
$$

A direct computation using (7.5) and $c_{1}^{2}+c_{2}^{2}=1$ shows that $\vec{\alpha}$ is perpendicular to $\vec{\beta}$ and $|\vec{\alpha}|=|\vec{\beta}|=1$ in $R^{4}$ since $\gamma(s) \in S^{3}$. It is now clear from (7.4') that the Legendrian geodesic $\gamma(s), 0 \leq s \leq 2 \pi$, is a great circle. Moreover, $\vec{\beta}$ sits in the contact plane at the point $\vec{\alpha}$. Write $\hat{\Theta}=x^{1} d y^{1}-y^{1} d x^{1}+x^{2} d y^{2}-y^{2} d x^{2}$. Define $(e, f)^{\perp}=(-f, e)$ for a plane vector $(e, f)$. Write $\vec{\alpha}=\left(\vec{\alpha}_{1}, \vec{\alpha}_{2}\right)$ and $\vec{\beta}$ $=\left(\vec{\beta}_{1}, \vec{\beta}_{2}\right)$ for plane vectors $\vec{\alpha}_{1}, \vec{\alpha}_{2}, \vec{\beta}_{1}, \vec{\beta}_{2}$. Then $\vec{\beta} \in \operatorname{ker} \hat{\Theta}$ at the point $\vec{\alpha}$ if and only if

$$
\left(\vec{\alpha}_{1}^{\perp}, \vec{\alpha}_{2}^{\perp}\right) \cdot\left(\vec{\beta}_{1}, \vec{\beta}_{2}\right)=\vec{\alpha}_{1}^{\perp} \cdot \vec{\beta}_{1}+\vec{\alpha}_{2}^{\perp} \cdot \vec{\beta}_{2}=0
$$

(in which "." denotes the inner product). Now it is easy to see that (7.5) implies (7.6) for $\vec{\alpha}_{1}=\left(\alpha_{1}, \alpha_{2}\right), \vec{\alpha}_{2}=\left(\alpha_{3}, \alpha_{4}\right), \vec{\beta}_{1}=\left(\beta_{1}, \beta_{2}\right), \vec{\beta}_{2}=\left(\beta_{3}, \beta_{4}\right)$. Conversely, given an arbitrary point $\vec{\alpha} \in S^{3}$ and a unit tangent vector $\vec{\beta}$ in the contact plane at $\vec{\alpha}$, we claim that the great circle $\gamma(s)$ defined by $\left(7.4^{\prime}\right)$ is Legendrian and a Legendrian geodesic. From $\dot{\gamma}(s)=-\sin (s) \vec{\alpha}+\cos (s) \vec{\beta}$, we compute

$$
\begin{aligned}
& \left\{\cos (s)\left(\vec{\alpha}_{1}^{\perp}, \vec{\alpha}_{2}^{\perp}\right)+\sin (s)\left(\vec{\beta}_{1}^{\perp}, \vec{\beta}_{2}^{\perp}\right)\right\} \cdot\left\{-\sin (s)\left(\vec{\alpha}_{1}, \vec{\alpha}_{2}\right)+\cos (s)\left(\vec{\beta}_{1}, \vec{\beta}_{2}\right)\right\} \\
& =-\sin ^{2}(s)\left(\vec{\beta}_{1}^{\perp} \cdot \vec{\alpha}_{1}+\vec{\beta}_{2}^{\perp} \cdot \vec{\alpha}_{2}\right)+\cos ^{2}(s)\left(\vec{\alpha}_{1}^{\perp} \cdot \vec{\beta}_{1}+\vec{\alpha}_{2}^{\perp} \cdot \vec{\beta}_{2}\right) \\
& =\vec{\alpha}_{1}^{\perp} \cdot \vec{\beta}_{1}+\vec{\alpha}_{2}^{\perp} \cdot \vec{\beta}_{2} \quad\left(\text { since } \vec{\eta} \cdot \vec{\zeta}=\vec{\eta}^{\perp} \cdot \vec{\zeta}^{\perp} \quad \text { and }\left(\vec{\eta}^{\perp}\right)^{\perp}=-\vec{\eta}\right) \\
& =0 \quad(\text { by (7.6)). }
\end{aligned}
$$

So $\gamma(s)$ is Legendrian by (7.6) again. From (7.2) we can express $\hat{e}_{1}, \hat{e}_{2}$ at $\gamma(s)$ as follows:

$$
\begin{aligned}
& \hat{e}_{1}(\gamma(s))=\cos (s)\left(\alpha_{3},-\alpha_{4},-\alpha_{1}, \alpha_{2}\right)+\sin (s)\left(\beta_{3},-\beta_{4},-\beta_{1}, \beta_{2}\right), \\
& \hat{e}_{2}(\gamma(s))=\cos (s)\left(\alpha_{4}, \alpha_{3},-\alpha_{2},-\alpha_{1}\right)+\sin (s)\left(\beta_{4}, \beta_{3},-\beta_{2},-\beta_{1}\right) .
\end{aligned}
$$

Recall that we write $\vec{\alpha}=\left(\alpha_{1}, \alpha_{2}, \alpha_{3}, \alpha_{4}\right), \vec{\beta}=\left(\beta_{1}, \beta_{2}, \beta_{3}, \beta_{4}\right)$. Equating $(\dot{\gamma}(s)$ $=)-\sin (s) \vec{\alpha}+\cos (s) \vec{\beta}=c_{1} \hat{e}_{1}(\gamma(s))+c_{2} \hat{e}_{2}(\gamma(s))$ with $c_{1}^{2}+c_{2}^{2}=1$ gives the equations (7.5) (requiring $c_{1}^{2}+c_{2}^{2}=1$ gets rid of other equivalent equations). Solving (7.5) for $c_{1}, c_{2}$, we obtain $c_{1}=\left(\beta_{1} \alpha_{3}-\beta_{2} \alpha_{4}\right)\left(\alpha_{3}^{2}+\alpha_{4}^{2}\right)^{-1}, c_{2}=\left(\beta_{1} \alpha_{4}+\right.$ $\left.\beta_{2} \alpha_{3}\right)\left(\alpha_{3}^{2}+\alpha_{4}^{2}\right)^{-1}$ if $\alpha_{3}^{2}+\alpha_{4}^{2} \neq 0$ or $c_{1}=\left(-\alpha_{1} \beta_{3}+\alpha_{2} \beta_{4}\right)\left(\alpha_{1}^{2}+\alpha_{2}^{2}\right)^{-1}, c_{2}=$ $\left(-\alpha_{1} \beta_{4}-\alpha_{2} \beta_{3}\right)\left(\alpha_{1}^{2}+\alpha_{2}^{2}\right)^{-1}$ if $\alpha_{1}^{2}+\alpha_{2}^{2} \neq 0$. Note that two expressions for $c_{1}\left(c_{2}\right.$, respectively) are equal where $\alpha_{1}^{2}+\alpha_{2}^{2} \neq 0$ and $\alpha_{3}^{2}+\alpha_{4}^{2} \neq 0$ by the condition (7.6) and $\vec{\alpha} \cdot \vec{\beta}=0$. Now $\hat{\nabla}_{\dot{\gamma}}^{\text {p.h. }} \dot{\gamma}=c_{1} \hat{\nabla}_{\dot{\gamma}}^{\text {p.h. }} \hat{e}_{1}(\gamma(s))+c_{2} \hat{\nabla}_{\dot{\gamma}}^{\text {p.h. }} \hat{e}_{2}(\gamma(s))$ $=0$ by (7.1). We have proved that $\gamma(s)$ is a Legendrian geodesic.

Recall (see Section 2) that the p-mean curvature $H$ of a surface $\Sigma$ in a pseudohermitian 3-manifold $(M, J, \Theta)$ depends on $(J, \Theta)$. Let $\tilde{H}$ denote the p-mean curvature associated to another contact form $\tilde{\Theta}=\lambda^{2} \Theta, \lambda>0$ with $J$ fixed. Let $e_{2}$ denote the Legendrian normal to $\Sigma$. Then we have the following transformation law. 
Lemma 7.2. Suppose $\tilde{\Theta}=\lambda^{2} \Theta, \lambda>0$. Then $\tilde{H}=\lambda^{-2}\left(\lambda H-3 e_{2}(\lambda)\right)$.

Proof. Let $\tilde{e}_{1}$ denote the characteristic field with respect to $\tilde{\Theta}$. Then it follows from the definition that $\tilde{e}_{1}=\lambda^{-1} e_{1}$. Applying (5.7) in [Lee] to $e_{1}$ (in our case, $\left.n=1, Z_{1}=\frac{1}{2}\left(e_{1}-i e_{2}\right)\right)$, we obtain

$$
\lambda \tilde{\omega}_{1}^{1}\left(\tilde{e}_{1}\right)=\omega_{1}^{1}\left(e_{1}\right)-3 i \lambda^{-1} e_{2}(\lambda) .
$$

Note that $H=\omega\left(e_{1}\right)=-i \omega_{1}{ }^{1}\left(e_{1}\right)$ (see the remark after (2.8)). Rewriting (7.7) in terms of $H$ and $\tilde{H}$ gives what we want.

We define the Cayley transform $F: S^{3} \backslash\{(0,-1)\} \rightarrow H_{1}$ by

$$
x=\operatorname{Re}\left(\frac{\zeta^{1}}{1+\zeta^{2}}\right), y=\operatorname{Im}\left(\frac{\zeta^{1}}{1+\zeta^{2}}\right), z=\frac{1}{2} \operatorname{Re}\left[i\left(\frac{1-\zeta^{2}}{1+\zeta^{2}}\right)\right]
$$

where $\left(\zeta^{1}, \zeta^{2}\right) \in S^{3} \subset C^{2}$ satisfies $\left|\zeta^{1}\right|^{2}+\left|\zeta^{2}\right|^{2}=1$ (see, e.g., [JL1]). A direct computation shows that

$$
\hat{\Theta}=F^{*}\left(\lambda^{2} \Theta_{0}\right)
$$

where $\lambda^{2}=4\left[4 z^{2}+\left(x^{2}+y^{2}+1\right)^{2}\right]^{-1}$ (recall that $\Theta_{0}=d z+x d y-y d x$ is the standard contact form for $H_{1}$ ).

Lemma 7.3. Let $\Sigma$ be a $C^{2}$ smoothly embedded p-minimal surface in $\left(S^{3}, \hat{J}, \hat{\Theta}\right)$. Suppose $p \in \Sigma$ is an isolated singular point. Then there exists a neighborhood $V$ of $p$ in $\Sigma$ such that $V$ is contained in the union of all Legendrian great circles through $p$.

Proof. Without loss of generality, we may assume $(0,-1) \notin \Sigma$. Consider $\Sigma_{0}$ $=F(\Sigma) \subset H_{1}$. Let $H_{0}$ denote the p-mean curvature of $\Sigma_{0}$ in $H_{1}$. By (7.8) and Lemma 7.2, we obtain $H_{0}=3 \lambda^{-1} e_{2}(\lambda)$. Here $e_{2}$ is the Legendrian normal of $\Sigma_{0}$. Recall (Section 2) that $e_{2}=-(\cos \theta) \hat{e}_{1}-(\sin \theta) \hat{e}_{2}$. So $e_{2}(\lambda)=$ $-(\cos \theta) \hat{e}_{1}(\lambda)-(\sin \theta) \hat{e}_{2}(\lambda)$ is bounded near the isolated singular point $F(p)$ since $\hat{e}_{1}(\lambda)$ and $\hat{e}_{2}(\lambda)$ are global $C^{\infty}$ smooth functions. Thus $H_{0}$ is bounded near $F(p)$. (Note that near a singular point, $\Sigma_{0}$ is a graph over the $x y$-plane.) Observing that characteristic curves and the singular set are preserved under the contact diffeomorphism $F$, we conclude the proof of the lemma by Theorem 3.10 and Lemma 7.1.

A direct proof of Corollary $F$ (for the nonexistence of hyperbolic p-minimal surfaces). Let $\Sigma$ be a closed, connected, $C^{2}$ smoothly embedded p-minimal surface in $\left(S^{3}, \hat{J}, \hat{\Theta}\right)$. Without loss of generality, we may assume $(0,-1) \notin \Sigma$. Consider $\Sigma_{0}=F(\Sigma) \subset H_{1}$. As argued in the proof of Lemma 7.3, $H_{0}$, the p-mean curvature of $\Sigma_{0}$, is bounded. According to Theorem B any singular point of $\Sigma_{0}$ is either isolated or contained in a $C^{1}$ smooth singular curve with no other singular points near it (note that near a singular point, $\Sigma_{0}$ is a graph over the $x y$-plane). So back to $\Sigma$ through $F^{-1}$, we can only have isolated singular points or closed singular curves on $\Sigma$. Similarly via the Cayley transform we 
can have an extension theorem analogous to Corollary 3.6 with the characteristic curve replaced by a characteristic (Legendrian) great circle (arc) in the case of $\left(S^{3}, \hat{J}, \hat{\Theta}\right)$.

Now suppose $\Sigma$ has an isolated singular point $p$. By Lemma 7.3 there exists a neighborhood $V$ of $p$ in $\Sigma$ such that $V$ is contained in the union of all Legendrian great circles through $p$. But this union simply forms a pminimal 2-sphere. This 2 -sphere must be the whole $\Sigma$ since $\Sigma$ is connected. Next suppose $\Sigma$ does not have any isolated singular point. Then in view of the extension theorem, the space of leaves (Legendrian great circles) of the characteristic foliation (including touching points on singular curves) forms a closed, connected, 1-dimensional manifold. So it must be homeomorphic to $S^{1}$. In this case, $\Sigma$ is topologically a torus.

Example 1. Every coordinate sphere (defined by $x^{1}, y^{1}, x^{2}$ or $y^{2}=0$ ) is a closed, connected, embedded p-minimal surface of genus 0 in $\left(S^{3}, \hat{J}, \hat{\Theta}\right)$. For instance, we can write $\left\{y^{2}=0\right\}$ as the union of Legendrian great circles: $\gamma_{t}(s)$ $=\cos (s)(0,0,1,0)+\sin (s)(\cos (t), \sin (t), 0,0)$ parametrized by $t$ (it is a simple exercise to verify (7.6)).

Example 2. Write $\zeta^{1}=\rho_{1} e^{i \varphi_{1}}, \zeta^{2}=\rho_{2} e^{i \varphi_{2}}$ in polar coordinates with $\rho_{1}^{2}+\rho_{2}^{2}=$ 1 on $S^{3}$. We consider the surface $\Sigma_{c}$ defined by $\rho_{1}=c$, a constant between 0 and 1. Note that $\hat{\Theta}=\rho_{1}^{2} d \varphi_{1}+\rho_{2}^{2} d \varphi_{2}, \hat{J} \partial_{\varphi_{j}}=-\rho_{j} \partial_{\rho_{j}}$ and $\hat{J} \partial_{\rho_{j}}=\rho_{j}^{-1} \partial_{\varphi_{j}}$, $j=1,2$ in polar coordinates. Here we have used $\partial_{\varphi_{j}}=i \zeta^{j} \partial_{\zeta^{j}}-i \bar{\zeta}^{j} \partial_{\bar{\zeta}^{j}}, \partial_{\rho_{j}}$ $=\rho_{j}^{-1}\left(\zeta^{j} \partial_{\zeta^{j}}+\bar{\zeta}^{j} \partial_{\bar{\zeta}^{j}}\right.$ ) for $j=1,2$ (no summation convention here). Next we compute the Reeb vector field $T$, the characteristic field $e_{1}$, and the Legendrian normal $e_{2}$ as follows:

$$
T=\partial_{\varphi_{1}}+\partial_{\varphi_{2}}, e_{1}=\frac{\rho_{2}}{\rho_{1}} \partial_{\varphi_{1}}-\frac{\rho_{1}}{\rho_{2}} \partial_{\varphi_{2}}, e_{2}=\hat{J} e_{1}=-\rho_{2} \partial_{\rho_{1}}+\rho_{1} \partial_{\rho_{2}} .
$$

We then have $e^{1}=\rho_{1} \rho_{2}\left(d \varphi_{1}-d \varphi_{2}\right)$ and $e^{2}=-\rho_{2} d \rho_{1}+\rho_{1} d \rho_{2}$. So we can compute the p-area 2-form $\hat{\Theta} \wedge e^{1}=-\rho_{1} \rho_{2} d \varphi_{1} \wedge d \varphi_{2}$, the volume form $\hat{\Theta} \wedge e^{1} \wedge e^{2}$ $=\rho_{1} d \rho_{1} \wedge d \varphi_{1} \wedge d \varphi_{2}$ and $d\left(\hat{\Theta} \wedge e^{1}\right)=-\left(\rho_{2}^{2}-\rho_{1}^{2}\right) \rho_{2}^{-1} d \rho_{1} \wedge d \varphi_{1} \wedge d \varphi_{2}$ (noting that $\left.\rho_{1} d \rho_{1}+\rho_{2} d \rho_{2}=0\right)$. By (6.2) we obtain the p-mean curvature $H=$ $\left(\rho_{2}^{2}-\rho_{1}^{2}\right)\left(\rho_{1} \rho_{2}\right)^{-1}=\rho_{2} \rho_{1}^{-1}-\rho_{1} \rho_{2}^{-1}$ for $\Sigma_{c}$ where $0<\rho_{1}=c<1$ and $\rho_{2}=\sqrt{1-c^{2}}$. Thus for $c=\sqrt{2} / 2\left(\rho_{1}=\rho_{2}=c\right), \Sigma_{c}$ is a closed, connected, embedded p-minimal torus with no singular points (observing that $T$ is tangent at every point of $\Sigma_{c}$ ) and the union of Legendrian great circles defined by $\varphi_{1}+\varphi_{2}=a, 0 \leq a<2 \pi$ with 0 identified with $2 \pi$. In any case, $\Sigma_{c}$ is a torus of constant p-mean curvature. Also note that the p-minimal torus $\Sigma_{\sqrt{2} / 2}$ is not stable. This can be seen from the second variation formula (6.8) in which $\alpha=0, A_{11}=0$, and $W=2$.

To generalize Corollary F, we need to extend the theory of singular set in Section 3 to the general situation. Let $\Sigma$ be a surface in an arbitrary 3dimensional pseudohermitian manifold. Let $p_{0}$ be a singular point of $\Sigma$. Near 
$p_{0}$ let $e_{1}, e_{2} \in \xi$ denote an orthonormal basis with respect to the Levi metric $G$ such that $\frac{1}{2}\left(e_{1}-i e_{2}\right)$ forms a special frame in the sense of [JL2]. Then in pseudohermitian normal coordinates $x, y, z$ associated to this special frame, we can describe $\Sigma$ by a graph $z=u(x, y)$ with $p_{0}=(0,0,0)$. Furthermore, we can write

$$
\begin{aligned}
& e_{1}=\hat{e}_{1}+\mathcal{O}_{1} \\
& e_{2}=\hat{e}_{2}+\mathcal{O}_{2}
\end{aligned}
$$

where $\hat{e}_{1} \equiv \partial_{x}+y \partial_{z}, \hat{e}_{2} \equiv \partial_{y}-x \partial_{z}$, and $\mathcal{O}_{1}, \mathcal{O}_{2}$ are real vector fields of degree $\geq 1$ ([JL2]). Let $\psi=u(x, y)-z$ be a defining function for $\Sigma$. Recall that $\nabla_{b} \psi$ $=\left(e_{1} \psi\right) e_{1}+\left(e_{2} \psi\right) e_{2}$ and $\mathcal{D} \equiv\left|\nabla_{b} \psi\right|_{G} \equiv \sqrt{\left(e_{1} \psi\right)^{2}+\left(e_{2} \psi\right)^{2}}$ (see Section 2). We compute the p-mean curvature (see (pMCE). Note that we take the positive sign below to be consistent with the Heisenberg group case in which we use $z-u(x, y)$ as a defining function):

$$
\begin{aligned}
H & =\operatorname{div}_{b}\left(\frac{\nabla_{b} \psi}{\left|\nabla_{b} \psi\right|_{G}}\right) \\
& =G\left(\nabla_{e_{1}}^{p . h .} \frac{\nabla_{b} \psi}{\left|\nabla_{b} \psi\right|_{G}}, e_{1}\right)+G\left(\nabla_{e_{2}}^{p . h .} \frac{\nabla_{b} \psi}{\left|\nabla_{b} \psi\right|_{G}}, e_{2}\right) \\
& =\left[e_{1}\left(\frac{e_{1} \psi}{\mathcal{D}}\right)+e_{2}\left(\frac{e_{2} \psi}{\mathcal{D}}\right)\right]-\frac{e_{2} \psi}{\mathcal{D}} B_{1}-\frac{e_{1} \psi}{\mathcal{D}} B_{2}
\end{aligned}
$$

where $B_{1}$ and $B_{2}$ are determined by $\nabla_{e_{1}}^{p . h \cdot} e_{1}=B_{1} e_{2}$ and $\nabla_{e_{2}}^{p . h} e_{2}=B_{2} e_{1}$, respectively. Note that both $\frac{e_{1} \psi}{\mathcal{D}}$ and $\frac{e_{2} \psi}{\mathcal{D}}$ are bounded by 1 . Also since $e_{1}, e_{2}$ are local $C^{\infty}$ smooth vector fields, $B_{1}$ and $B_{2}$ are bounded in a neighborhood of $p_{0}$. It follows from (7.10) that near $p_{0}$ where $\mathcal{D}=0$ (i.e., $e_{1} \psi=e_{2} \psi=0$ )

$$
H=\left[e_{1}\left(\frac{e_{1} \psi}{\mathcal{D}}\right)+e_{2}\left(\frac{e_{2} \psi}{\mathcal{D}}\right)\right]+\text { a bounded function. }
$$

To generalize the results in Section 3, we replace $u_{x}-y$ and $u_{y}+x$ by $e_{1} \psi$ and $e_{2} \psi$, respectively in all the places. Thus the singular set $S(u)$ consists of the points where $e_{1} \psi=e_{2} \psi=0$ while $F_{a, b}$ and $U$ are defined to be $a\left(e_{1} \psi\right)$ $+b\left(e_{2} \psi\right)$ and

$$
\left[\begin{array}{ll}
\left(e_{1} \psi\right)_{x} & \left(e_{1} \psi\right)_{y} \\
\left(e_{2} \psi\right)_{x} & \left(e_{2} \psi\right)_{y}
\end{array}\right]
$$

respectively. Note that the above $U$ is reduced to $(U)$ in the beginning of Section 3 at $p_{0}$ by (7.9). It is now easy to see that Lemma 3.1 and Lemma 3.2 continue to hold in the general situation.

To generalize Theorem 3.3, we need to modify the argument used to show that (2) $\Rightarrow$ (3). Let $N(u) \equiv \mathcal{D}^{-1}\left(e_{1} \psi, e_{2} \psi\right)$. Let $\eta_{1}=\mathcal{O}_{1}(x) d y-\mathcal{O}_{1}(y) d x$, $\eta_{2}=\mathcal{O}_{2}(x) d y-\mathcal{O}_{2}(y) d x$ (note that $\left.e_{1}\right\lrcorner d x \wedge d y=d y+\eta_{1}$ and $\left.e_{2}\right\lrcorner d x \wedge d y$ $\left.=-d x+\eta_{2}\right)$. Making use of the formula: $\left.\iint v(f) d x \wedge d y=\oint f(v\lrcorner d x \wedge d y\right)$ $\left.-\iint f d(v\lrcorner d x \wedge d y\right)$ for a vector field $v$ and a function $f$, we compute

$$
\iint_{\Omega_{i}}\left[e_{1}\left(\frac{e_{1} \psi}{\mathcal{D}}\right)+e_{2}\left(\frac{e_{2} \psi}{\mathcal{D}}\right)\right] d x \wedge d y=\oint_{\partial \Omega_{i}} N(u) \cdot v d s+E_{1}+E_{2}
$$


where the unit outward normal $v=\left(\frac{d y}{d s},-\frac{d x}{d s}\right)$ and two error terms $E_{1}, E_{2}$ are given by

$$
\begin{aligned}
& E_{1}=-\iint_{\Omega_{i}}\left(\frac{e_{1} \psi}{\mathcal{D}} d \eta_{1}+\frac{e_{2} \psi}{\mathcal{D}} d \eta_{2}\right) \\
& E_{2}=\oint_{\partial \Omega_{i}} \frac{e_{1} \psi}{\mathcal{D}} \eta_{1}+\frac{e_{2} \psi}{\mathcal{D}} \eta_{2} .
\end{aligned}
$$

Observe that $\frac{e_{1} \psi}{\mathcal{D}}$ and $\frac{e_{2} \psi}{\mathcal{D}}$ are bounded by $1, \eta_{j}=o(d r)$ (little "o"), and $d \eta_{j}$ $=o(d x \wedge d y)$ (little "o"). By (7.12) we can then estimate

$$
\begin{aligned}
& \mid \iint_{\Omega_{i}}[H-\text { (a bounded function) }] d x \wedge d y \mid \\
& =\left|\oint_{\partial \Omega_{i}} N(u) \cdot v d s+E_{1}+E_{2}\right| \\
& \geq \frac{\left|c_{1}-c_{2}\right|}{2}\left|r\left(\tilde{e}_{i}\right)-r\left(e_{i}\right)\right|
\end{aligned}
$$

for large $i$. On the other hand, (3.6) still holds for $H$ replaced by $H$-(a bounded function). So we get a contradiction, and hence validate Theorem 3.3 in the general situation. To generalize other results except Lemma 3.4 in Section 3, we observe that the error terms do not affect the major estimates in the proofs (Lemma 3.4 is used to let us assume that the $x$-axis through $p_{0}$ is transverse to the singular curve in the proof of Proposition 3.5. We may instead assume the $y$-axis through $p_{0}$ is transverse to the singular curve if the $x$-axis is not without using Lemma 3.4. Note that Lemma 3.4 is mainly used in the proof of Theorem A in Section 4). Therefore they all continue to hold in the general situation.

Proof of Theorem E. Locally near a singular point $p_{0}$ of $\Sigma$, we may assume that $\Sigma$ is a $C^{2}$ smooth graph over the $x y$-plane in pseudohermitian normal coordinates $x, y, z$ with $p_{0}=(0,0,0)$. So the singular set $S_{\Sigma}$ (depending only on $\Sigma$ and the contact structure) consists of finitely many isolated points and $C^{1}$ smooth closed curves in view of generalized Theorem B (see the paragraph above). Also the (generalized) extension theorems (Corollary 3.6 and Theorem 3.10) hold in this situation. Now the configuration of characteristic foliation on $\Sigma$ is clear. The associated line field (extended to include those defined on points of singular curves) has only isolated singular points of index 1 in view of (generalized) Lemma 3.8. Therefore the total index sum of this line field is nonnegative. This index sum is equal to the Euler characteristic number of the surface $\Sigma$ according to the Hopf index theorem for a line field (e.g., $[\mathrm{Sp}])$. On the other hand, the Euler characteristic number of $\Sigma$ equals $2-2 g(\Sigma)$ where $g(\Sigma)$ denotes the genus of $\Sigma$. It follows that $g(\Sigma) \leq 1$. 


\section{Appendix. Basic facts in pseudohermitian geometry}

Let $M$ be a smooth (paracompact) 3-manifold. A contact structure or bundle $\xi$ on $M$ is a completely nonintegrable plane distribution. A contact form is a 1-form annihilating $\xi$. Let $(M, \xi)$ be a contact 3-manifold with an oriented contact structure $\xi$. We say a contact form $\Theta$ is oriented if $d \Theta(u, v)>0$ for $(u, v)$ being an oriented basis of $\xi$. There always exists a global oriented contact form $\Theta$, obtained by patching together local ones with a partition of unity. The Reeb vector field of $\Theta$ is the unique vector field $T$ such that $\Theta(T)=1$ and $\mathcal{L}_{T} \Theta=0$ or $d \Theta(T, \cdot)=0$. A $C R$-structure compatible with $\xi$ is a smooth endomorphism $J: \xi \rightarrow \xi$ such that $J^{2}=-$ Identity. We say $J$ is oriented if $(X, J X)$ is an oriented basis of $\xi$ for any nonzero $X \in \xi$. A pseudohermitian structure compatible with $\xi$ is a $C R$-structure $J$ compatible with $\xi$ together with a global contact form $\Theta$.

Given a pseudohermitian structure $(J, \Theta)$, we can choose a complex vector field $Z_{1}$, an eigenvector of $J$ with eigenvalue $i$, and a complex 1 -form $\theta^{1}$ such that $\left\{\Theta, \theta^{1}, \theta^{\overline{1}}\right\}$ is dual to $\left\{T, Z_{1}, Z_{\overline{1}}\right\}\left(\theta^{\overline{1}}=\left(\bar{\theta}^{\overline{1}}\right), Z_{\overline{1}}=\left(\bar{Z}_{1}\right)\right)$. It follows that $d \Theta=i h_{1 \overline{1}} \theta^{1} \wedge \theta^{\overline{1}}$ for some nonzero real function $h_{1 \overline{1}}$. If both $J$ and $\Theta$ are oriented, then $h_{11}$ is positive. In this case we call such a pseudohermitian structure $(J, \Theta)$ oriented, and we can choose a $Z_{1}$ (hence $\theta^{1}$ ) such that $h_{1 \overline{1}}=1$. That is to say

$$
d \Theta=i \theta^{1} \wedge \theta^{\overline{1}} .
$$

We will always assume our pseudohermitian structure is oriented and $h_{1 \overline{1}}=1$. The pseudohermitian connection of $(J, \Theta)$ is the connection $\nabla^{p . h .}$ on $T M \otimes C$ (and extended to tensors) given by

$$
\nabla^{p \cdot h \cdot} Z_{1}=\omega_{1}^{1} \otimes Z_{1}, \nabla^{p \cdot h \cdot} Z_{\overline{1}}=\omega_{\overline{1}}^{\overline{1}} \otimes Z_{\overline{1}}, \nabla^{p \cdot h \cdot} T=0
$$

in which the 1 -form $\omega_{1}{ }^{1}$ is uniquely determined by the following equation with a normalization condition ([Ta, We $]$ ):

$$
\begin{gathered}
d \theta^{1}=\theta^{1} \wedge \omega_{1}{ }^{1}+A^{1}{ }_{\overline{1}} \Theta \wedge \theta^{\overline{1}}, \\
\omega_{1}{ }^{1}+\omega_{\overline{1}}{ }^{\overline{1}}=0 .
\end{gathered}
$$

The coefficient $A^{1}{ }_{\overline{1}}$ in (A.3) is called the (pseudohermitian) torsion. Since $h_{1 \overline{1}}=1, A_{\overline{1} \overline{1}}=h_{1 \overline{1}} A^{1}{ }_{\overline{1}}=A^{1}{ }_{\overline{1}}$. And $A_{11}$ is just the complex conjugate of $A_{\overline{1} \overline{1}}$. Differentiating $\omega_{1}^{1}$ gives

$$
d \omega_{1}{ }^{1}=W \theta^{1} \wedge \theta^{\overline{1}}+2 i \operatorname{Im}\left(A_{11, \overline{1}} \theta^{1} \wedge \Theta\right)
$$

where $W$ is the Tanaka-Webster curvature. Write $\omega_{1}{ }^{1}=i \omega$ for some real 1form $\omega$ by (A.4). This $\omega$ is just the one used in previous sections. Write $Z_{1}=\frac{1}{2}\left(e_{1}-i e_{2}\right)$ for real vectors $e_{1}, e_{2}$. It follows that $e_{2}=J e_{1}$. Let $e^{1}=$ $\operatorname{Re}\left(\theta^{1}\right), e^{2}=\operatorname{Im}\left(\theta^{1}\right)$. Then $\left\{e^{0}=\Theta, e^{1}, e^{2}\right\}$ is dual to $\left\{e_{0}=T, e_{1}, e_{2}\right\}$. Now in 
view of (A.1), (A.2) and (A.3), we have the following real version of structure equations:

$$
\begin{aligned}
d \Theta & =2 e^{1} \wedge e^{2}, \\
\nabla^{p h h} e_{1} & =\omega \otimes e_{2}, \nabla^{p . h} \cdot e_{2}=-\omega \otimes e_{1}, \\
d e^{1} & =-e^{2} \wedge \omega \bmod \Theta ; \quad d e^{2}=e^{1} \wedge \omega \bmod \Theta .
\end{aligned}
$$

Similarly, from (A.5), we have the following equation for $W$ :

$$
d \omega\left(e_{1}, e_{2}\right)=-2 W .
$$

Also by (A.1), (A.3) we can deduce

$$
\begin{aligned}
{\left[Z_{\overline{1}}, Z_{1}\right] } & =i T+\omega_{1}{ }^{1}\left(Z_{\overline{1}}\right) Z_{1}-\omega_{\overline{1}}{ }^{\overline{1}}\left(Z_{1}\right) Z_{\overline{1}}, \\
{\left[Z_{\overline{1}}, T\right] } & =A^{1}{ }_{1} Z_{1}-\omega_{\overline{1}}{ }^{\overline{1}}(T) Z_{\overline{1}} .
\end{aligned}
$$

The real version of (A.6), (A.7) reads

$$
\begin{aligned}
& {\left[e_{1}, e_{2}\right]=-2 T-\omega\left(e_{1}\right) e_{1}-\omega\left(e_{2}\right) e_{2},} \\
& {\left[e_{1}, T\right]=\left(\operatorname{Re} A_{11}\right) e_{1}-\left(\left(\operatorname{Im} A_{11}\right)+\omega(T)\right) e_{2},} \\
& {\left[e_{2}, T\right]=-\left(\left(\operatorname{Im} A_{\overline{1} \overline{1}}\right)+\omega(T)\right) e_{1}+\left(\operatorname{Re} A_{\overline{1} \overline{1}}\right) e_{2} .}
\end{aligned}
$$

Note that $A_{\overline{1} \overline{1}}=A_{1}^{1}$ since $h_{1 \overline{1}}=1$. We define the subgradient operator $\nabla_{b}$ acting on a smooth function $f$ by

$$
\nabla_{b} f=2\left\{\left(Z_{\overline{1}} f\right) Z_{1}+\left(Z_{1} f\right) Z_{\overline{1}}\right\} .
$$

It is easy to see that the definition of $\nabla_{b}$ is independent of the choice of unitary $\left(h_{1 \overline{1}}=1\right)$ frame $Z_{1}$. The real version of (A.8) reads

$$
\nabla_{b} f=\left(e_{1} f\right) e_{1}+\left(e_{2} f\right) e_{2} .
$$

Next we will introduce the 3-dimensional Heisenberg group $H_{1}$ (see, e.g., [FS]). For two points $(x, y, z),\left(x^{\prime}, y^{\prime}, z^{\prime}\right) \in \mathbb{R}^{3}$, we define the multiplication as follows: $(x, y, z) \circ\left(x^{\prime}, y^{\prime}, z^{\prime}\right)=\left(x+x^{\prime}, y+y^{\prime}, z+z^{\prime}+y x^{\prime}-x y^{\prime}\right) . \mathbb{R}^{3}$ endowed with this multiplication "o" forms a Lie group, called the (3-dimensional) Heisenberg group and denoted as $H_{1}$. It is a simple exercise to verify that:

$$
\hat{e}_{1}=\frac{\partial}{\partial x}+y \frac{\partial}{\partial z}, \hat{e}_{2}=\frac{\partial}{\partial y}-x \frac{\partial}{\partial z}, T_{0}=\frac{\partial}{\partial z}
$$

form a basis for the left-invariant vector fields on $H_{1}$. We can endow $H_{1}$ with a standard pseudohermitian structure. The plane distribution spanned by $\hat{e}_{1}, \hat{e}_{2}$ forms a contact structure $\xi_{0}$ (so that $\hat{e}_{1}, \hat{e}_{2}$ are Legendrian, i.e., sitting in the 
contact plane). The $C R$ structure $J_{0}$ compatible with $\xi_{0}$ is defined by $J_{0}\left(\hat{e}_{1}\right)$ $=\hat{e}_{2}, J_{0}\left(\hat{e}_{2}\right)=-\hat{e}_{1}$. The contact form $\Theta_{0}=d z+x d y-y d x$ gives rise to the Reeb vector field $T_{0}=\frac{\partial}{\partial z}$. Observe that $\left\{d x, d y, \Theta_{0}\right\}$ is dual to $\left\{\hat{e}_{1}, \hat{e}_{2}, T_{0}\right\}$ and $d(d x+i d y)=0$. It follows from the structural equations (A.3), (A.4) and (A.5) that the connection form associated to the coframe $d x+i d y$, the torsion and the Tanaka-Webster curvature are all zero.

\section{References}

[B] Z. BALOGH, Size of characteristic sets and functions with prescribed gradient, J. Reine Angew. Math. 564 (2003), 63-83.

[CDG] L. Capogna, D. Danielli and N. Garofalo, The geometric Sobolev embedding fir vector fields and the isoperimetric inequality, Comm. Anal. Geom. 2 (1994), 203-215.

[CF] P. Concus and R. FInN, On capillary free surfaces in the absence of gravity, Acta Math. 132 (1974), 177-198.

[CH] J.-H. ChEng and J.-F. Hwang, Properly embedded and immersed minimal surfaces in the Heisenberg group, Bull. Austral. Math. Soc. 70 (2004), 507-520.

[CK] P. Collin and R. KRUST, Le problème de Dirichlet pour l'équation des surfaces minimales sur des domaines non bornès, Bull. Soc. Math. France 119 (1991), 443-462.

[DGN] D. Danielli, N. Garofalo and D-M. Nhieu, Minimal surfaces, surfaces of constant mean curvature and isoperimetry in Carnot groups, Preprint, 2001.

[FS] G. B. Folland and E. M. STEIN, Estimates for the $\bar{\partial}_{b}$-complex and analysis on the Heisenberg group, Comm. Pure Appl. Math. 27 (1974), 429-522.

[FSS] B. Franchi, R. SERAPIONi and F. Serra Cassano, Rectifiability and perimeter in the Heisenberg group, Math. Ann. 321 (2001), 479-531.

[GN] N. Garofalo and D.-M. Nhieu, Isoperimetric and Sobolev inequalities for CarnotCaratheodory spaces and the existence of minimal surfaces, Comm. Pure Appl. Math. 49 (1996), 1081-1144.

[GP1] N. GaRofalo and S. Pauls, The Bernstein problem in the Heisenberg group, arXiv: math. DG/0209065.

[GP2] N. GaRofalo and S. PaUls, The Bernstein problem in the Heisenberg group, preprint, 2004.

[HL] R. Harvey and H. B. Lawson, Calibrated geometries, Acta Math. 148 (1982), 47-157.

[Hw1] J. F. Hwang, Comparison principles and Liouville theorems for prescribed mean curvature equation in unbounded domains, Ann. Scuola Norm. Sup. Pisa Cl. Sci. 15 (1988), 341-355.

[Hw2] J. F. HwAng, Structural inequalities method for uniqueness theorems for the minimal surface equation, Proc. of CMA (Joint Australia-Taiwan Workshop on Analysis and Application, Brisbane, March 1997), Australian National University, Vol. 37, 1999, 4752.

[JL1] D. JERISON and J. M. LEE, The Yamabe problem on CR manifolds, J. Differential Geom. 25 (1987), 167-197.

[JL2] D. JERISON and J. M. LEE, Intrinsic CR normal coordinates and the CR Yamabe problem, J. Differential Geom. 29 (1989), 303-343. 
[Jo] F. John, "Partial Differential Equations", Springer-Verlag, 4th ed., 1982.

[La] H. B. LAwson JR., Complete minimal surfaces in $S^{3}$, Ann. of Math. 92 (1970), 335-374.

[Lee] J. M. LEE, The Fefferman metric and pseudohermitian invariants, Trans. Amer. Math. Soc. 296 (1986), 411-429.

[LM] G. LeONARDI and S. MASNOU, On the isoperimetric problem in the Heisenberg group $H^{n}$, to appear on Annali Mat. Pura e Appl., 2002.

[LR] G. LEONARDI and S. RigOt, Isoperimetric sets on Carnot groups, Houston J. Math. (2003).

[Mik] V. M. Miklyukov, On a new approach to Bernstein's theorem and related questions for equations of minimal surface type, Mat. Sb. 108 (150) (1979), 268-289; English transl. in Math. USSR Sb. 36 (1980), 251-271.

[Mil] J. Milnor, “Topology from the Differentiable Viewpoint”, University of Virginia Press, 1965 .

[Mo] G. Monge, "Application de l'Analyse à la Géométrie", Paris, Bachelier, 1850.

[Os] R. Osserman, “A Survey of Minimal Surfaces”, Dover Publications, Inc., New York, 1986.

[Pan] P. PAnsu, Une inegalite isoperimetrique sur le groupe de Heisenberg, C.R. Acad. Sci. Paris I 295 (1982), 127-130.

[Pau] S. D. Pauls, Minimal surfaces in the Heisenberg group, Geom. Dedicata 104 (2004), 201-231.

[Sp] M. SpIVAK, "A Comprehensive Introduction to Differential Geometry”, Vol. 3, Publish or Perish Inc., Boston, 1975.

[St] S. Sternberg, "Lectures on Differential Geometry”, 2nd ed., Chelsea Publishing Company, New York, 1983.

[SY] R. SchOEN and S.-T. YAu, On the proof of the positive mass conjecture in general relativity, Comm. Math. Phys. 65 (1979), 45-76.

[Ta] N. TANAKA, "A Differential Geometric Study on Strongly Pseudo-Convex Manifolds", Kinokuniya Co. Ltd., Tokyo, 1975.

[We] S. M. WEBSTER, Pseudohermitian structures on a real hypersurface, J. Differential Geom. 13 (1978), 25-41.

Institute of Mathematics

Academia Sinica

Nankang, Taipei, Taiwan, 11529, R.O.C.

cheng@math.sinica.edu.tw

Institute of Mathematics

Academia Sinica

Nankang, Taipei, Taiwan, 11529, R.O.C.

majfh@math.sinica.edu.tw

SISSA

Via Beirut 2-4

34014 Trieste, Italy

malchiod@sissa.it

Department of Mathematics

Princeton University

Princeton, NJ 08544, U.S.A.

yang@Math.Princeton.EDU 